\title{
WestVirginiaUniversity
}

THE RESEARCH REPOSITORY @ WVU

Graduate Theses, Dissertations, and Problem Reports

2022

\section{On Paradoxical Examples of Real Functions}

Cheng-Han Pan

West Virginia University, chpan@mix.wvu.edu

Follow this and additional works at: https://researchrepository.wvu.edu/etd

\section{Recommended Citation}

Pan, Cheng-Han, "On Paradoxical Examples of Real Functions" (2022). Graduate Theses, Dissertations, and Problem Reports. 10300.

https://researchrepository.wvu.edu/etd/10300

This Dissertation is protected by copyright and/or related rights. It has been brought to you by the The Research Repository @ WVU with permission from the rights-holder(s). You are free to use this Dissertation in any way that is permitted by the copyright and related rights legislation that applies to your use. For other uses you must obtain permission from the rights-holder(s) directly, unless additional rights are indicated by a Creative Commons license in the record and/ or on the work itself. This Dissertation has been accepted for inclusion in WVU Graduate Theses, Dissertations, and Problem Reports collection by an authorized administrator of The Research Repository @ WVU.

For more information, please contact researchrepository@mail.wvu.edu. 


\title{
ON PARADOXICAL EXAMPLES OF REAL FUNCTIONS
}

by

\section{Cheng-Han Pan}

\author{
Dissertation submitted to the \\ Eberly College of Arts and Sciences \\ at West Virginia University \\ in partial fulfillment of the requirements for the degree of \\ Philosophiae Doctor \\ in \\ Mathematica
}

Krzysztof Chris Ciesielski, Ph.D., Committee Chairperson

Paul Gartside, Ph.D.

Dening Li, Ph.D.

Adrian Tudorascu, Ph.D.

Jerzy Wojciechowski, Ph.D.

Department of Mathematics

Morgantown, West Virginia

2021

Keywords: Sierpiński-Zygmund function, Darboux-like function, Pompeiu derivative,

Pompeiu-like function, Nowhere-monotone differentiable function, Jordan decomposition

Copyright (C) 2021 Cheng-Han Pan 


\title{
ABSTRACT
}

\section{On Paradoxical Examples of Real Functions}

\author{
by Cheng-Han Pan
}

This dissertation is a summary of the author's research work, supervised by Professor Krzysztof Ciesielski, based on three published articles in the Journal of Mathematical Analysis and Applications and one published article in the Banach Journal of Mathematical Analysis. Our work focuses on the study of paradoxical real functions regarding differentiability and generalized continuity within the foundations of real analysis. The reasons why they are paradoxical are directly connected to their definitions, which will be provided and explained in the later texts. Note that all functions discussed in the dissertation are single-variable and real valued, that is, well-defined from a proper or improper subset of the set $\mathbb{R}$ of real numbers into $\mathbb{R}$.

The material is presented in two independent chapters. Chapter 1 consists of a study of nowheremonotone differentiable functions to which we refer as the differentiable monsters. Almost 130 years after A. Köpcke constructed the first differentiable monster and after its many simplifications, K. Ciesielski noticed a few years ago the simplest such construction by far. This construction was a shifted difference of two arbitrary strictly increasing Pompeiu-like functions, that is, of differentiable functions with their derivatives vanishing on a dense subset of their domain. However, not every differentiable monster of bounded variation admits such a Jordan-like decomposition that possesses Pompeiu-likeness. We have first characterized differentiable monsters that can be decomposed in such a "nice" way as those that are a difference of two increasing differentiable functions. Secondly, as Jarník's extension theorem allows a differentiable extension to be as "good" as being smooth on the extended parts, we work on the other direction and make a differentiable extension as "bad" as being nowhere-monotone on the extended parts. Since it is an easy consequence of Darboux's theorem that a differentiable monster must be Pompeiu-like, we have shown that a typical function in a designated complete metric space, which consists of all differentiable extensions that are Pompeiulike on the extended part, is nowhere-monotone on the extended part. On the other hand, we have also shown that the family of nowhere nowhere-monotone functions is dense in this space.

In Chapter 2, we additionally impose a set-theoretical axiom that the set $\mathbb{R}$ is not a union of less than continuum-many meager sets. A Darboux function is a function that satisfies the intermediate value property, so the classes of Darboux-like functions represent a group of functions that are continuous in a generalized sense. On the contrary, Sierpiński-Zygmund functions, first constructed in 1923 by W. Sierpiński and A. Zygmund, have as little of the standard continuity as possible. The algebra of subsets generated by these classes and the Sierpiński-Zygmund functions has nine atoms, that is, the smallest nonempty elements of the algebra. In this work, we have crafted a lemma that easily create examples in each of these nine atoms with transfinite induction. Note that the examples within the seven of the nine atoms were first discovered by K. Ciesielski and C.-H. Pan and had been included in a survey of Sierpiński-Zygmund functions in 2019 by K. Ciesielski and J. Seoane-Sepúlveda. As lineability of the main classes of Darboux-like functions, as well as of Sierpiński-Zygmund functions, has been intensively studied, our presented work has caused some on-going researches in the lineability of the nine smaller classes mentioned above. 
To Wen Wang

For her encouragement and her faith,

Because she always understood... 


\section{Acknowledgments}

Firstly, I would like to express my deepest and most sincere gratitude to my advisor Professor Krzysztof Ciesielski. His guidance not only strengthened my skills in mathematics, but also improved my scientific writing style. I appreciate his immeasurable patience in preparing me to be an independent researcher.

I would also like to thank my committee members, Professor Krzysztof Ciesielski, Professor Paul Gartside, Professor Dening Li, Professor Adrian Tudorascu and Professor Jerzy Wojciechowski for their expertise and insightful feedback in preparing this dissertation.

Furthermore, I would like to acknowledge the Mathematics department at West Virginia University, along with Professor Krzysztof Ciesielski, Professor Harvey Diamond, Professor Edgar Fuller, Professor Harry Gingold, Professor Harumi Hattori, Professor Hong-Jian Lai, Professor Dening Li, Professor Kevin Milans, Professor Casian Pantea, Professor Adrian Tudorascu, Professor Murali Vemuri and Professor Jerzy Wojciechowski who taught me various advanced mathematics courses throughout my graduate studies.

Additionally, I would like to express my appreciation to Professor Krista Bresock, Geoffery Cooke, Richard Devine, Doctor Keith Gallagher, Jose Jimenez, Habibolla Latifizadeh, Enni Le, Chong Li, Doctor Lucian Mazza, Professor David Miller, Wesley Neill and Nurul Wahyuni who offered many meaningful academic discussions and encouragement over the years. My appreciation goes to David Macaulay, Avery Mascorro, Doctor Lucian Mazza and Lavina Wilson as well for their occasional help in English, and to Rebecca Alexander for her help writing this acknowledgment.

Finally, I would like to thank my parents for supporting my decision to pursue my degree abroad. This opportunity has expanded my thinking and enriched my life experience. 


\section{Contents}

$\begin{array}{ll}\text { Abstract } & \text { ii }\end{array}$

Acknowledgments $\quad$ iv

List of Figures $\quad$ vii

Notations $\quad$ viii

1 Paradoxes in Differentiability 1

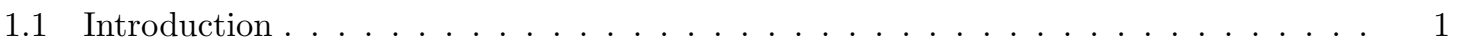

1.2 A simple construction of differentiable monsters . . . . . . . . . . . . . . . . . 2

1.3 Differentiable monsters and a Jordan-like decomposition . . . . . . . . . . . . . . 4

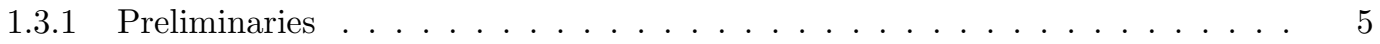

1.3.2 A Jordan-like decomposability of Pompeiu-like functions . . . . . . . . . . . 7

1.3.3 Examples and discussion . . . . . . . . . . . . . . . . . . . . . 12

1.3.4 Related open problems . . . . . . . . . . . . . . . . . . . . . . . . . 18

1.4 Monstrous Extentions . . . . . . . . . . . . . . . . . . . . . . . . . . . 19

1.4.1 Differentiable monsters extending $\mathfrak{f} \ldots \ldots \ldots \ldots \ldots \ldots$

1.4.2 Weierstrass's monsters extending $\mathfrak{f} \ldots \ldots \ldots \ldots 22$

1.5 Typicality of differentiable extensions in $\mathrm{E}_{\Phi}(\mathbb{R}) \ldots \ldots \ldots \ldots \ldots \ldots$

1.5.1 The space of differentiable extensions . . . . . . . . . . . . . . 23

1.5.2 Differentiable extensions that are nowhere-monotone . . . . . . . . . . . 25

1.5.3 Differentiable extensions that are nowhere nowhere-monotone . . . . . . . 26

2 Paradoxes in Continuity 29

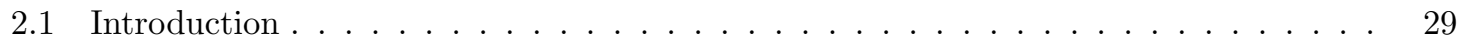

2.2 Examples of Sierpiński-Zygmund functions in the class of Darboux-like functions . . 33 


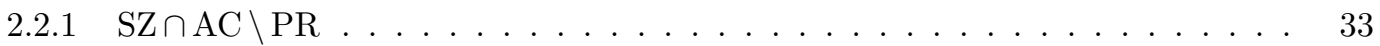

$2.2 .2 \quad \mathrm{SZ} \cap \mathcal{D} \backslash(\mathrm{PR} \cup \mathrm{Conn}) \ldots \ldots \ldots \ldots \ldots \ldots \ldots$

$2.2 .3 \quad \mathrm{SZ} \cap \mathcal{D} \cap \mathrm{CIVP} \backslash \mathrm{Conn} \ldots \ldots \ldots \ldots \ldots \ldots \ldots$

$2.2 .4 \quad \mathrm{SZ} \cap \mathcal{D} \cap \mathrm{PR} \backslash(\mathrm{Conn} \cup \mathrm{CIVP}) \ldots \ldots \ldots \ldots \ldots \ldots$

$2.2 .5 \quad \mathrm{SZ} \cap \mathrm{Conn} \cap \mathrm{CIVP} \backslash \mathrm{AC} \ldots \ldots \ldots \ldots \ldots \ldots \ldots$

$2.2 .6 \quad \mathrm{SZ} \cap \mathrm{Conn} \cap \mathrm{PR} \backslash(\mathrm{AC} \cup \mathrm{CIVP}) \ldots \ldots \ldots \ldots \ldots \ldots$

$2.2 .7 \quad \mathrm{SZ} \cap \mathrm{Conn} \backslash(\mathrm{AC} \cup \mathrm{PR}) \ldots \ldots \ldots \ldots \ldots \ldots$

2.2.8 $\mathrm{SZ} \cap \mathrm{AC} \cap \mathrm{PR} \backslash \mathrm{CIVP}$ and $\mathrm{SZ} \cap \mathrm{AC} \cap \mathrm{CIVP} \ldots \ldots \ldots \ldots \ldots$

$\begin{array}{ll}\text { Bibliography } & 47\end{array}$

Index $\quad 53$ 


\section{List of Figures}

1.1 The inclusions, indicated by $\Rightarrow$ when strictness is unknown and by $\rightarrow$ when they are known to be strict, between the indicated classes. The indicated Examples justify strictness of all vertical inclusions between the associated rows. Similarly, the labels above the top horizontal arrows justify strictness of all horizontal inclusions between the associated columns. . . . . . . . . . . . . . . . . . . . 14

1.2 An illustration of $h_{n}$ on $\left[p_{n+1}, p_{n}\right]$ used in the construction of $\gamma$ in Example 1.3.12. . 16

2.1 All inclusions, indicated by arrows, among the Darboux-like classes of functions from $\mathbb{R}$ to $\mathbb{R}$. The only inclusions among the intersections of these classes are those that follows trivially from this schema. (See $[35],[19]$, or $[27]$.) $\ldots \ldots \ldots \ldots$

2.2 Six Darboux-like classes of functions that consistently contain SZ-functions. Arrows indicate strict inclusions. . . . . . . . . . . . . . . . . . . . . . .

2.3 Illustration for the proof of Lemma 2.2.18. Left figure corresponds to the case when $[p, x) \subseteq \pi[H \cap([p, x) \times(-\infty, y])]$. The right figure addresses the case leading to the

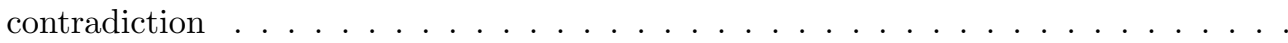




\title{
Notations
}

\author{
ZFC Zermelo-Fraenkel axioms \\ $\emptyset \quad$ Empty set \\ $x \in X \quad x$ is an element of $X$ \\ $X \subseteq Y, X \supseteq Y \quad X$ is a subset/superset of $Y$ \\ $X \cup Y, X \cap Y \quad$ The union/intersection of sets $X$ and $Y$ \\ $X \backslash Y \quad$ The difference of sets $X$ and $Y$ \\ $\operatorname{cl}(X) \quad$ The closure of a set $X$ \\ $X^{\prime} \quad$ The collection of accumulation points of a set $X$ \\ $|X| \quad$ The cardinality of a set $X$ \\ $\mathbb{N} \quad$ The set of natural numbers \\ $\mathbb{Z} \quad$ The set of integers \\ $\mathbb{Q} \quad$ The set of rational numbers \\ $\mathbb{R} \quad$ The set of real numbers \\ $\omega \quad$ The cardinality of $\mathbb{N}$ \\ $\mathfrak{c} \quad$ The cardinality of $\mathbb{R}$ \\ $\operatorname{cov}_{\mathrm{M}} \quad$ The covering number of $\mathbb{R}$ with meager sets \\ $Y^{X} \quad$ The family of functions from a set $X$ into a set $Y$ \\ $f: X \rightarrow Y \quad$ A function from a set $X$ into a set $Y$ \\ $\operatorname{dom}(f) \quad$ The domain of a function $f$ \\ $f \uparrow_{A} \quad$ The restriction of a function $f$ on a set $A$ \\ $f[A] \quad$ The image of a set $A$ with respect to a function $f$ \\ $f^{-1}(B) \quad$ The preimage of a set $B$ with respect to a function $f$ \\ $[f=a],[f>a],[f \geq a] \quad$ The preimage of a set $\{a\} /(a, \infty) /[a, \infty)$ with respect to a function $f$ \\ $\|f\|_{\infty} \quad$ The infinity norm of a function $f$ \\ $\|f\|_{\mathrm{C}^{1}} \quad$ The $\mathrm{C}^{1}$-norm of a function $f$ \\ $\mathrm{TV}_{f}, \mathrm{PV}_{f}, \mathrm{NV}_{f} \quad$ The total/positive/negative variation function of a function $f$ \\ $f^{\prime} \quad$ The first derivative of a function $f$ \\ $\mathrm{D}^{+} f, \mathrm{D}^{-} f, \mathrm{D}_{+} f, \mathrm{D}_{-} f \quad$ The upper-right/left and lower-right/left Dini derivative of a function $f$
}




\section{Chapter 1}

\section{Paradoxes in Differentiability}

This chapter is based on the three published articles [21,22,65], in which [21] started the research of finding "bad" extensions of differentiable functions, [22] studied its typicality in a designated complete metric space of differentiable extensions, and [65] refined the extension method and applied it to modify differentiable functions of bounded variation. ${ }^{1}$ The presentation of this chapter starts with the results from the most recent article [65] and then revisits the earlier results from $[21,22]$.

\section{$1.1 \quad$ Introduction}

How much continuity follows from differentiability? It is well-known that the answer is all. On the other hand, how much differentiability follows from continuity? In the past, mathematicians commonly believed that a continuous function should be differentiable on a "significantly large" subset of its domain. Shortly, the first published example of a Weierstrass's monster - everywhere continuous but differentiable at no point - was given by K. Weierstrass and appeared in an 1872 paper. $^{2}$ Since then, many different examples of Weierstrass's monsters have been described. ${ }^{3}$ One of the most elegant among them, in our opinion, is the Takagi-van der Waerden construction. ${ }^{4}$ Our

\footnotetext{
${ }^{1}$ Authors publishing in Elsevier journals have rights to use their works, in full or in part, for a wide range of scholarly, non-commercial purposes, which include inclusion in a thesis or dissertation, without needing to seek permission as long as DOI links to the versions of records on ScienceDirect are provided.

${ }^{2}$ See [31] or [78]. It is worthy to mention that both C. Cellérier [14] and B. Bolzano [41,44] described such functions before K. Weierstrass. However, their constructions were not published in their lifetimes and were rediscovered considerably after 1872 .

${ }^{3}$ See [75] or a book [40].

${ }^{4}$ Takagi-van der Waerden functions used to be called just van der Waerden functions, based on van der Waerden's influential 1930 paper [77]. Since then, it was noticed that T. Takagi had already described such functions in his 1901 paper [74]. However, this work was overlooked for a time in the West due to Japan's isolation in the early twentieth century. See a survey [2] of the Takagi functions.
} 
favorite Takagi-van der Waerden type of a Weierstrass's monster is defined, on $\mathbb{R}$, as

$$
f(x):=\sum_{n=0}^{\infty} 4^{n} f_{n}(x)
$$

where $f_{n}(x):=\min _{k \in \mathbb{Z}}\left|x-\frac{k}{8^{n}}\right|$ is the distance from $x \in \mathbb{R}$ to the set $\frac{1}{8^{n}} \mathbb{Z}=\left\{\frac{k}{8^{n}}: k \in \mathbb{Z}\right\} .{ }^{5}$ Notice that every Weierstrass's monster is nowhere-monotone. ${ }^{6}$ Seemingly, nowhere-monotone is a "bad" property for differentiability due to the fractal nature of spikes that it has. Thus, it is natural to inquire on how much differentiability a nowhere-monotone function can have. Surprisingly, the answer to this question is all. The existence of everywhere differentiable nowhere-monotone functions, to which we refer as differentiable monsters, is considerably less known in the mathematical community than that of the Weierstrass's monsters.

The subject of Chapter 1 is a study in different variations of differentiable monsters and their constructions. In particular, in Section 1.2 we present a new simple construction of a differentiable monster as a difference of two monotone differentiable functions, which has inspired us to discuss, in Section 1.3, a Jordan-like decomposability problem for differentiable monsters. In Sections 1.4 and 1.5, we take advantage of Jarník's extension theorem together with Lemma 1.3.8 from Section 1.3 to study the differentiable extensions and their spaces.

\subsection{A simple construction of differentiable monsters}

Definition 1.2.1. A function is called a differentiable monster provided it maps a nontrivial interval $J \subseteq \mathbb{R}$ into $\mathbb{R}$, it is differentiable, and monotone on no nontrivial subinterval of $J$.

What is so fascinating about a differentiable monster? It must be the fact that its graph simultaneously has two seemingly contradictive properties of being "silky" and very "coarse". The first such example was constructed in late 1880s by Alfred Köpcke in three consecutive papers [52-54]. Since then, a multitude of different constructions of such maps has been described, see $[8,17,30,48,79,80]$. For more history on the subject, see [10, section 2]. It is an easy consequence of the mean value theorem that a differentiable $f: J \rightarrow \mathbb{R}$ is a differentiable monster if, and only if,

the sets $\left[f^{\prime}>0\right]$ and $\left[f^{\prime}<0\right]$ are dense in $J$.

\footnotetext{
${ }^{5}$ See [17] or [71, theorem 7.18].

${ }^{6}$ Suppose $f$ is monotone on any nontrivial $(a, b) \subseteq \mathbb{R}$. By Lebesgue differentiation theorem, $f$ is differentiable almost everywhere, in sense of Lebesgue measure, in $(a, b)$.
} 
Here, for any $g: X \rightarrow \mathbb{R}$ and $M \in \mathbb{R}$, the symbols $[g>M]$ and $[g<M]$ denote the preimages $g^{-1}((M, \infty))$ and $g^{-1}((-\infty, M))$. The sets $[g \geq M],[g \leq M]$, and $[g=M]$ are defined similarly. Since any derivative has the Darboux property, ${ }^{7}$ the property (1.2) implies that for every differentiable monster $f: J \rightarrow \mathbb{R}$,

$$
\left[f^{\prime}=0\right] \text { is dense in } J
$$

Köpcke's construction of a differentiable monster is rather complicated. To better understand it, Dimitrie Pompeiu in his 1905 Ph.D. thesis [67], written under supervision of Henri Poincaré, studied the functions that have the property (1.3). Nowadays, a derivative satisfying (1.3)-vanishing on a dense subset of its domain - is called a Pompeiu derivative. ${ }^{8}$ In Chapter 1, we are interested in the primitives of Pompeiu derivatives, to which we refer as Pompeiu-like functions. ${ }^{9}$

Definition 1.2.2. A function $f$ from a nontrivial interval $J \subseteq \mathbb{R}$ into $\mathbb{R}$ is called Pompeiu-like provided it is differentiable and satisfies (1.3), that is, its derivative vanishes on a dense subset of its domain.

Pompeiu, in his 1907 article [68], described a very simple construction of a strictly increasing Pompeiu-like function $G$ from a bounded interval $\left(t^{\prime}, t^{\prime \prime}\right) \subseteq \mathbb{R}$ onto $\left(x^{\prime}, x^{\prime \prime}\right)$ : it is the inverse of a map $F:\left(x^{\prime}, x^{\prime \prime}\right) \rightarrow\left(t^{\prime}, t^{\prime \prime}\right)$ defined as

$$
F(x)=\sum_{n \in \mathbb{N}} A_{n}\left(x-q_{n}\right)^{\frac{1}{3}},
$$

where $\left\{q_{n}: n \in \mathbb{N}\right\}$ is any countable dense subset of $\left(x^{\prime}, x^{\prime \prime}\right)$, and $\left\langle A_{n}\right\rangle_{n \in \mathbb{N}}$ is any sequence of positive numbers such that $\sum_{n \in \mathbb{N}} A_{n}$ converges. Note that $F$ is strictly increasing and therefore invertible, as is each map $A_{n}\left(x-q_{n}\right)^{\frac{1}{3}}$. Also, $F$ admits at every point a positive (finite or infinite) derivative, infinite at each $q_{n}$, so its inverse function $G$ is differentiable, admitting at every point a non-vertical tangent, with zero derivative at each $F\left(q_{n}\right)$.

It looked that this simply defined functions should be easily turned into a differentiable monster. Nevertheless, it took over a century to transform $G$ by the elementary means into a differentiable monster. Specifically, it was known for a while (see [30] or [10, section 2]) that if $G:\left(t^{\prime}, t^{\prime \prime}\right) \rightarrow\left(x^{\prime}, x^{\prime \prime}\right)$ is a strictly increasing Pompeiu-like function, then there exists a diffeomorphism $\delta:\left(t^{\prime}, t^{\prime \prime}\right) \rightarrow\left(t^{\prime}, t^{\prime \prime}\right)$ such that $f:=(G \circ \delta)-G$ is a differentiable monster. However, the construction of $\delta$ still required

\footnotetext{
${ }^{7}$ It is commonly known as the intermediate value property. See [26, theorem 2.1] or [3, theorem 5.16].

${ }^{8}$ It is not clear who started this trend, but D. Pompeiu himself called those derivatives fonctions de M. Köpcke in [67] and [68].

${ }^{9}$ This is to clarify the slight difference between Pompeiu functions and Pompeiu-like functions. In a 1963 article of Solomon Marcus [56], he defined a $G:[a, b] \rightarrow \mathbb{R}$ to be a Pompeiu function provided $G^{\prime}$ is bounded, and $\left[G^{\prime}=0\right]$ "equals" a dense set without interior. Marcus's definition does not quite fit this article since we have not only managed to handle cases of unbounded derivatives, but also like to have a Banach space when derivatives are bounded. Therefore, we remove "boundedness" and replace "equals" with "contains".
} 
some work. This changed with a publication of K. Ciesielski's 2018 article [17], where it is noticed that if $h: \mathbb{R} \rightarrow \mathbb{R}$ is strictly increasing Pompeiu-like, then for any $t$ in a dense $G_{\delta}$ subset of $\mathbb{R}$,

$$
\text { the map } f(x):=h(x-t)-h(x) \text { is a differentiable monster }
$$

as the choice of $t$ particularly makes sets $\left[h^{\prime}(x-t)>0\right] \cap\left[h^{\prime}=0\right]$ and $\left[h^{\prime}(x-t)=0\right] \cap\left[h^{\prime}>0\right]$ both dense in $\mathbb{R}$.

\subsection{Differentiable monsters and a Jordan-like decomposition}

The above construction (1.5) has led us to examine two questions:

(Q1) Is every differentiable monster a difference of two strictly increasing Pompeiu-like functions?

(Q2) If not, is there a nice characterization of differentiable monsters that have such representations?

In Example 1.3.14, we constructed a differentiable monster which is not of bounded variation. In particular, such a function is not a difference of two monotone functions. ${ }^{10}$ On the other hand, we proved that (Q1) has a positive answer on a large subclass of the class of all differentiable monsters:

Theorem 1.3.1. Let $f: J \rightarrow \mathbb{R}$ be a Pompeiu-like function. If $f^{\prime}$ is bounded either from above or from below, then $f$ is a difference of two (strictly) increasing Pompeiu-like functions.

In spite of Example 1.3.14, Theorem 1.3.1 implies, in Corollary 1.3.10, that (Q1) has an "almost" positive answer in a sense that for every Pompeiu-like function $f$ and therefore every differentiable monster, there are many nontrivial intervals $I$ such that $f \uparrow_{I}$ is a difference of two increasing Pompeiu-like functions. Thus, one cannot construct a differentiable monster without having at least a piece being a difference of two increasing Pompeiu-like functions.

From Theorem 1.3.1, we have also deduced the following characterization that constitutes an answer to (Q2):

Corollary 1.3.2. Let $f: J \rightarrow \mathbb{R}$ be a Pompeiu-like function. Then $f$ is a difference of two increasing Pompeiu-like functions if, and only if $f$ is a difference of two increasing differentiable functions.

Finally, Section 1.3 is organized in the following order. In Section 1.3.1, we present several simple and well-known propositions that are used in Section 1.3.2, which contains all the technical proofs toward the main results. In Section 1.3.3, we include a remark and three examples that drew a

\footnotetext{
${ }^{10}$ See Jordan's decomposition [46], it gives a negative answer to (Q1).
} 
picture of the inclusive relations among some subclasses of Pompeiu-like functions. In Section 1.3.4, we raise some interesting unsolved problems in classical real analysis.

\subsubsection{Preliminaries}

Certainly, the family of Pompeiu-like functions has some nice properties inherited from the family of Pompeiu derivatives. The first three propositions constitute simple and/or folklore results on Pompeiu-like functions.

Proposition 1.3.3. If $f$ is a Pompeiu-like function defined on an interval $J$ and $M>0$, then $\left[f^{\prime} \geq M\right]$ is nowhere-dense in $J$.

Proof. It is well-known that $f^{\prime}$ is of Baire class one, ${ }^{11}$ that is, the preimage of any open set under $f^{\prime}$ is an $F_{\sigma}$ set. In other words, $\left[f^{\prime}=0\right]$ and $\left[f^{\prime} \geq M\right]$ are both $G_{\delta}$ sets in $J$. With $\left[f^{\prime}=0\right]$ being dense in $J,\left[f^{\prime} \geq M\right]$ cannot be dense in any subinterval as, by Baire category theorem, this would imply $\left[f^{\prime}=0\right] \cap\left[f^{\prime} \geq M\right] \neq \emptyset$, a contradiction.

Proposition 1.3.4. Let $J \subseteq \mathbb{R}$ be any nontrivial interval.

(i) The family $\mathcal{P}(J)$ of all Pompeiu-like functions on $J$ forms a linear space over $\mathbb{R}$.

(ii) The family $\mathcal{P}_{\mathrm{b}}(J)$ of all $f \in \mathcal{P}(J)$ with

$$
\|f\|_{\mathrm{C}^{1}}:=\|f\|_{\infty}+\left\|f^{\prime}\right\|_{\infty}<\infty
$$

forms a Banach space over $\mathbb{R}$ with the $\mathrm{C}^{1}$-norm $\|\cdot\|_{\mathrm{C}^{1}}$.

Proof. (i) Clearly, any linear combination $a f+b g$ of $f, g \in \mathcal{P}(J)$ is differentiable. It belongs to $\mathcal{P}(J)$ since $\left[(a f+b g)^{\prime}=0\right]$ contains a dense $G_{\delta}$ set $\left[f^{\prime}=0\right] \cap\left[g^{\prime}=0\right]$.

(ii) If $f, g \in \mathcal{P}_{\mathrm{b}}(J)$, then $\|a f+b g\|_{\mathrm{C}^{1}} \leq|a|\|f\|_{\mathrm{C}^{1}}+|b|\|g\|_{\mathrm{C}^{1}}<\infty$. So, by (i), $a f+b g \in \mathcal{P}_{\mathrm{b}}(J)$. If a sequence $\left\langle f_{n}\right\rangle_{n \in \mathbb{N}}$ in $\mathcal{P}_{\mathrm{b}}(J)$ is Cauchy with respect to $\|\cdot\|_{\mathrm{C}^{1}}$, then by a well-known result, ${ }^{12}$ its limit $f$ is well defined, differentiable, and has a bounded $\mathrm{C}^{1}$-norm. Lastly, $f$ belongs to $\mathcal{P}_{\mathrm{b}}(J)$ since $\left[f^{\prime}=0\right]$ contains a dense $G_{\delta}$ set $\bigcap_{n \in \mathbb{N}}\left[f_{n}^{\prime}=0\right]$.

In what follows, we often use the following terminology.

\footnotetext{
${ }^{11}$ See [26, theorem 2.2].

${ }^{12}$ See [76, theorem 9.37] or [3, theorem 9.13].
} 
Definition 1.3.5. A Pompeiu-like function $\xi:[0,1] \rightarrow \mathbb{R}$ is said to be a $P$-filling provided

$$
\left\|\xi^{\prime}\right\|_{\infty}<\infty, \xi^{\prime}(0)=\xi^{\prime}(1)=0=\xi(0), \text { and } \xi(1)=1
$$

The following proposition establishes the existence of P-fillings with some additional properties.

Proposition 1.3.6. There exists a pair of strictly increasing P-fillings $\varphi$ and $\psi$ such that both sets $\left[\varphi^{\prime}>0\right] \cap\left[\psi^{\prime}=0\right]$ and $\left[\varphi^{\prime}=0\right] \cap\left[\psi^{\prime}>0\right]$ are dense in $[0,1]$. In particular, a $\varphi-b \psi$ is nowhere-monotone for every $a, b>0$, and $\sigma=2 \varphi-\psi$ is a nowhere-monotone $P$-filling.

Proof. Take a differentiable monster $f(x):=h(x-t)-h(x)$ from (1.5) where $h:=G \circ \delta$ is a strictly increasing Pompeiu-like function with $G:\left(t^{\prime}, t^{\prime \prime}\right) \rightarrow\left(x^{\prime}, x^{\prime \prime}\right)$ being the inverse of $F$ defined in (1.4) and $\delta: \mathbb{R} \rightarrow\left(t^{\prime}, t^{\prime \prime}\right)$ being an increasing diffeomorphism of bounded derivative. Notice also that $h$ has a bounded derivative as $F^{\prime}$ is bounded away from zero. We write $h_{t}(x):=h(x-t)$ and choose $c<d$ from a dense $G_{\delta}$ set $\left[h_{t}^{\prime}=0\right] \cap\left[h^{\prime}=0\right]$ and let $\mathrm{L}:[0,1] \rightarrow[c, d]$ be an increasing linear surjection. Then

$$
\varphi(x):=\frac{\left(h_{t} \circ \mathrm{L}\right)(x)-h_{t}(c)}{h_{t}(d)-h_{t}(c)} \quad \text { and } \quad \psi(x):=\frac{(h \circ \mathrm{L})(x)-h(c)}{h(d)-h(c)}
$$

are a pair of strictly increasing P-fillings such that $\left[\varphi^{\prime}>0\right] \cap\left[\psi^{\prime}=0\right]$ and $\left[\varphi^{\prime}=0\right] \cap\left[\psi^{\prime}>0\right]$ are both dense in $[0,1]$. Consequently, $a \varphi-b \psi$ is nowhere-monotone for every $a, b>0$ since $\left[(a \varphi-b \psi)^{\prime}>0\right]$ and $\left[(a \varphi-b \psi)^{\prime}<0\right]$ are both dense in $[0,1]$ as they contain $\left[\varphi^{\prime}>0\right] \cap\left[\psi^{\prime}=0\right]$ and $\left[\varphi^{\prime}=0\right] \cap\left[\psi^{\prime}>0\right]$ respectively. Clearly, $\sigma=2 \varphi-\psi$ is a nowhere-monotone P-filling.

Proposition 1.3.7. Let $J \subseteq \mathbb{R}$ be any nontrivial interval, $P \subseteq J$ be closed, and $\varepsilon>0$.

(i) There exists a smooth map $H_{P}: J \rightarrow \mathbb{R}$ such that

$$
H_{P}=H_{P}^{\prime}=0 \text { on } P, H_{P}>0 \text { on } J \backslash P \text {, and }\left\|H_{P}\right\|_{\infty} \leq \varepsilon \text {. }
$$

(ii) If $f, g: J \rightarrow \mathbb{R}$ and $f$ is differentiable, then

$$
|g-f| \leq H_{P} \text { on } J \text { implies } g^{\prime} \text { exists on } P \text { and } g^{\prime} \uparrow_{P}=f^{\prime} \uparrow_{P} \text {. }
$$

Proof. By [55, proposition 2.25], for every $n \in \mathbb{N}$ there is a $C^{\infty}$ map $g_{n}: \mathbb{R} \rightarrow[0,1]$ such that $g_{n}=1$ on $\left\{x \in \mathbb{R}: \operatorname{dist}(x, P) \geq \frac{1}{n+1}\right\}$ and $g_{n}=0$ on $\left\{x \in \mathbb{R}: \operatorname{dist}(x, P) \leq \frac{1}{n+2}\right\}$. Then $H_{P}:=$ $\varepsilon \sum_{n \in \mathbb{N}} c_{n} g_{n} \Gamma_{J}$ is as needed, provided $c_{n} g_{n}^{(i)}[\mathbb{R}] \subseteq\left[0,2^{-n}\right]$ for all $i \leq n$. 
(ii) is an application of the squeeze theorem on the boundary of $P$. It is enough to show that $\lim _{h \rightarrow 0} \frac{g(x+h)-g(x)}{h}=f^{\prime}(x)$ for every $x \in P$. Indeed, if $Q(x, h)=\frac{g(x+h)-g(x)}{h}-\frac{f(x+h)-f(x)}{h}$, then $\lim _{h \rightarrow 0} Q(x, h)=0$ since $0 \leq|Q(x, h)|=\left|\frac{g(x+h)-f(x+h)}{h}\right| \leq\left|\frac{H_{P}(x+h)-H_{p}(x)}{h}\right| \stackrel{h \rightarrow 0}{\longrightarrow} H_{P}^{\prime}(x)=0$. Therefore, $\lim _{h \rightarrow 0} \frac{g(x+h)-g(x)}{h}=\lim _{h \rightarrow 0} Q(x, h)+\lim _{h \rightarrow 0} \frac{f(x+h)-f(x)}{h}=f^{\prime}(x)$, as needed.

\subsubsection{A Jordan-like decomposability of Pompeiu-like functions}

The techniques for proving the main results of this section are the modifications of the differentiable functions $f: J \rightarrow \mathbb{R}$ on some dense open subsets of $J$ such that the resulting function is still differentiable but closer to being Pompeiu-like. We refer to such a procedure as plastic surgery of f. Proposition 1.3.6, which gives us a replacement piece, and Proposition 1.3.7, which is our actual plastic surgery kit, are both applied in Lemma 1.3.8. In particular, Lemma 1.3.8 facilitates plastic surgeries locally on every nontrivial compact interval and implants Pompeiu-likeness on any (dense) open subset of $J$.

In Section 1.3.2, we only need (iii) of Lemma 1.3.8, while (ii), (iv) and (v) are used in Section 1.3.3 to create various examples of differentiable monsters, and (i) is especially used in Theorem 1.4.3.

Lemma 1.3.8. Let $f: J \rightarrow \mathbb{R}$ be any differentiable function. For every $\varepsilon>0$, closed $P \subseteq J$, and $P$-filling $\xi:[0,1] \rightarrow \mathbb{R}$, there exists a differentiable $g: J \rightarrow \mathbb{R}$ that is Pompeiu-like on every connected component of $J \backslash P$ such that

$$
\|g-f\|_{\infty} \leq \varepsilon, \quad g \uparrow_{P}=f \uparrow_{P}, \quad g^{\prime} \uparrow_{P}=f^{\prime} \uparrow_{P}, \quad\left\|g^{\prime}\right\|_{\infty} \leq\left\|f^{\prime}\right\|_{\infty}\left\|\xi^{\prime}\right\|_{\infty},
$$

and

(i) if $f$ is nowhere-constant on $J \backslash P$ and $\xi$ is nowhere-monotone, then $g$ is nowhere-monotone on $J \backslash P$.

In addition, when $P \subseteq J$ is required to be nowhere-dense, we have not only $g \in \mathcal{P}(J)$ but also the following easy consequences.

(ii) If $f$ is nowhere-constant and $\xi$ is nowhere-monotone, then $g$ is nowhere-monotone on $J$.

(iii) If $f$ and $\xi$ are (strictly) increasing, then so is $g$.

(iv) If $f$ has a bounded total variation, then so has $g$.

(v) If $\alpha$ and $\beta$ are differentiable and strictly increasing on $J$, then there exist strictly increasing $\bar{\alpha}, \bar{\beta} \in \mathcal{P}(J)$ satisfying (1.6) such that $\bar{\alpha}-\bar{\beta}$ is nowhere-monotone. 
Proof. Increasing $P$ slightly, if necessary, we can assume that each connected component of $J \backslash P$ is an interval $(a, b)$ with $a, b \in P$ to start with. Indeed, if $\left\langle c_{k}\right\rangle_{k \in \mathbb{Z}}$ is a strictly increasing sequence in $J$ such that

$$
\lim _{k \rightarrow-\infty} c_{k}=\inf J, \text { and } \lim _{k \rightarrow \infty} c_{k}=\sup J
$$

and $C$ is the closure of $\left\{c_{k}: k \in \mathbb{Z}\right\}$ in $J$, then $\bar{P}:=P \cup C$ has such a property. Moreover, if $g$ is in the lemma for $\bar{P}$, then it is easy to see that $g$ also satisfies our lemma for $P$.

Let $\mathcal{I}$ be the collection of all nonempty connected components of $J \backslash P$. Also, let $H_{P}$ be the smooth function satisfying (i) in Proposition 1.3.7 with $\left\|H_{P}\right\|_{\infty} \leq \varepsilon$. For every $I \in \mathcal{I}$, let $\left\langle z_{k}^{I}\right\rangle_{k \in \mathbb{Z}}$ be a strictly increasing sequence in $I$ such that

$$
\lim _{k \rightarrow-\infty} z_{k}^{I}=\inf I, \text { and } \lim _{k \rightarrow \infty} z_{k}^{I}=\sup I .
$$

Applying Lebesgue's number lemma to $f \uparrow_{\left[z_{k}^{I}, z_{k+1}^{I}\right]}$ and $\hat{\varepsilon}=\frac{1}{2\|\xi\|_{\infty}} \min _{t \in\left[z_{k}^{I}, z_{k+1}^{I}\right]} H_{P}(t)$, we can refine the sequence $\left\langle z_{k}^{I}\right\rangle_{k \in \mathbb{Z}}$, if necessary, so that for every $k \in \mathbb{Z}$, we also have

$$
|f(x)-f(y)|<\hat{\varepsilon} \leq \frac{1}{2\|\xi\|_{\infty}} H_{P}(t) \quad \text { for all } x, y, t \in\left[z_{k}^{I}, z_{k+1}^{I}\right]
$$

In addition, if $f$ is nowhere-constant, then we can further refine $\left\langle z_{k}^{I}\right\rangle_{k \in \mathbb{Z}}$ so that $f\left(z_{k}^{I}\right) \neq f\left(z_{k+1}^{I}\right)$ for every $k \in \mathbb{Z}$.

For each $I \in \mathcal{I}$ and $k \in \mathbb{Z}$, choose an increasing linear surjection $\mathrm{L}_{k}^{I}:\left[z_{k}^{I}, z_{k+1}^{I}\right] \rightarrow[0,1]$. Then

$$
g:=f \uparrow_{P} \cup \bigcup_{I \in \mathcal{I}} \bigcup_{k \in \mathbb{Z}}\left(f\left(z_{k+1}^{I}\right)-f\left(z_{k}^{I}\right)\right)\left(\xi \circ \mathrm{L}_{k}^{I}\right)+f\left(z_{k}^{I}\right)
$$

is as needed.

Indeed, $g$ is differentiable on any $I \in \mathcal{I}$, since for every $k \in \mathbb{Z}$, the values of $g$ and its derivatives at $z_{k+1}^{I}$ are equal to $f\left(z_{k+1}^{I}\right)$ and 0 respectively. Thus, by (ii) of Proposition 1.3.7, to finish the proof of the main part of the lemma, it is enough to show $|g-f| \leq H_{P}$ on every $I \in \mathcal{I}$. This is the case, 
since using (1.7) for every $x \in\left[z_{k}^{I}, z_{k+1}^{I}\right]$, we have

$$
\begin{aligned}
|g(x)-f(x)| & \leq\left|g(x)-f\left(z_{k}^{I}\right)\right|+\left|f\left(z_{k}^{I}\right)-f(x)\right| \\
& =\left|\left(f\left(z_{k+1}^{I}\right)-f\left(z_{k}^{I}\right)\right)\left(\xi \circ \mathrm{L}_{k}^{I}\right)(x)\right|+\left|f\left(z_{k}^{I}\right)-f(x)\right| \\
& \leq\left|f\left(z_{k+1}^{I}\right)-f\left(z_{k}^{I}\right)\right|\|\xi\|_{\infty}+\left|f\left(z_{k}^{I}\right)-f(x)\right|\|\xi\|_{\infty} \\
& <2 \hat{\varepsilon}\|\xi\|_{\infty} \leq H_{P}(x) .
\end{aligned}
$$

To see $\left\|g^{\prime}\right\|_{\infty} \leq\left\|f^{\prime}\right\|_{\infty}\left\|\xi^{\prime}\right\|_{\infty}$, notice that for every $I \in \mathcal{I}, k \in \mathbb{Z}$, and $x \in\left[z_{k}^{I}, z_{k+1}^{I}\right]$, we have

$$
\begin{aligned}
\left|g^{\prime}(x)\right| & =\left|\left(f\left(z_{k+1}^{I}\right)-f\left(z_{k}^{I}\right)\right)\left(\xi \circ \mathrm{L}_{k}^{I}\right)^{\prime}(x)\right| \\
& =\left|f\left(z_{k+1}^{I}\right)-f\left(z_{k}^{I}\right)\right| \cdot\left|\xi^{\prime}\left(\mathrm{L}_{k}^{I}(x)\right)\right| \cdot \frac{1}{z_{k+1}^{I}-z_{k}^{I}} \\
& \leq\left|\frac{f\left(z_{k+1}^{I}\right)-f\left(z_{k}^{I}\right)}{z_{k+1}^{I}-z_{k}^{I}}\right|\left\|\xi^{\prime}\right\|_{\infty} \leq\left\|f^{\prime}\right\|_{\infty}\left\|\xi^{\prime}\right\|_{\infty} .
\end{aligned}
$$

So, since $\left\|\xi^{\prime}\right\|_{\infty} \geq 1$ implies that $\left\|f^{\prime} \uparrow_{P}\right\|_{\infty} \leq\left\|f^{\prime}\right\|_{\infty} \leq\left\|f^{\prime}\right\|_{\infty}\left\|\xi^{\prime}\right\|_{\infty}$, we have the last inequality:

$$
\left\|g^{\prime}\right\|_{\infty}=\max \left\{\left\|f^{\prime} \uparrow_{P}\right\|_{\infty}, \sup _{I \in \mathcal{I}, k \in \mathbb{Z}}\left\|g^{\prime} \uparrow_{\left[z_{k}^{I}, z_{k+1}^{I}\right]}\right\|_{\infty}\right\} \leq\left\|f^{\prime}\right\|_{\infty}\left\|\xi^{\prime}\right\|_{\infty} .
$$

This completes the proof of (1.6).

Part (iii) is clear when we use $\xi:=\varphi$ from Proposition 1.3.6 in the above construction. Similarly, (i) and (ii) hold if we use $\xi:=\sigma$ from Proposition 1.3.6 in the construction. Indeed, our requirement $f\left(z_{k}^{I}\right) \neq f\left(z_{k+1}^{I}\right)$ ensures that both sets $\left[g^{\prime}>0\right]$ and $\left[g^{\prime}<0\right]$ are dense in any interval $\left[z_{k}^{I}, z_{k+1}^{I}\right]$, so these sets are dense not only in every connected component of $J \backslash P$ but also in the entire $J$ when $P$ is additionally assumed to be nowhere-dense.

To see (iv), let $\operatorname{TV}_{g}(J)$ denote the total variation of $g$ on $J$. First notice that $g$ is obtained from modifying $f$ on $J \backslash P=\bigcup_{I \in \mathcal{I}} \bigcup_{k \in \mathbb{Z}}\left[z_{k}^{I}, z_{k+1}^{I}\right]$, and thus

$$
\begin{aligned}
\operatorname{TV}_{g}(J) & \leq \operatorname{TV}_{f}(J)+\sum_{I \in \mathcal{I}} \sum_{k \in \mathbb{Z}} \operatorname{TV}_{g}\left(\left[z_{k}^{I}, z_{k+1}^{I}\right]\right) \\
& =\operatorname{TV}_{f}(J)+\sum_{I \in \mathcal{I}} \sum_{k \in \mathbb{Z}}\left|f\left(z_{k+1}^{I}\right)-f\left(z_{k}^{I}\right)\right| \operatorname{TV}_{\xi}[0,1] \\
& \leq \operatorname{TV}_{f}(J)+\operatorname{TV}_{f}(J) \operatorname{TV}_{\xi}[0,1]<\infty,
\end{aligned}
$$

is as needed. 
For $(\mathrm{v})$, we use P-fillings $\xi_{\alpha}:=\varphi$ and $\xi_{\beta}:=\psi$ from Proposition 1.3.6 to simultaneously modify functions $\alpha$ and $\beta$ on $J \backslash P$ to obtain their counterparts $\bar{\alpha}, \bar{\beta} \in \mathcal{P}(J)$ which, by (i), will be (strictly) increasing. Specifically, for every $I \in \mathcal{I}$, we refine the sequence $\left\langle z_{k}^{I}\right\rangle_{k \in \mathbb{Z}}$ so that (1.7) is satisfied with both $\alpha$ and $\beta{ }^{13}$ Using the same partition $\left\langle z_{k}^{I}\right\rangle_{k \in \mathbb{Z}}$ of $I \in \mathcal{I}$ to modify " $\alpha$ with $\varphi$ " and " $\beta$ with $\psi$ " means that $\bar{\alpha}-\bar{\beta}$ is the result of the modification of $\alpha-\beta$ with a nowhere-monotone P-filling

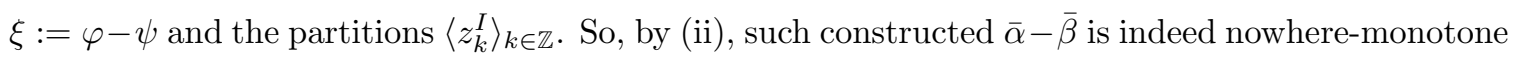
on $J$.

In Lemma 1.3.9, a sequence of straight lines $\left\{\mathcal{L}_{n}\right\}_{n \in \mathbb{N}}$ will be turned into a sequence of increasing Pompeiu-like functions $\left\{\ell_{n}\right\}_{n \in \mathbb{N}}$. The purpose is to stack up $\left\{\ell_{n}\right\}_{n \in \mathbb{N}}$ to obtain an increasing Pompeiu-like function with its derivative being sufficiently large on a designated dense subset. Details of this construction are shown in the lemma below.

Lemma 1.3.9. Let $f: J \rightarrow \mathbb{R}$ be any Pompeiu-like function. For every $M>0$, there exists a strictly increasing $g_{M} \in \mathcal{P}(J)$ such that

$$
g_{M}^{\prime} \geq f^{\prime} \text { on }\left[f^{\prime}<M\right]
$$

Proof. For every $n \in \mathbb{N}$, let $\mathcal{L}_{n}$ be the restriction to $J$ of the straight line through the origin and of slope $\frac{M}{2^{n}}$, and let $P_{n}$ be the closure of $\left[f^{\prime} \geq \frac{M}{2^{n}}\right]$ in $J$. By Proposition 1.3.3, each $P_{n}$ is nowhere-dense in $J$. With a fixed strictly increasing P-filling $\varphi:[0,1] \rightarrow \mathbb{R}$, which exists by Proposition 1.3.6, for every $n \in \mathbb{N}$, we use (iii) of Lemma 1.3 .8 to $f:=\mathcal{L}_{n}$ and $\varepsilon:=\frac{1}{2^{n}}$ to find an increasing Pompeiu-like $\ell_{n}: J \rightarrow \mathbb{R}$ such that

$$
\left\|\ell_{n}-\mathcal{L}_{n}\right\|_{\infty} \leq \frac{1}{2^{n}}, \ell_{n}^{\prime}\left\lceil P_{n}=\mathcal{L}_{n}^{\prime}\left\lceil P_{n}, \text { and }\left\|\ell_{n}^{\prime}\right\|_{\infty} \leq \frac{M}{2^{n}}\left\|\varphi^{\prime}\right\|_{\infty}\right.\right.
$$

We will show $g_{M}:=\sum_{n \in \mathbb{N}} \ell_{n}$ is as needed.

First, we consider the case when $J=[a, b]$ is compact. Then, for every $n \in \mathbb{N}$,

$$
\begin{aligned}
\left\|\ell_{n}\right\|_{\mathrm{C}^{1}} & =\left\|\ell_{n}\right\|_{\infty}+\left\|\ell_{n}^{\prime}\right\|_{\infty} \leq\left\|\ell_{n}-\mathcal{L}_{n}\right\|_{\infty}+\left\|\mathcal{L}_{n}\right\|_{\infty}+\left\|\ell_{n}^{\prime}\right\|_{\infty} \\
& \leq \frac{1}{2^{n}}+\frac{M \max \{|a|,|b|\}}{2^{n}}+\frac{M}{2^{n}}\left\|\varphi^{\prime}\right\|_{\infty}=\frac{C}{2^{n}}
\end{aligned}
$$

where $C:=1+M \max \{|a|,|b|\}+M\left\|\varphi^{\prime}\right\|_{\infty}$ is a constant. So, the partial sums sequence $\left\langle\sum_{n \leq k} \ell_{n}\right\rangle_{k \in \mathbb{N}}$ is Cauchy, as dominated by a geometric series. In particular, by Proposition 1.3.4, $g_{M}$ is well-defined and Pompeiu-like, that is, $g_{M} \in \mathcal{P}([a, b])$. Clearly, it is also increasing, as a limit of such functions. It remains to show $g_{M}^{\prime}(x) \geq f^{\prime}(x)$ whenever $x \in\left[f^{\prime}<M\right]$.

\footnotetext{
${ }^{13}$ For instance, we can require that $|\alpha(x)-\alpha(y)|+|\beta(x)-\beta(y)|<\frac{1}{2\left(\|\varphi\|_{\infty}+\|\psi\|_{\infty}\right)} H_{P}(t)$ for all $x, y, t \in\left[z_{k}^{I}, z_{k+1}^{I}\right]$.
} 
Indeed, if $x \in\left[f^{\prime} \leq 0\right]$, then we have $g_{M}^{\prime}(x) \geq 0 \geq f^{\prime}(x)$. On the other hand, if $x \in\left[0<f^{\prime}<M\right]$, then there exists a $k \in \mathbb{N}$ such that $x \in\left[\frac{M}{2^{k+1}} \leq f^{\prime}<\frac{M}{2^{k}}\right]$. Since $x \in \bigcup_{n=k+1}^{\infty} P_{n}$, we get the needed inequality:

$$
g_{M}^{\prime}(x) \geq \sum_{n=k+1}^{\infty} \ell_{n}^{\prime}(x)=\sum_{n=k+1}^{\infty} \mathcal{L}_{n}^{\prime}(x)=\sum_{n=k+1}^{\infty} \frac{M}{2^{n}}=\frac{M}{2^{k}}>f^{\prime}(x)
$$

This finishes the case when $J=[a, b]$.

The general case follows because $J$ can be written as the union of an increasing sequence $\left\langle\left[a_{n}, b_{n}\right]\right\rangle_{n \in \mathbb{N}}$ while $g_{M}^{\prime} \Upsilon_{\left[a_{n}, b_{n}\right]}$ is well defined and as needed for each $n$.

Theorem 1.3.1 is an easy consequence of Lemma 1.3.9.

Proof of Theorem 1.3.1. Let $f: J \rightarrow \mathbb{R}$ be any Pompeiu-like function such that $f^{\prime}$ is bounded either from above or from below. We need to represent $f$ as a difference of two increasing Pompeiu-like functions. Without loss of generality, we assume that $f^{\prime}$ is bounded above, as otherwise we may replace $f$ with $-f$. Let $M>0$ be a strict upper bound for $f^{\prime}$. By Lemma 1.3.9, there exists a strictly increasing $g_{M} \in \mathcal{P}(J)$ such that $g_{M}^{\prime} \geq f^{\prime}$ on $\left[f^{\prime}<M\right]=J$. Clearly, $g_{M}-f$ is an increasing differentiable function on $J$ as it has a nonnegative derivative and $g_{M}-f \in \mathcal{P}(J)$ by Proposition 1.3.4. Thus, the pair $g_{M}$ and $g_{M}-f$, that gives $f=g_{M}-\left(g_{M}-f\right)$, is as needed.

Note that there is no guarantee that $g_{M}-f$ is strictly increasing. If we want to write $f$ as a difference of two strictly increasing Pompeiu-like functions, we may simply add a strictly increasing Pompeiu-like function to both $g_{M}$ and $g_{M}-f$.

Proof of Corollary 1.3.2. Clearly, differentiability is necessary for Pompeiu-likeness. To prove the other implication, assume that $f=g-(g-f)$, where both $g$ and $g-f$ are increasing and differentiable, but not necessarily Pompeiu-like. Fix any $M>0$ and let $P_{M}$ be the closure of $\left[f^{\prime} \geq M\right]$ in $J$. We use (iii) of Lemma 1.3.8 to turn $g$ into an increasing Pompeiu-like $\tilde{g}: J \rightarrow \mathbb{R}$ such that $\tilde{g}^{\prime}\left\lceil P_{M}=g^{\prime}\left\lceil P_{M}\right.\right.$. Clearly, $f-\tilde{g} \in \mathcal{P}(J)$. We will show the derivative of $f-\tilde{g}$ is bounded above by $M$. Indeed,

- If $x \in\left[f^{\prime} \geq M\right]$, then $f^{\prime}(x)-\tilde{g}^{\prime}(x)=f^{\prime}(x)-g^{\prime}(x) \leq 0<M$.

- If $x \in\left[f^{\prime}<M\right]$, then $f^{\prime}(x)-\tilde{g}^{\prime}(x) \leq f^{\prime}(x)<M$.

As $f-\tilde{g}$ satisfies the premise of Theorem 1.3.1, we can write $f-\tilde{g}=\alpha-\beta$, where $\alpha$ and $\beta$ are both increasing Pompeiu-like. Since $\alpha+\tilde{g}$ is also an increasing Pompeiu-like function, the pair $\alpha+\tilde{g}$ and $\beta$, that gives $f=(\alpha+\tilde{g})-\beta$, is as needed.

The last result of this section shows that for every Pompeiu-like function $f: J \rightarrow \mathbb{R}$, and thus for every differentiable monster, the family $\mathcal{I}$ of all nonempty open intervals $I \subseteq J$ for which $f\lceil I$ 
is a difference of two increasing Pompeiu-like functions has its union $\bigcup \mathcal{I}$ being dense in $J$. Thus, (Q1) has an "almost" positive answer.

Corollary 1.3.10. For every Pompeiu-like $f: J \rightarrow \mathbb{R}$ and nonempty open $U \subseteq J$, there exists a nonempty open interval $I \subseteq U$ such that $f \uparrow_{I}$ is a difference of two increasing Pompeiu-like functions on $I$.

Proof. Fix any $M>0$. By Proposition 1.3.3, the set $\left[f^{\prime} \geq M\right]$ is nowhere-dense in $J$. So, there exists a nonempty open interval $I \subseteq U \backslash\left[f^{\prime} \geq M\right]$. Since $f^{\prime}<M$ on $I$, the result follows from Theorem 1.3.1.

\subsubsection{Examples and discussion}

All functions considered in this section are assumed to be defined on a fixed nontrivial compact interval $[a, b] \subseteq \mathbb{R}$. Let $\mathrm{D}, \mathcal{P}$, and $\mathcal{M}$ denote, respectively, the collections of all differentiable functions, Pompeiu-like functions, and differentiable monsters. Clearly, $\emptyset \neq \mathcal{M} \subsetneq \mathcal{P} \subsetneq \mathrm{D}$, where the first inclusion is strict since there exists a strictly increasing Pompeiu-like function.

Consider also the following classes of functions from $[a, b]$ to $\mathbb{R}$ :

$$
\begin{aligned}
& \mathcal{V}:=\{f \in \mathrm{D}: \quad f \text { is of bounded variation }\} \\
& \mathcal{N}:=\left\{f \in \mathcal{V}: \text { all Dini derivatives of the total variation } \operatorname{TV}_{f}[a, x] \text { are finite }\right\}^{14} \\
& \mathcal{C}:=\{f \in \mathrm{D}: \quad f=\alpha-\beta \text { for some increasing } \alpha, \beta \in \mathrm{D}\} \\
& \mathcal{T}:=\left\{f \in \mathcal{V}: \operatorname{TV}_{f}[a, x] \text { is differentiable }\right\} \\
& \mathcal{B}:=\left\{f \in \mathrm{D}: f^{\prime} \text { is bounded either from above or below }\right\}
\end{aligned}
$$

Notice that, by Corollary 1.3.2, the class $\mathcal{C} \cap \mathcal{M}$ equals to the family of all differentiable monsters that can be represented as a difference of two increasing Pompeiu-like function, that is, precisely those differentiable monsters for which the answer to our (Q1) is positive. In the remainder of this section, we will relate this class to the other classes defined above.

We will start with the following remark, which will help us to relate classes $\mathcal{C}$ and $\mathcal{C} \cap \mathcal{M}$ to the other just defined classes.

Remark 1.3.11. $\mathcal{B} \cup \mathcal{T} \subsetneq \mathcal{C} \subseteq \mathcal{N} \subsetneq \mathcal{V}$

\footnotetext{
${ }^{14} \mathrm{D}^{+}, \mathrm{D}^{-}, \mathrm{D}_{+}, \mathrm{D}_{-}$denote the operators that calculates, respectively, the upper-right, upper-left, lower-right, lowerleft Dini derivatives of a function. $\operatorname{TV}_{f}[a, x]$ denotes the total variation function of $f$ starting at $a$. Similarly, $\mathrm{PV}_{f}[a, x]$ and $\mathrm{NV}_{f}[a, x]$ denote the positive variation function and the negative variation function respectively.
} 
Proof. The inclusion $\mathcal{N} \subseteq \mathcal{V}$ is obvious from the definitions of these classes. To see $\mathcal{B} \cup \mathcal{T} \subseteq \mathcal{C}$, fix an $f \in \mathcal{B} \cup \mathcal{T}$. We need to show $f \in \mathcal{C}$.

Indeed, if $f^{\prime}$ is bounded above by an $M>0$ and $\mathcal{L}_{M}$ is the restriction to $[a, b]$ of the straight line through the origin and of the slope $M$, then functions $\mathcal{L}_{M}$ and $\mathcal{L}_{M}-f$ are differentiable increasing and we have $f=\mathcal{L}_{M}-\left(\mathcal{L}_{M}-f\right) \in \mathcal{C}$. If $f^{\prime}$ is bounded below, then $(-f)^{\prime}$ is bounded above and, by the above argument $-f$, so also $f$, belongs to $\mathcal{C}$. Finally, if $f \in \mathcal{T}$, then maps $\operatorname{TV}_{f}[a, x]$ and $\mathrm{TV}_{f}[a, x]-f(x)$ are differentiable increasing and $f(x)=\mathrm{TV}_{f}[a, x]-\left(\mathrm{TV}_{f}[a, x]-f(x)\right) \in \mathcal{C}$. This completes the argument for $\mathcal{B} \cup \mathcal{T} \subseteq \mathcal{C}$.

To see $\mathcal{C} \subseteq \mathcal{N}$, we will show if $f \notin \mathcal{N}$, then $f \notin \mathcal{C}$. Notice that if $f$ is not even in $\mathcal{V}$, then $f$ is clearly not in $\mathcal{C}$. Thus, we start with an $f \in \mathcal{V} \backslash \mathcal{N}$ and any pair of increasing functions $\alpha$ and $\beta$ such that $f=\alpha-\beta$. In particular, if one of the four Dini derivative of $\operatorname{TV}_{f}[a, x]$ is infinite at some $c \in[a, b]$, we will show that $\alpha$ and $\beta$ cannot both be differentiable at $c$. Without loss of generality, we assume $\mathrm{D}^{+} \mathrm{TV}_{f}[a, x]=\mathrm{D}^{+} \mathrm{PV}_{f}[a, x]+\mathrm{D}^{+} \mathrm{NV}_{f}[a, x]$ is infinite at $c$. Note that the positive variation of $f+g$ is never less than the positive variation of $f$ whenever $g$ is increasing. Suppose $\mathrm{D}^{+} \mathrm{PV}_{f}[a, x]$ is infinite at $c$. For every $x>c$, we have

$$
\mathrm{PV}_{f}[a, x]-\mathrm{PV}_{f}[a, c]=\mathrm{PV}_{f}[c, x] \leq \mathrm{PV}_{f+\beta}[c, x]=\mathrm{PV}_{\alpha}[c, x]=\alpha(x)-\alpha(c),
$$

and thus,

$$
\infty=\limsup _{x \rightarrow c^{+}} \frac{\operatorname{PV}_{f}[a, x]-\mathrm{PV}_{f}[a, c]}{x-c} \leq \limsup _{x \rightarrow c^{+}} \frac{\alpha(x)-\alpha(c)}{x-c}
$$

This means $\alpha$ cannot be differentiable at $c$. Similarly, if $\mathrm{D}^{+} \mathrm{NV}_{f}[a, x]$ is infinite at $c$, then $\beta$ cannot be differentiable at $c$ either.

Finally, notice that Examples 1.3.12 and 1.3.13 justify, respectively, that the inclusions $\mathcal{B} \cup \mathcal{T} \subseteq \mathcal{C}$ and $\mathcal{N} \subseteq \mathcal{V}$ are strict.

The graph presented in Fig. 1.1 summarizes the inclusions $\mathcal{M} \subsetneq \mathcal{P} \subsetneq \mathrm{D}$ on the bottom row and those from Remark 1.3.11 in the right column. Note that $\sigma$ from Proposition 1.3.6 is a function in $\mathcal{B} \cap \mathcal{M}$. We do not know whether $\sigma$ from Proposition 1.3.6 has a differentiable total variation function or not. Answering Problem 1.3.17 may allow us to have a clearer look at $\operatorname{TV}_{\sigma}[0, x]$. We do not know whether $\mathcal{N} \backslash \mathcal{C}$ is empty or not, either. Perhaps, it may be an approach to Problem 1.3.15.

Example 1.3.12. There is a differentiable monster $\hat{g}:[-1,1] \rightarrow \mathbb{R}$ such that 


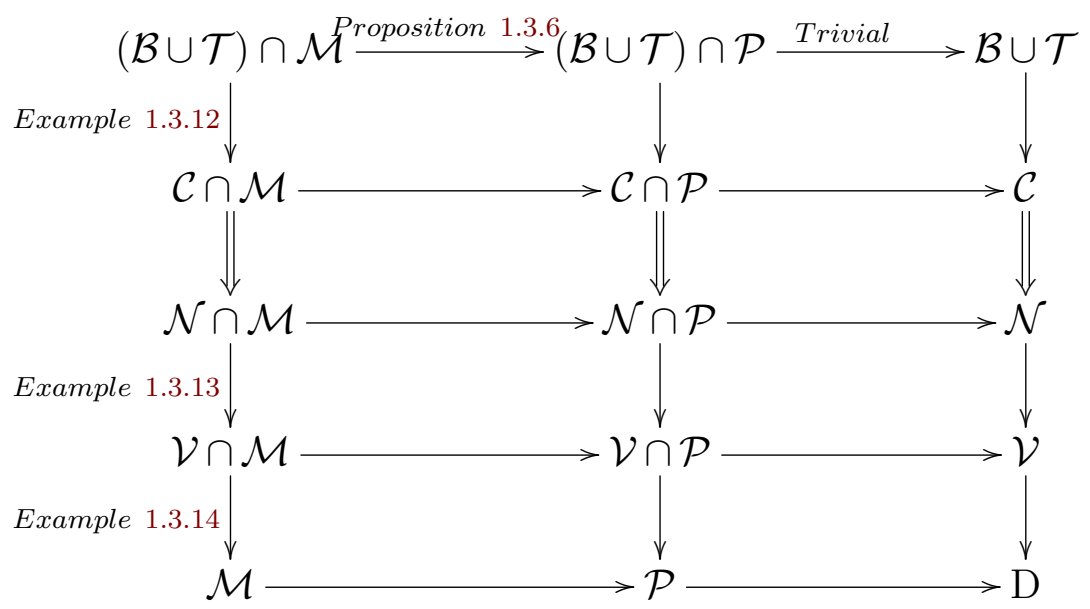

Figure 1.1: The inclusions, indicated by $\Rightarrow$ when strictness is unknown and by $\rightarrow$ when they are known to be strict, between the indicated classes. The indicated Examples justify strictness of all vertical inclusions between the associated rows. Similarly, the labels above the top horizontal arrows justify strictness of all horizontal inclusions between the associated columns.

(i) $\hat{g}$ is a difference of two increasing Pompeiu-like functions;

(ii) $\hat{g}^{\prime}$ is unbounded from both above and below near zero;

(iii) $\operatorname{TV}_{\hat{g}}[-1, x]$ is not differentiable at zero.

In particular, $\hat{g} \in \mathcal{C} \cap \mathcal{M} \backslash(\mathcal{B} \cup \mathcal{T})$, so that all indicated inclusions between the first two rows of Fig. 1.1 are strict.

Proof. Let $f:[0,1] \rightarrow \mathbb{R}$ be defined as

$$
f(x):= \begin{cases}x^{2} \cos (\pi / x) & \text { if } x \in(0,1] \\ 0 & \text { if } x=0 .\end{cases}
$$

It is well known and easy to see ${ }^{15}$ that

- $f$ is differentiable and $f^{\prime}(0)=0$;

- $\left\|f^{\prime}\right\|_{\infty}$ is bounded by $2+\pi$ as $f^{\prime}(x)=2 x \cos (\pi / x)+\pi \sin (\pi / x)$ for $x \neq 0$.

Notice that if $p_{n}=\frac{1}{n}$ for $n \in \mathbb{N}$, then $f\left(p_{n}\right)=\frac{(-1)^{n}}{n^{2}}$. Let $P:=\left\{p_{n}: n \in \mathbb{N}\right\} \cup\{0\}$. We use (ii) of Lemma 1.3.8 to the function $f$, the set $P$, and the nowhere-monotone $P$-filling $\sigma$ to obtain a differentiable monster $g$ such that $g \uparrow_{P}=f \uparrow_{P}, g^{\prime} \uparrow_{P}=f^{\prime} \uparrow_{P}$, and $\left\|g^{\prime}\right\|_{\infty} \leq\left\|f^{\prime}\right\|_{\infty}\left\|\sigma^{\prime}\right\|_{\infty}:=M$.

\footnotetext{
${ }^{15}$ Compare [47, A5].
} 
Let $\tau(x):=\mathrm{TV}_{g}[0, x]$. We have a lower bound ${ }^{16}$ and an upper bound ${ }^{17}$ for the difference quotient $\frac{\tau\left(p_{n}\right)-\tau(0)}{p_{n}-0}=\frac{\tau\left(p_{n}\right)}{p_{n}}$ :

$$
\frac{1}{n}+2 n \sum_{k=n+1}^{\infty} \frac{1}{k^{2}} \leq \frac{\tau\left(p_{n}\right)}{p_{n}} \leq M
$$

Moreover, by the integral test, ${ }^{18}$ we have

$$
2=\lim _{n \rightarrow \infty} 2 n \int_{n+1}^{\infty} \frac{1}{x^{2}} d x \leq \lim _{n \rightarrow \infty}\left(\frac{1}{n}+2 n \sum_{k=n+1}^{\infty} \frac{1}{k^{2}}\right) \leq \limsup _{n \rightarrow \infty} \frac{\tau\left(p_{n}\right)}{p_{n}} \leq M .
$$

It means that if $\tau^{\prime}(0)$ ever exists, then $2 \leq \tau^{\prime}(0) \leq M$. Define $\bar{g}:[-1,1] \rightarrow \mathbb{R}$ as

$$
\bar{g}(x):= \begin{cases}M g(x) & \text { if } x \in[0,1] \\ g(-x) & \text { if } x \in[-1,0) .\end{cases}
$$

Clearly, $\bar{g}$ is a differentiable monster and has a desired structure so that

$(\star)$ If $\hat{g}:[-1,1] \rightarrow \mathbb{R}$ agrees with $\bar{g}$ on $[-1,0] \cup P$ and $\hat{\tau}(x)=\operatorname{TV}_{\hat{g}}[-1, x]$, then $\hat{\tau}$ is not differentiable at 0 .

Indeed, otherwise both $\operatorname{limits}_{\lim _{x \rightarrow 0^{-}}} \frac{\hat{\tau}(x)}{x}$ and $\lim _{x \rightarrow 0^{+}} \frac{\hat{\tau}(x)}{x}$ exist and are equal. So $\lim _{x \rightarrow 0^{-}} \frac{\hat{\tau}(x)}{x}=$ $\tau^{\prime}(0) \leq M<2 M \leq M \tau^{\prime}(0)=\lim _{x \rightarrow 0^{+}} \frac{\hat{\tau}(x)}{x}$, a contradiction.

The property $\left(\star\right.$ ) ensures that $\bar{g}$ satisfies (iii). Also, since $\bar{g}^{\prime}$ is bounded, by Theorem 1.3.1, there exists a pair of strictly increasing Pompeiu-like functions $\alpha$ and $\beta$ such that $\alpha-\beta=\bar{g}$. In particular, $\bar{g}$ satisfies (i) and (iii). However, there is no reason for it to satisfy (ii). Thus, to ensure (ii), we need further modify functions $\alpha$ and $\beta$.

For every $n \in \mathbb{N}$, let $\left[a_{n}, b_{n}\right]$ be the middle third subinterval of $\left[p_{n+1}, p_{n}\right]$, and let $A:=\left\{a_{n}: n \in \mathbb{N}\right\}$ and $B:=\left\{b_{n}: n \in \mathbb{N}\right\}$. Notice that there exist increasing differentiable $\gamma, \delta:[-1,1] \rightarrow \mathbb{R}$ such that

- $\gamma(x)=0=\delta(x)$ for every $x \in[-1,0]$ and $\gamma\left(p_{n}\right)=\delta\left(p_{n}\right)$ for every $n \in \mathbb{N}$;

- $\gamma^{\prime}=0$ on $P \cup B$ and $\lim _{n \rightarrow \infty} \gamma^{\prime}\left(a_{n}\right)=\infty$;

- $\delta^{\prime}=0$ on $P \cup A$ and $\lim _{n \rightarrow \infty} \delta^{\prime}\left(b_{n}\right)=\infty$.

For example, if $\varepsilon_{n}=\frac{1}{3^{n} n(n+1)}$ and $h_{n}(x)=9^{n}(2 n+1) \operatorname{dist}\left(x,\left[a_{n}-\varepsilon_{n}, a_{n}+\varepsilon_{n}\right]^{c}\right)$ (see Fig. 1.2), then the function $\gamma(x):=\int_{-1}^{x} \sum_{n=1}^{\infty} h_{n}(t) d t$ is as needed. ${ }^{19}$ Function $\delta$ can be defined similarly.

${ }^{16} \tau\left(p_{n}\right)=\mathrm{TV}_{g}\left[0, p_{n}\right] \geq \sum_{k=n}^{\infty}\left|g\left(p_{k}\right)-g\left(p_{k+1}\right)\right|=\left|f\left(p_{n}\right)\right|+2 \sum_{k=n+1}^{\infty}\left|f\left(p_{k}\right)\right|$.

${ }^{17} \tau\left(p_{n}\right)=\operatorname{TV}_{g}\left[0, p_{n}\right] \leq\left\|g^{\prime}\right\|_{\infty}\left(p_{n}-0\right) \leq\left\|f^{\prime}\right\|_{\infty}\left\|\sigma^{\prime}\right\|_{\infty} p_{n}=M p_{n}$, where the second inequality is justified by 


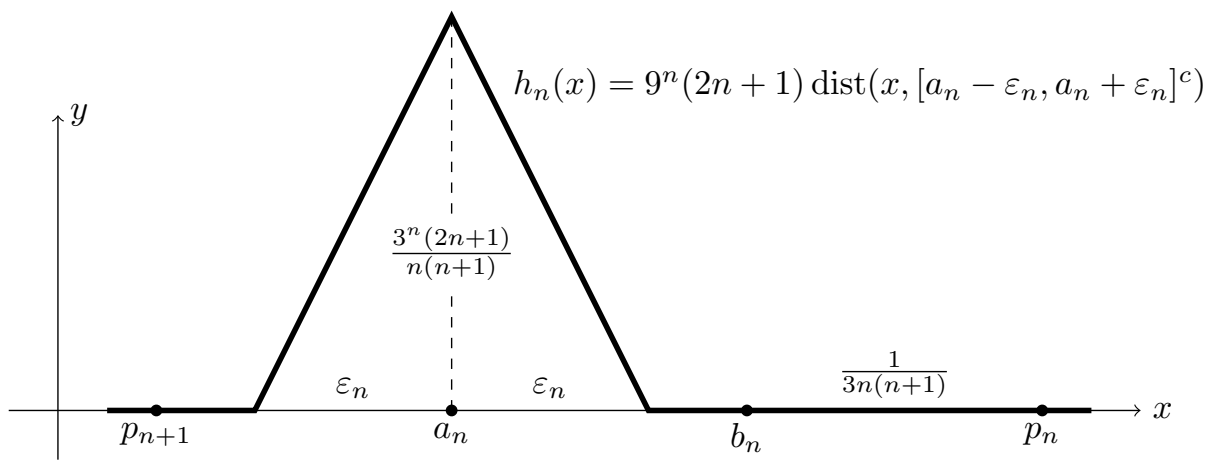

Figure 1.2: An illustration of $h_{n}$ on $\left[p_{n+1}, p_{n}\right]$ used in the construction of $\gamma$ in Example 1.3.12.

Apply (v) of Lemma 1.3.8 to the nowhere-dense closed set $P \cup A \cup B \subseteq[0,1]$ and functions $(\alpha+\gamma) \uparrow_{[0,1]}$ and $(\beta+\delta) \uparrow_{[0,1]}$ to find a pair of strictly increasing Pompeiu-like functions $\bar{\alpha}, \bar{\beta}:[0,1] \rightarrow$ $\mathbb{R}$ such that $\bar{\alpha}-\bar{\beta}$ is a differentiable monster. Define

$$
\hat{\alpha}(x):=\left\{\begin{array}{ll}
\bar{\alpha}(x) & \text { if } x \in[0,1] \\
\alpha(x) & \text { if } x \in[-1,0)
\end{array} \quad \text { and } \quad \hat{\beta}(x):= \begin{cases}\bar{\beta}(x) & \text { if } x \in[0,1] \\
\beta(x) & \text { if } x \in[-1,0) .\end{cases}\right.
$$

It is easy to see that such extensions are still strictly increasing and Pompeiu-like. We claim that $\hat{g}:=\hat{\alpha}-\hat{\beta}$ is as needed.

Indeed, $\hat{g}$ is nowhere-monotone, since the choice of $\bar{\alpha}$ and $\bar{\beta}$ ensures this on $[0,1]$, while $\hat{g}=\bar{g}$ on $[-1,0]$ and $\bar{g}$ is nowhere-monotone. So, $\hat{g}$ is a differentiable monster satisfying (i). It satisfies (iii) by $(\star)$ as $\hat{g}=\bar{g}$ on $[-1,0] \cup P$. Finally, to see that $\hat{g}$ satisfies (ii), notice that $\delta^{\prime}\left(a_{n}\right)=0$, so

$$
\hat{g}^{\prime}\left(a_{n}\right)=(\alpha+\gamma)^{\prime}\left(a_{n}\right)-(\beta+\delta)^{\prime}\left(a_{n}\right) \geq-\left\|\bar{g}^{\prime}\right\|_{\infty}+\gamma^{\prime}\left(a_{n}\right) \rightarrow_{n \rightarrow \infty} \infty
$$

and, as $\gamma^{\prime}\left(b_{n}\right)=0$,

$$
\hat{g}^{\prime}\left(b_{n}\right)=(\alpha+\gamma)^{\prime}\left(b_{n}\right)-(\beta+\delta)^{\prime}\left(b_{n}\right) \leq\left\|\bar{g}^{\prime}\right\|_{\infty}-\delta^{\prime}\left(b_{n}\right) \rightarrow_{n \rightarrow \infty}-\infty .
$$

Example 1.3.13. There is a differentiable monster $g:[0,1] \rightarrow \mathbb{R}$ such that

\footnotetext{
Lemma 1.3.8.

$18 \frac{1}{n+1}=\int_{n+1}^{\infty} \frac{1}{x^{2}} d x \leq \sum_{n+1}^{\infty} \frac{1}{k^{2}}$.

${ }^{19} \gamma^{\prime}\left(a_{n}\right)=9^{n}(2 n+1) \varepsilon_{n}=\frac{3^{n}(2 n+1)}{n(n+1)} \rightarrow_{n \rightarrow \infty} \infty$ is obvious. The bound $\varepsilon_{n} \leq \frac{p_{n}-p_{n+1}}{3}$ ensures $\gamma^{\prime}\left(p_{n}\right)=0=\gamma^{\prime}\left(b_{n}\right)$. Also, $\int_{p_{n+1}}^{p_{n}} \gamma(x) d x=9^{n}(2 n+1) \varepsilon_{n}^{2}=\frac{9^{n}(2 n+1)}{9^{n} n^{2}(n+1)^{2}}=\frac{2 n+1}{n^{2}(n+1)^{2}}=p_{n}^{2}-p_{n+1}^{2}$ ensures $\gamma\left(p_{n}\right)=p_{n}^{2}$. For differentiability at zero, $\limsup _{x \rightarrow 0} \frac{\gamma(x)-\gamma(0)}{x-0} \leq \lim _{n \rightarrow \infty} \frac{\gamma\left(p_{n}\right)}{p_{n+1}}=0$ justifies $\gamma^{\prime}(0)=0$.
} 
(i) $g$ is of bounded variation;

(ii) $\mathrm{D}^{+} \mathrm{TV}_{g}[0, x]=\infty$ at zero.

In particular, $g \in \mathcal{V} \cap \mathcal{M} \backslash \mathcal{N}$, so that all indicated inclusions between the third and the forth rows of Fig. 1.1 are strict.

Proof. Let $f:[0,1] \rightarrow \mathbb{R}$ be a function defined as

$$
f(x):= \begin{cases}x^{3 / 2} \cos (\pi / x) & \text { if } x \in(0,1] \\ 0 & \text { if } x=0 .\end{cases}
$$

It is well known and easy to see ${ }^{20}$ that

- $f$ is differentiable with $f^{\prime}(0)=0$;

- $f$ is of bounded variation and, in fact, absolutely continuous.

Notice that if $p_{n}=\frac{1}{n}$ for $n \in \mathbb{N}$, then $f\left(p_{n}\right)=\frac{(-1)^{n}}{n^{3 / 2}}$. Let $P:=\left\{p_{n}: n \in \mathbb{N}\right\} \cup\{0\}$. The function $g$ is obtained by using (ii) of Lemma 1.3 .8 to the above defined $f$, set $P$, and a nowhere-monotone P-filling which exists by Proposition 1.3.6. Then parts (ii) and (iv) of Lemma 1.3.8 imply that $g \in \mathcal{M} \cap \mathcal{V}$. Moreover, if $\tau(x):=\mathrm{TV}_{g}[-1, x]$, then, by $g \uparrow_{P}=f \uparrow_{P}$,

$$
\tau\left(p_{n}\right) \geq \sum_{k=n}^{\infty}\left|f\left(p_{k}\right)-f\left(p_{k+1}\right)\right|=\left|f\left(p_{n}\right)\right|+2 \sum_{k=n+1}^{\infty}\left|f\left(p_{k}\right)\right|=\frac{1}{n^{3 / 2}}+2 \sum_{k=n+1}^{\infty} \frac{1}{k^{3 / 2}}
$$

and, by the integral test,

$$
\begin{aligned}
D^{+} \tau(0) \geq \limsup _{n \rightarrow \infty} \frac{\tau\left(p_{n}\right)-\tau(0)}{p_{n}-0} & \geq \limsup _{n \rightarrow \infty} \frac{\frac{1}{n^{3 / 2}}+2 \sum_{k=n+1}^{\infty} \frac{1}{k^{3 / 2}}}{\frac{1}{n}} \\
& \geq 0+2 \lim _{n \rightarrow \infty} n \int_{n+1}^{\infty} x^{-3 / 2} d x=\infty
\end{aligned}
$$

So, $g$ is as needed.

Example 1.3.14. There is a differentiable monster $g:[0,1] \rightarrow \mathbb{R}$ which is not of bounded variation. In particular, $g \in \mathcal{M} \backslash \mathcal{V}$, so that all indicated inclusions between the last two rows of Fig. 1.1 are strict.

\footnotetext{
${ }^{20}$ Compare [47, A14].
} 
Proof. Let $f:=[0,1] \rightarrow \mathbb{R}$ be a function defined as

$$
f(x):= \begin{cases}x^{2} \cos \left(\pi / x^{2}\right) & \text { if } x \in(0,1] \\ 0 & \text { if } x=0 .\end{cases}
$$

It is well known and easy to see that $f$ is differentiable but not of bounded variation. ${ }^{21}$ Notice that if $q_{n}=\frac{1}{n^{1 / 2}}$ for $n \in \mathbb{N}$, then $f\left(q_{n}\right)=\frac{(-1)^{n}}{n}$. Let $P:=\left\{p_{n}: n \in \mathbb{N}\right\} \cup\{0\}$ and $g$ be obtained by using (ii) of Lemma 1.3.8 to the above defined $f$, set $P$, and a nowhere-monotone P-filling. Then $g \in \mathcal{M}$. To see that $g$ (and also $f$ ) is not bounded variation it is enough to notice that

$$
\mathrm{TV}_{g}[0,1] \geq \sum_{n=1}^{\infty}\left|g\left(p_{k}\right)-g\left(p_{k+1}\right)\right|=1+2 \sum_{n=1}^{\infty} p_{n}^{2}=1+2 \sum_{n=1}^{\infty} \frac{1}{n}=\infty
$$

\subsubsection{Related open problems}

When is a differentiable monster or even a Pompeiu-like function on a nontrivial compact $[a, b] \subseteq \mathbb{R}$ a difference of two increasing Pompeiu-like functions? As a characterization is found in Corollary 1.3.2, we believe it is worthwhile to state the following classical-looking problems.

Problem 1.3.15. What is a characterization of real-valued differentiable functions of bounded variation on $[a, b] \subseteq \mathbb{R}$ that is a difference of two increasing differentiable functions.

This is, basically, (Q2) when Pompeiu-likeness is replaced with differentiability, and we also refer to "being a difference of two increasing differentiable functions" as a differentiable Jordanlike decomposition. See Remark 1.3.11 for one necessary condition $\mathcal{N}$ and two strictly sufficient conditions $\mathcal{B}$ and $\mathcal{T}$. Is $\mathcal{N}$ also sufficient? Probably not. How far is it away from being sufficient? We do not know yet. Perhaps, there can be a stronger sufficient condition. Regarding $\mathcal{T}$, our next problem is about those total variation functions.

Problem 1.3.16. What is a characterization of real-valued differentiable functions of bounded variation on $[a, b] \subseteq \mathbb{R}$ that has a differentiable total variation function?

One might suspect that $f$ and $\operatorname{TV}_{f}[a, x]$ would have some connection in differentiability. Unfortunately, the known results said nay. Let $f:[a, b] \rightarrow \mathbb{R}$ be a function of bounded variation. On the good side, $f$ and $\mathrm{TV}_{f}[a, x]$ do share the same continuity. ${ }^{22}$ However, differentiability has a different story.

\footnotetext{
${ }^{21}$ Compare [34, 6, chapter 3] or [70, problem 10, chapter 5].

${ }^{22}$ See [3, theorem 6.14$]$ for a proof.
} 
In fact, Examples 1.3.12 and 1.3.13 have provided differentiable functions with non-differentiable total variation functions. More generally, see [38, theorem 1] for a differentiable $f$ on $[0,1]$ that has a non-differentiable total variation function, and see [38, theorem 2] for a non-differentiable $f$ on $[0,1]$ that has a differentiable total variation function.

To introduce our next problem, we must first recall what we had in Proposition 1.3.6. $\varphi$ and $\psi$ are two strictly increasing Pompeiu-like function from $[0,1]$ onto $[0,1]$ such that $f:=\varphi-\psi$ is nowhere-monotone. We also wonder what its total variation function $\operatorname{TV}_{f}[0, x]$ looks like. Is it differentiable? Probably not. We know that $\operatorname{TV}_{f}[0, x] \leq(\varphi+\psi)(x)$ for every $x \in[0,1]$. How far are they away from each other? We suspect Problem 1.3.17 might be related to the unknown sufficiency of $\mathcal{N}$ mentioned earlier.

Problem 1.3.17. Let $f:[a, b] \rightarrow \mathbb{R}$ be a differentiable function. If there exists a pair of strictly increasing differentiable functions $\alpha$ and $\beta$ such that $f=\alpha-\beta$, then is there a method to obtain the total variation function of $f$ by reducing $\alpha+\beta$ ?

Notice that the derivative of a nowhere-constant Pompeiu-like function must be discontinuous on a dense set. Can it be approximately continuous? Definitely. Using [11, theorem 6.5 , chapter 2], we can construct a strictly increasing Pompeiu-like function with a bounded approximately continuous derivative. $^{23}$ On the other hand, we know that $G:\left(t^{\prime}, t^{\prime \prime}\right) \rightarrow\left(x^{\prime}, x^{\prime \prime}\right)$ defined as the inverse of (1.4) has a bounded derivative. Is it also approximately continuous? Our last problem is as follows.

Problem 1.3.18. Does the strictly increasing Pompeiu-like function constructed by Pompeiu himself in [67] have a approximately continuous derivative?

\subsection{Monstrous Extentions}

So far we have had two paradoxical examples of real functions, the Weierstrass's monsters and the differentiable monsters. In addition, we consider another paradoxical example $\mathfrak{f}$, first constructed in a 2016 paper [18] of K. Ciesielski and J. Jasinski. ${ }^{24}$ This $\mathfrak{f}: \mathfrak{X} \rightarrow \mathfrak{X}$ is an auto-diffeomorphism on a compact perfect subset $\mathfrak{X}$ of $\mathbb{R}$ with $\mathfrak{f}^{\prime}(x)=0$ for all $x \in \mathfrak{X} .^{25}$ Thus, $\mathfrak{f}$ is shrinking at every $x \in \mathfrak{X}$ and so, one would expect that the diameter of $\mathfrak{f}[\mathfrak{X}]$ should be smaller than that of $\mathfrak{X}$, which evidently is not the case. Of course, $\mathfrak{X}$ must have Lebesgue measure zero, since $\mathfrak{f}^{\prime} \equiv 0$ implies that $\mathfrak{f}[\mathfrak{X}]$ must

\footnotetext{
${ }^{23}$ Let $E$ be an $F_{\sigma}$-inner approximation of $\mathbb{R} \backslash \mathbb{Q}$ with full measure. There exists an approximately continuous function $f$ such that $0<f(x) \leq 1$ for all $x \in E$ and $f(x)=0$ for all $x \notin \mathbb{R} \backslash E$. Note that $f$ is a derivative, and thus its primitive is a strictly increasing Pompeiu-like function.

${ }^{24}$ Since then, the construction was further generalized, in [9], and simplified in [17].

${ }^{25} \mathrm{~A}$ perfect set is a closed set that has no isolated points. In other words, $P \subseteq \mathbb{R}$ is perfect if and only if $P=P$, where $P^{\prime}$ is the derived set of $P$, that is, the collection of all accumulation points of $P$.
} 
have measure zero. ${ }^{26}$ The construction of such an $\mathfrak{f}$ in [17] is simple enough to be described in few lines. Specifically, it can be defined as

$$
\mathfrak{f}:=h \circ \sigma \circ h^{-1}
$$

from $\mathfrak{X}:=h\left[2^{\omega}\right]$ onto itself, where $\sigma: 2^{\omega} \rightarrow 2^{\omega}$ is the add-one-and-carry adding machine ${ }^{27}$ and the map $h: 2^{\omega} \rightarrow 2^{\omega}$ is defined via formula $h(s):=\sum_{n=0}^{\infty} 2 s_{n} 3^{-(n+1) N\left(s \uparrow_{n}\right)}$, where $N\left(s \uparrow_{n}\right)$ is given by $N\left(s \uparrow_{n}\right):=\sum_{i<n-1} s_{i} 2^{i}+\left(1-s_{n-1}\right) 2^{n-1}+2^{n}$. Notice that $\mathfrak{X}=h\left[2^{\omega}\right]$ is a subset of the Cantor ternary set $\mathfrak{C}$ in $[0,1]$.

The goal of Section 1.4 is to pack the three paradoxical examples into two functions. In particular, Corollary 1.4.4 extends $\mathfrak{f}$ to a differentiable monster, and Theorem 1.4.5 extends $\mathfrak{f}$ to a Weierstrass's monster. Both proofs rely on a differentiable function $\overline{\mathfrak{f}}: \mathbb{R} \rightarrow \mathbb{R}$ extending $\mathfrak{f}$, which existence is provided by Jarník's extension theorem.

Proposition 1.4.1 (Jarník). Every differentiable function $f: P \rightarrow \mathbb{R},{ }^{28}$ where $P \subseteq \mathbb{R}$ is closed, admits a differentiable extension $\bar{f}: \mathbb{R} \rightarrow \mathbb{R}$.

In 1923, V. Jarník proved that every differentiable function $f$ from a closed subset $P$ of $\mathbb{R}$ into $\mathbb{R}$ has a differentiable extension $\bar{f}: \mathbb{R} \rightarrow \mathbb{R}$. An interesting story of this result being forgotten and rediscovered is described in details in [15]. ${ }^{29}$ In short, Jarník's full paper with this result [42], written in Czech, and its announcement [43] in French, with a sketch of construction, were published in rather obscure journals. So, the theorem was unnoticed by the mathematical community until the mid 1980's, when it was cited in [5]. In the meantime, the theorem was rediscovered in 1974 by G. Petruska and M. Laczkovich [66] and further elaborated on in 1984 by J. Mařík [57].

In a 2012 paper [51], M. Koc and L. Zajíček proved a version of Jarník's extension theorem showing that an extension $f$ of $\varphi$ can be, on the set $\mathbb{R} \backslash P$, as good as possible, that is, $C^{\infty}$. In the opposite direction, K. Ciesielski and C.-H. Pan proved in [21] that this $f$ can also be, on $\mathbb{R} \backslash P$, as "bad" as possible, that is, nowhere-monotone.

\footnotetext{
${ }^{26}$ See e.g., [32, page 355].

${ }^{27}$ For $s=\left\langle s_{0}, s_{1}, s_{2}, \ldots\right\rangle \in 2^{\omega}$ it is defined: $\sigma(s):=\langle 0,0,0, \ldots\rangle$ when $s_{i}=1$ for all $i<\omega$ and, otherwise, $\sigma(s):=\left\langle 0,0, \ldots, 0,1, s_{k+1}, s_{k+2}, \ldots\right\rangle$, where $s_{k}=0$ and $s_{i}=1$ for all $i<k$.

${ }^{28}$ The differentiability of $f: P \rightarrow \mathbb{R}$ is understood as the existence of its derivative only on $P^{\prime}$, the accumulation points of $P$, that is, a function $f^{\prime}: P^{\prime} \rightarrow \mathbb{R}$ such that $f^{\prime}(p):=\lim _{x \rightarrow p, x \in P} \frac{f(x)-f(p)}{x-p}$ for every $p \in P^{\prime}$.

${ }^{29}$ Compare also [26, section 3.3].
} 


\subsubsection{Differentiable monsters extending $\mathfrak{f}$}

Before extending such an $\mathfrak{f}: \mathfrak{X} \rightarrow \mathfrak{X}$, notice that the perfect set $\mathfrak{X}$ is a subset of the Cantor ternary set $\mathfrak{C}$ and thus is nowhere-dense. In the statement of Theorem 1.4.3, we could have additionally assumed $P \subseteq \mathbb{R}$ to be nowhere-dense and use (ii) of Lemma 1.3.8 in its proof. Since the proof with or without nowhere-denseness is almost identical, we dropped it to align with the premise of Jarník's extension theorem, stated in Proposition 1.4.1, and the proof of Lemma 1.5.3 which shows the space of "bad" differentiable extensions is not empty.

In order to apply (i) of Lemma 1.3.8 to Jarník's differentiable extension, we first need to ensure its extended part is nowhere-constant, and the next lemma is taking care of it.

Lemma 1.4.2. If $O \subseteq \mathbb{R}$ is open, then there exists a differentiable $\tilde{h}: \mathbb{R} \rightarrow \mathbb{R}$ such that $\tilde{h} \uparrow_{\mathbb{R} \backslash O}=0$ and $\tilde{h}$ is nowhere-constant on $O$.

Proof. Lemma 1.4.2 is similar to and much simpler than Lemma 1.3.8. The major difference is that we start with a zero function $f$ on $\mathbb{R}$ and a fixed nowhere-constant differentiable $\xi:[0,1] \rightarrow \mathbb{R}$ with $\xi(0)=\xi^{\prime}(0)=0=\xi(1)=\xi^{\prime}(1)$ and $\|\xi\|_{\infty}<\infty .^{30}$

Let $\mathcal{I}$ be the collection of all nonempty connected components of $O$. Also, let $H_{\mathbb{R} \backslash O}$ be the smooth function satisfying (i) in Proposition 1.3.7. For every $I \in \mathcal{I}$, let $\left\langle z_{k}^{I}\right\rangle_{k \in \mathbb{Z}}$ be a strictly increasing sequence in $I$ such that

$$
\lim _{k \rightarrow-\infty} z_{k}^{I}=\inf I, \text { and } \lim _{k \rightarrow \infty} z_{k}^{I}=\sup I \text {. }
$$

For each $I \in \mathcal{I}$ and $k \in \mathbb{Z}$, choose an increasing linear surjection $\mathrm{L}_{k}^{I}:\left[z_{k}^{I}, z_{k+1}^{I}\right] \rightarrow[0,1]$ and let $M_{k}^{I}:=\left\|H_{\mathbb{R} \backslash O} \uparrow_{\left[z_{k}^{I}, z_{k+1}^{I}\right]}\right\|_{\infty}$. Then

$$
\tilde{h}:=f \uparrow_{\mathbb{R} \backslash O} \cup \bigcup_{I \in \mathcal{I}} \bigcup_{k \in \mathbb{Z}} \frac{M_{k}^{I}}{\|\xi\|_{\infty}}\left(\xi \circ \mathrm{L}_{k}^{I}\right)
$$

is as needed.

Theorem 1.4.3. If $P \subseteq \mathbb{R}$ is closed and $f: P \rightarrow \mathbb{R}$ is differentiable, then there exists a differentiable $g: \mathbb{R} \rightarrow \mathbb{R}$ extending $f$ such that $g$ is nowhere-monotone on every connected component of $\mathbb{R} \backslash P$.

Proof. Let $\bar{f}: \mathbb{R} \rightarrow \mathbb{R}$ be a differentiable extension of $f$, existing by Proposition 1.4.1. Let $O$ be the intersection of the interior of $\left[\bar{f}^{\prime}=0\right]$ and $\mathbb{R} \backslash P$. If $O$ is empty, then we can say that $\bar{f}$ is nowhere-constant on $\mathbb{R} \backslash P$. If not, we may easily make it so by adding a differentiable $\tilde{h}: \mathbb{R} \rightarrow \mathbb{R}$,

\footnotetext{
${ }^{30}$ For example, $\xi(x):=1-\cos (2 \pi x)$ on $[0,1]$ can be it with $\|\xi\|_{\infty}=2$.
} 
constructed in Lemma 1.4.2, to $\bar{f}$. Since $P$ is closed in $\mathbb{R}$, we can modify $\bar{f}$ on its extended part $\mathbb{R} \backslash P$ with (i) of Lemma 1.3 .8 to obtain a differentiable $g: \mathbb{R} \rightarrow \mathbb{R}$ with $g \uparrow_{P}=\bar{f} \uparrow_{P}=f$ and $g$ being nowhere-monotone on $\mathbb{R} \backslash P$. In other words, $g$ is a differentiable extension of $f$ with $g$ being nowhere-monotone on $\mathbb{R} \backslash P$.

To extend $\mathfrak{f}: \mathfrak{X} \rightarrow \mathfrak{X}$, note that $\mathfrak{X} \subseteq \mathfrak{C}$ is nowhere-dense and closed. It is clear that if the differentiable extension $g$ is nowhere-monotone on a dense open set $\mathbb{R} \backslash P$, then it is also nowheremonotone on $\mathbb{R}$. Theorem 1.4.3 immediately implies our desired extension.

Corollary 1.4.4. There exists a differentiable monster $g: \mathbb{R} \rightarrow \mathbb{R}$ extending the function $\mathfrak{f}: \mathfrak{X} \rightarrow \mathfrak{X}$ from (1.8).

\subsubsection{Weierstrass's monsters extending $\mathfrak{f}$}

Theorem 1.4.5. There exists a Weierstrass's monster $g: \mathbb{R} \rightarrow \mathbb{R}$ extending $\mathfrak{f}: \mathfrak{X} \rightarrow \mathfrak{X}$ from (1.8).

The construction is again based on Jarník's extension theorem and the following lemma.

Lemma 1.4.6. Let $f:[0,1] \rightarrow \mathbb{R}$ be non-constant, continuous, with $f(0)=0=f(1)$. Let $\mathcal{K}$ be the family of all connected components of $[0,1] \backslash \mathfrak{C}$. If $h:[0,1] \rightarrow \mathbb{R}$ is defined as

$$
h(x):= \begin{cases}(b-a) f\left(\frac{x-a}{b-a}\right) & \text { for } x \in(a, b) \in \mathcal{K} \\ 0 & \text { for } x \in \mathfrak{C}\end{cases}
$$

then $h$ is continuous but not differentiable at any $x \in \mathfrak{C}$.

Proof. For every $n \in \mathbb{N}$ let $\mathcal{K}_{n}:=\left\{(a, b) \in \mathcal{K}: b-a=\frac{1}{3^{n}}\right\}$ and $h_{n}:[0,1] \rightarrow \mathbb{R}$ be defined as

$$
h_{n}(x):= \begin{cases}(b-a) f\left(\frac{x-a}{b-a}\right) & \text { for } x \in(a, b) \in \mathcal{K}_{n} \\ 0 & \text { otherwise. }\end{cases}
$$

Then $h_{n}$ is continuous, $\left\|h_{n}\right\|_{\infty}=\frac{\|f\|_{\infty}}{3^{n}}$, and so $h=\sum_{n=1}^{\infty} h_{n}$ is continuous by Weierstrass M-test.

Since $f$ is non-constant, we have $M:=\|f\|_{\infty}>0$. Now fix an arbitrary $x \in \mathfrak{C}$. For every $n \in \mathbb{N}$, choose an $\left(a_{n}, b_{n}\right) \in \mathcal{K}_{n}$ closest to $x$ and an $x_{n} \in\left(a_{n}, b_{n}\right)$ with $\left|h_{n}\left(x_{n}\right)\right|=\left(b_{n}-a_{n}\right) M=\left|h\left(x_{n}\right)\right|$. Then, by the construction of $\mathfrak{C}, 0<\left|x_{n}-x\right|<2\left(b_{n}-a_{n}\right)=\frac{2}{3^{n}}$ so that $\lim _{n \rightarrow \infty} x_{n}=x$. Moreover,

$$
\left|\frac{h\left(x_{n}\right)-h(x)}{x_{n}-x}\right|=\frac{\left|h\left(x_{n}\right)\right|}{\left|x_{n}-x\right|}=\frac{\left(b_{n}-a_{n}\right) M}{\left|x_{n}-x\right|}>\frac{\left(b_{n}-a_{n}\right) M}{2\left(b_{n}-a_{n}\right)}=\frac{M}{2} .
$$


Thus the finite derivative $h^{\prime}(x)$ indeed does not exist.

Proof of Theorem 1.4.5. Let $f$ be the restriction of the Weierstrass's monster from $(1.1)$ to $[0,1]$ and notice that $f(0)=f(1)=0$. We use it to define an $h:[0,1] \rightarrow \mathbb{R}$ as in Lemma 1.4.6. Then $h$ is a Weierstrass's monster with $h \Upsilon_{\mathfrak{C}}=0$. It is easy to extend $h$ to a Weierstrass's monster $\tilde{h}$ on $\mathbb{R}$.

To construct $g$, let $\overline{\mathfrak{f}}: \mathbb{R} \rightarrow \mathbb{R}$ be a differentiable extension of $\mathfrak{f}$. Define $g:=\overline{\mathfrak{f}}+\tilde{h}$. Clearly, $g$ is continuous and, since $\mathfrak{X} \subseteq \mathfrak{C}$ and $\tilde{h} \uparrow_{\mathfrak{C}}=h \uparrow_{\mathfrak{C}}=0$, we also have $g\left\lceil_{\mathfrak{X}}=\overline{\mathfrak{f}} \uparrow_{\mathfrak{X}}+\tilde{h}\left\lceil_{\mathfrak{X}}=\mathfrak{f}\right.\right.$. Finally, $g$ cannot be differentiable at any $x \in \mathbb{R}$, since otherwise $\tilde{h}=g-\bar{f}$ would be.

\subsection{Typicality of differentiable extensions in $\mathrm{E}_{\Phi}(\mathbb{R})$}

Let $\Phi: P \rightarrow \mathbb{R}$ be a differentiable function defined on a closed set $P \subseteq \mathbb{R}$. As Theorem 1.4.3 has ensured, there exists a differentiable extension of $\Phi$ on $\mathbb{R}$ that is nowhere-monotone on $\mathbb{R} \backslash P$ and thus it is Pompeiu-like on every connected components of $\mathbb{R} \backslash P$. In what follows, let $\mathrm{E}_{\Phi}(\mathbb{R})$ be the family of all differentiable extensions of $\Phi$ which are Pompeiu-like on every connected components of $\mathbb{R} \backslash P$ and consider it with the uniform metric $\rho$ defined in Section 1.5.1. In this section, we show that $\mathrm{E}_{\Phi}(\mathbb{R})$, with $\rho$, is a complete metric space and that a typical function in it is nowhere-monotone on $\mathbb{R} \backslash P$.

Here we use the term typical to say that the set of all such functions is residual, that is, contains a dense $G_{\delta}$-set. Note that $P$ is not required to be nontrivial, that is, our study of differentiable extensions in Section 1.5 includes the case of extending an empty function. In such a case, we write $\mathrm{E}(\mathbb{R})$ as the collection of all Pompeiu-like functions on $\mathbb{R}$ equipped with the the same metric $\rho$.

\subsubsection{The space of differentiable extensions}

Let $\mathrm{D}(\mathbb{R})$ be the family of all differentiable functions from $\mathbb{R}$ into $\mathbb{R}$ and let $\mathrm{C}^{1}(\mathbb{R})$ stand for all $f \in \mathrm{D}(\mathbb{R})$ with derivative $f^{\prime}$ being continuous. It is well known that the subspace of $\mathrm{C}^{1}(\mathbb{R})$, consisting of all functions $f \in \mathrm{C}^{1}(\mathbb{R})$ for which their $\mathrm{C}^{1}$-norm

$$
\|f\|_{C^{1}}:=\|f\|_{\infty}+\left\|f^{\prime}\right\|_{\infty}
$$

is finite, forms a Banach space. ${ }^{31}$ Of course, $\|\cdot\|_{\infty}$ is the supremum norm.

\footnotetext{
${ }^{31}$ See [39, example 5.4]
} 
Proposition 1.5.1. If a sequence $\left\langle f_{n} \in \mathrm{D}(\mathbb{R}): n \in \mathbb{N}\right\rangle$ converges uniformly to an $f \in \mathrm{C}(\mathbb{R})$ and the sequence $\left\langle f_{n}^{\prime}: n \in \mathbb{N}\right\rangle$ is Cauchy with respect to the uniform convergence, then $f$ is differentiable and $\lim _{n \rightarrow \infty} f_{n}^{\prime}=f^{\prime}$.

Proposition 1.5.1 immediately implies also that:

Proposition 1.5.2. $\mathrm{D}(\mathbb{R})$ is a complete metric with respect to metric

$$
\rho(f, g):=\min \left\{1,\|f-g\|_{\mathrm{C}^{1}}\right\} .
$$

In what follows, $P$ will always denote a closed subset of $\mathbb{R}$ and $\Phi$ a differentiable function from $P$ into $\mathbb{R}$. For $f \in \mathrm{D}(\mathbb{R})$, we define

$$
\begin{aligned}
& \mathrm{D}_{\Phi}(\mathbb{R}):=\{f \in \mathrm{D}(\mathbb{R}): f \supseteq \Phi\} \\
& \mathrm{E}_{\Phi}(\mathbb{R}):=\left\{f \in \mathrm{D}(\mathbb{R}): f \supseteq \Phi \&\left[f^{\prime}=0\right] \text { is dense in } \mathbb{R} \backslash P\right\}
\end{aligned}
$$

Lemma 1.5.3. $\mathrm{E}_{\Phi}(\mathbb{R})$ is a nonempty closed subspace of $\mathrm{D}(\mathbb{R})$ considered with the metric $\rho$. In particular, $\mathrm{E}_{\Phi}(\mathbb{R})$ is a complete metric space.

Proof. It has been proved in Theorem 1.4.3 that there is an $f \in \mathrm{D}_{\Phi}(\mathbb{R})$ which is nowhere-monotone on $\mathbb{R} \backslash P$. Such $f$ belongs to $\mathrm{E}_{\Phi}(\mathbb{R})$ since the derivative $f^{\prime}$ must satisfy (1.3). This shows that $\mathrm{E}_{\Phi}(\mathbb{R})$ is nonempty. Clearly, $D_{\Phi}(\mathbb{R})$ is closed in $\mathrm{D}(\mathbb{R})$. Since $\mathrm{E}_{\Phi}(\mathbb{R})=\mathrm{D}_{\Phi}(\mathbb{R}) \cap \mathrm{E}(\mathbb{R})$, it remains to show that $\mathrm{E}(\mathbb{R})$ is closed in $\mathrm{D}(\mathbb{R})$. Our argument for this comes from a paper [79] of C. Weil.

To see this, assume that a sequence $\left\langle f_{n} \in \mathrm{E}(\mathbb{R}): n \in \mathbb{N}\right\rangle$ converges to an $f \in \mathrm{D}(\mathbb{R})$ with respect to $\rho$. We need to show that $f \in \mathrm{E}(\mathbb{R})$. Indeed, for every $n \in \mathbb{N}$, the set $G_{n}:=\left[f_{n}^{\prime}=0\right]$ is dense in $\mathbb{R}$. It is also $G_{\delta}$ in $\mathbb{R}$ since a derivative is of Baire class one. ${ }^{32}$ Hence $f^{\prime}=\lim _{n \rightarrow \infty} f_{n}^{\prime}$ has value 0 on the set $\bigcap_{n \in \mathbb{N}} G_{n}$, which is also dense $G_{\delta}$ in $\mathbb{R}$ as $\mathbb{R}$ is a Baire space.

Notice that we proved also that $\mathrm{E}(\mathbb{R})$ is nonempty and closed in $\mathrm{D}(\mathbb{R})$.

Remark 1.5.4. If $P=\operatorname{dom}(\Phi) \neq \mathbb{R}$, then the space $\mathrm{E}_{\Phi}(\mathbb{R})$, with the metric $\rho$, has a closed discrete subset of cardinality continuum. In particular, neither $\mathrm{E}_{\Phi}(\mathbb{R})$ nor $\mathrm{D}(\mathbb{R})$ is separable.

Proof. Let $a<b$ be such that $[a, b] \cap P=\emptyset$ and let $f \in \mathrm{E}_{\Phi}(\mathbb{R})$. Choose a sequence $b=b_{1}>$ $a_{1}>b_{2}>a_{2}>\cdots$ converging to $a$ such that $a$ is a Lebesgue density point of the complement of $\bigcup_{n \in \mathbb{N}}\left(a_{n}, b_{n}\right)$. For every $n \in \mathbb{N}$, let $g_{n}: \mathbb{R} \rightarrow \mathbb{R}$ be a map as in Lemma 1.5.6 with support in ${ }^{32}$ See [26, theorem 2.2]. 
$\left[a_{n}, b_{n}\right]$ and vertically rescaled so that $\left\|g_{n}^{\prime}\right\|_{\infty}=1$. For every $s: \mathbb{N} \rightarrow\{-1,1\}$, let $h_{s}=\sum_{n \in \mathbb{N}} s(n) g_{n}^{\prime}$. It is bounded and approximately continuous - this is ensured at $x=a$ by the Lebesgue density requirement.

This implies that each $H_{s}(x)=\int_{0}^{x} h_{s}(t) d t$ is in $\mathrm{E}(\mathbb{R})$, since $H_{s}^{\prime}=h_{s} \cdot{ }^{33}$ In particular, $H_{s}+f \in$ $\mathrm{E}_{\varphi}(\mathbb{R})$. Also, for every distinct $s, t: \mathbb{N} \rightarrow\{-1,1\}$, we have $\rho\left(H_{s}+f, H_{t}+f\right)=\min \left\{1,\left\|H_{s}-H_{t}\right\|_{\mathrm{C}^{1}}\right\} \geq$ $\min \left\{1,\left\|H_{s}^{\prime}-H_{t}^{\prime}\right\|_{\infty}\right\}=1$. That is, we indeed found a closed discrete subset $\left\{H_{s}+f: s: \mathbb{N} \rightarrow\{-1,1\}\right\}$ of $\mathrm{E}_{\Phi}(\mathbb{R})$ of cardinality continuum.

\subsubsection{Differentiable extensions that are nowhere-monotone}

Theorem 1.5.5. If $P \subseteq \mathbb{R}$ is closed and $\Phi: P \rightarrow \mathbb{R}$ is differentiable, then a typical function in $\mathrm{E}_{\Phi}(\mathbb{R})$ is nowhere-monotone on $\mathbb{R}$.

The key step in the proof of Theorem 1.5.5 is the following lemma, which is based on Proposition 1.3.6.

Lemma 1.5.6. For every $a<b<c<d$, there exists a "bump" map $g \in \mathrm{E}(\mathbb{R})$ strictly increasing on $(a, c)$, strictly decreasing on $(c, d)$ such that $g^{\prime}(b)>0,\|g\|_{\mathrm{C}^{1}}<\infty$, and with $g(x)=0$ for any $x \in \mathbb{R} \backslash(a, d)$.

Proof. Let $\varphi:[0,1] \rightarrow[0,1]$ a strictly increasing P-filling from Proposition 1.3.6. Pick a $t \in(0,1)$ such that $\varphi^{\prime}(t)>0$ and let $\tilde{\varphi}:=\varphi\left\lceil_{[0, t]}\right.$. Let $\mathrm{L}_{1}:[a, b] \rightarrow[0, t]$ be an increasing linear surjection, and $\mathrm{L}_{2}:[b, c] \rightarrow[0, t]$ and $\mathrm{L}_{3}:[c, d] \rightarrow[0,1]$ be two decreasing linear surjections. Then

$$
g(x):= \begin{cases}\left(\tilde{\varphi} \circ \mathrm{L}_{1}\right)(x) & \text { for } x \in[a, b] \\ \tilde{\varphi}(t)+\frac{c-b}{b-a}\left(\tilde{\varphi}(t)-\left(\tilde{\varphi} \circ \mathrm{L}_{2}\right)(x)\right) & \text { for } x \in(b, c) \\ \left(\tilde{\varphi}(t)+\frac{c-b}{b-a} \tilde{\varphi}(t)\right)\left(\varphi \circ \mathrm{L}_{3}\right)(x) & \text { for } x \in[c, d] \\ 0 & \text { for } x \in \mathbb{R} \backslash[a, d]\end{cases}
$$

is as needed.

Proof of Theorem 1.5.5. For an open nonempty interval $I \subseteq \mathbb{R} \backslash P$, let

$$
U_{I}^{+}:=\left\{f \in \mathrm{E}_{\Phi}(\mathbb{R}):(\exists x \in I) f^{\prime}(x)>0\right\}
$$

\footnotetext{
${ }^{33}$ See [12, theorem 7.38].
} 
and

$$
U_{I}^{-}:=\left\{f \in \mathrm{E}_{\Phi}(\mathbb{R}):(\exists x \in I) f^{\prime}(x)<0\right\} .
$$

We claim that the sets $U_{I}^{+}$and $U_{I}^{-}$are open and dense in $\mathrm{E}_{\Phi}(\mathbb{R})$.

Indeed, they are open, since for every $f \in U_{I}^{-}$(or $f \in U_{I}^{+}$) and $x \in I$ for which $f^{\prime}(x)<0$ $\left(f^{\prime}(x)>0\right.$, respectively), the $\rho$-ball centered at $f$ and of radius $\left|f^{\prime}(x)\right|$ is contained in $U_{I}^{-}\left(U_{I}^{+}\right.$, respectively).

To see that $U_{I}^{+} \cap U_{I}^{-}$is dense in $\mathrm{E}_{\Phi}(\mathbb{R})$, choose an arbitrary $f \in \mathrm{E}_{\Phi}(\mathbb{R})$ and $\varepsilon \in(0,1)$. It is enough to find a $g \in \mathrm{D}(\mathbb{R})$ with $\|g\|_{\mathrm{C}^{1}}<\varepsilon$ such that $f+g \in U_{I}^{+} \cap U_{I}^{-}$. To find such a $g$ choose $a_{0}<b_{0}<d_{0}<a_{1}<b_{1}<d_{1}$ in $I$ with $f^{\prime}$ equal 0 at each of these points. Let $g_{0}$ and $g_{1}$ be as in Lemma 1.5.6 applied to numbers $a_{0}<b_{0}<d_{0}$ and $a_{1}<b_{1}<d_{1}$, respectively. Multiplying these functions by a sufficiently small constant if necessary, we can further assume that $\left\|g_{0}\right\|_{\mathrm{C}^{1}}<\varepsilon$ and $\left\|g_{1}\right\|_{\mathrm{C}^{1}}<\varepsilon$. Then the function $g:=g_{0}-g_{1}$ is as needed.

Finally, let $\mathcal{I}$ be the countable family of all nonempty intervals $I \subseteq \mathbb{R} \backslash P$ with rational endpoints. Then $G:=\bigcap_{I \in \mathcal{I}}\left(U_{I}^{+} \cap U_{I}^{-}\right)$is a dense $G_{\delta}$ set in $\mathrm{E}_{\Phi}(\mathbb{R})$, and every function in $G$ is nowhere-monotone on $\mathbb{R}$.

\subsubsection{Differentiable extensions that are nowhere nowhere-monotone}

We say that a function $f: \mathbb{R} \rightarrow \mathbb{R}$ is increasing at a point $x \in \mathbb{R}$ provided there exists an interval $(a, b)$ containing $x$ on which $f$ is strictly increasing. Let $\mathrm{M}_{\Phi}^{\nearrow}(\mathbb{R})$ be the family of all $f \in \mathrm{E}_{\Phi}(\mathbb{R})$ for which the set of points at which $f$ is increasing is dense in $\mathbb{R} \backslash P$.

Similarly, we say that $f: \mathbb{R} \rightarrow \mathbb{R}$ is monotone (constant or decreasing) at a point $x \in \mathbb{R}$ provided there exists an interval $(a, b)$ containing $x$ on which $f$ is monotone, constant, or strictly decreasing, respectively. With each of these notions we associate their respective families $M_{\Phi}(\mathbb{R}), M_{\Phi}(\mathbb{R})$, and $\mathrm{M}_{\Phi}^{\searrow}(\mathbb{R})$, in a way in which $\mathrm{M}_{\Phi}^{\nearrow}(\mathbb{R})$ is associated with the notion of "increasing at a point."

Clearly, $\mathrm{M}_{\Phi}^{\nearrow}(\mathbb{R}), \mathrm{M}_{\Phi}^{\overrightarrow{ }}(\mathbb{R})$, and $\mathrm{M}_{\Phi}^{\searrow}(\mathbb{R})$ are disjoint and contained in $\mathrm{M}_{\Phi}(\mathbb{R})$ which, by Theorem 1.5.5, is first category in $\mathrm{E}_{\Phi}(\mathbb{R})$. The goal of this section is to show that each of these first category sets is dense in $\mathrm{E}_{\Phi}(\mathbb{R})$.

Theorem 1.5.7. Each of the sets $\mathrm{M}_{\Phi}^{\lambda}(\mathbb{R}), \mathrm{M}_{\Phi}^{\rightarrow}(\mathbb{R})$, and $\mathrm{M}_{\Phi}^{\searrow}(\mathbb{R})$ is dense in $\mathrm{E}_{\Phi}(\mathbb{R})$.

The main step in the proof of Theorem 1.5.7 is the following Lemma 1.5.8.

Lemma 1.5.8. For every $f \in \mathrm{E}_{\Phi}(\mathbb{R}), \varepsilon \in(0,1)$, and a nonempty open set $U \subseteq \mathbb{R} \backslash P$, there exist $f_{1} \in \mathrm{E}_{\Phi}(\mathbb{R})$ and $p<q<r<s$ with $(p, s) \subseteq U$ such that $f_{1}=f$ on $\mathbb{R} \backslash(p, s), \rho\left(f_{1}, f\right)<\varepsilon, f_{1} \Upsilon_{(p, q)}$ 
is strictly increasing, $f_{1} \Upsilon_{(q, r)}$ is constant, and $f_{1} \Upsilon_{(r, s)}$ is strictly decreasing.

Proof. Let $g$ be as in Lemma 1.5.6 with $a=0, c=1$, and $d=2$. Multiplying it by a sufficiently small constant if necessary, we can further assume that $g(c)=1$. Let $M:=\|g\|_{\mathrm{C}^{1}}$ and notice that $M \geq 1$. Notice that for every $\delta>0$ the set $\left[\left|f^{\prime}\right| \geq \delta\right]$ cannot be dense in $U$ according to Proposition 1.3.3.

Let $\delta:=\frac{\varepsilon}{12 M}$ and choose a nonempty interval $(p, s) \subseteq U$ disjoint from $\left[\left|f^{\prime}\right| \geq \delta\right]$ and with $s-p \leq 1$. Decreasing this interval, if necessary, we can also assume that $f^{\prime}(p)=f^{\prime}(s)=0$ and that $|f(x)-f(y)| \leq \varepsilon / 4$ for every $x, y \in[p, s]$. Let $[q, r]$ be the middle third of $[p, s]$.

For some $\xi \in(p, s)$, we have $\left|\frac{f(s)-f(p)}{q-p}\right|=3\left|\frac{f(s)-f(p)}{s-p}\right|=3\left|f^{\prime}(\xi)\right|<3 \delta$. Thus, there exists a $v>\max \{f(s), f(p)\}$ such that $\frac{v-f(p)}{q-p}<3 \delta$ and $\frac{v-f(s)}{s-r}<3 \delta$. Consider the following two linear surjections: increasing $\mathrm{L}_{1}:[p, q] \rightarrow[0,1]$ and decreasing $\mathrm{L}_{2}:[r, s] \rightarrow[0,1]$. Notice, that $\mathrm{L}_{1}$ has the slope $m:=\frac{1}{q-p}$, while $\mathrm{L}_{2}$ has the slope $\frac{1}{s-r}=-m$. Define $g_{1}:=(v-f(p)) \cdot g \circ \mathrm{L}_{1}$ and notice that we have $g_{1}(p)=0$ and $g_{1}(q)=v-f(p)$. Also, $\left\|g_{1}^{\prime}\right\|_{\infty}=(v-f(p)) \frac{1}{q-p}\left\|g^{\prime}\right\|_{\infty} \leq \frac{v-f(p)}{q-p} M<3 \delta M=\varepsilon / 4$ and $\left\|g_{1}\right\|_{\infty}=(v-f(p))\|g\|_{\infty} \leq \frac{v-f(p)}{q-p} M<\varepsilon / 4$. Therefore, we have $\left\|g_{1}\right\|_{\mathrm{C}^{1}}=\left\|g_{1}\right\|_{\infty}+\left\|g_{1}^{\prime}\right\|_{\infty}<\varepsilon / 2$. Similarly, if $g_{2}:=(v-f(s)) \cdot g \circ \mathrm{L}_{2}$, then $\left\|g_{2}\right\|_{\mathrm{C}^{1}}<\varepsilon / 2, g_{2}(s)=0$, and $g_{2}(r)=v-f(s)$. Define

$$
f_{1}(x):= \begin{cases}f(p)+g_{1}(x) & \text { for } x \in[p, q] \\ v & \text { for } x \in[q, r] \\ f(s)+g_{2}(x) & \text { for } x \in[r, s] \\ f(x) & \text { for } x \in \mathbb{R} \backslash(p, s) .\end{cases}
$$

and notice that it is as needed.

Indeed, it is easy to see that $f_{1}$ is a well defined differentiable function with $f_{1}^{\prime}(p)=f_{1}^{\prime}(q)=$ $f_{1}^{\prime}(r)=f_{1}^{\prime}(s)=0$. All other requirements for $f_{1}$ are clearly satisfied, except possibly for $\rho\left(f_{1}, f\right)<\varepsilon$. To see this, it is enough to prove that $\left\|\left(f_{1}-f\right) \uparrow_{J}\right\|_{\mathrm{C}^{1}}<\varepsilon$ for $J$ being $[p, q],[q, r]$, and $[r, s]$.

But on $J=[p, q]$ we have

$$
\begin{aligned}
\left\|f_{1}-f\right\|_{\mathrm{C}^{1}} & \leq\left\|g_{1}\right\|_{\mathrm{C}^{1}}+\|f-f(p)\|_{\mathrm{C}^{1}} \\
& <\varepsilon / 2+\|f-f(p)\|_{\infty}+\left\|(f-f(p))^{\prime}\right\|_{\infty} \\
& <\varepsilon / 2+\varepsilon / 4+\delta<\varepsilon
\end{aligned}
$$


Similarly, $\left\|f_{1}-f\right\|_{\mathrm{C}^{1}}<\varepsilon$ on $J=[r, s]$. Finally, on $J=[q, r]$,

$$
\left\|f_{1}-f\right\|_{\mathrm{C}^{1}} \leq\|v-f(p)\|_{\mathrm{C}^{1}}+\|f-f(p)\|_{\mathrm{C}^{1}} \leq \varepsilon / 4+\varepsilon / 4+\delta<\varepsilon
$$

as needed.

Proof of Theorem 1.5.7. We prove only the density of $\mathrm{M}_{\Phi}^{X}(\mathbb{R})$, the proof for the other two cases being essentially the same. Fix an $f_{0} \in \mathrm{E}_{\Phi}(\mathbb{R})$ and an $\varepsilon \in(0,1)$. We need to find $g \in \mathrm{M}_{\Phi}^{\nearrow}(\mathbb{R})$ with $\left\|f_{0}-g\right\|_{\mathrm{C}^{1}} \leq \varepsilon$.

Let $\left\{B_{n}: n \in \mathbb{N}\right\}$ be the intervals forming a basis for $\mathbb{R} \backslash P$. Define $J_{0}=\emptyset$. By induction on $n \in \mathbb{N}$ we will construct the sequences $\left\langle J_{n} \subseteq \mathbb{R} \backslash P: n \in \mathbb{N}\right\rangle$ of pairwise disjoint, possibly empty, open intervals and $\left\langle f_{n} \in \mathrm{E}_{\Phi}(\mathbb{R}): n \in \mathbb{N}\right\rangle$ such that the following inductive properties hold for every $n \in \mathbb{N}$.

(i) $B_{n} \cap \bigcup_{i \leq n} J_{i} \neq \emptyset$,

(ii) $f_{n}$ is strictly increasing on each $J_{i}$ with $i \leq n, f_{n-1}=f_{n}$ on $\mathbb{R} \backslash \bigcup_{i<n} J_{i}$, and $\rho\left(f_{n-1}, f_{n}\right)<2^{-n} \varepsilon$.

The inductive step is facilitated by Lemma 1.5.8. Specifically, we let $U$ to be the interior of $B_{n} \backslash \bigcup_{i<n} J_{i}$. If $U=\emptyset$, we let $f_{n}=f_{n-1}$ and $J_{n}=\emptyset$. Otherwise we choose $p<q<r<s$ and $f_{n}$ by applying Lemma 1.5 .8 to $f_{n-1} \in \mathrm{E}_{\Phi}^{1}(\mathbb{R}), 2^{-n} \varepsilon>0$, and just chosen $U$. Let $J_{n}=(p, q)$. This choice of $f_{n}$ and $J_{n}$ ensures properties (i) and (ii).

To finish the proof, notice that the sequence $\left\langle f_{n} \in \mathrm{E}_{\Phi}(\mathbb{R}): n \in \mathbb{N}\right\rangle$ is Cauchy with respect to $\rho$, so that the limit $g=\lim _{n \rightarrow \infty} f_{n}$ exists and belongs to $\mathrm{E}_{\Phi}(\mathbb{R})$. By (ii), $\rho\left(f_{0}, f_{n}\right)<\left(1-2^{-n}\right) \varepsilon$ for every $n \in \mathbb{N}$. Therefore, $\rho\left(f_{0}, g\right) \leq \varepsilon$.

Finally, to see that $g \in \mathrm{M}_{\Phi}^{\nearrow}(\mathbb{R})$ notice that, by (i), for every $n \in \mathbb{N}$ there exists an $i \leq n$ such that $B_{n} \cap J_{i} \neq \emptyset$. Moreover, by (ii), the restriction $g \uparrow_{\left(B_{n} \cap J_{i}\right)}=f_{i} \uparrow_{\left(B_{n} \cap J_{i}\right)}$ is strictly increasing. So, indeed $g \in \mathrm{M}_{\Phi}^{\nearrow}(\mathbb{R})$. 


\section{Chapter 2}

\section{Paradoxes in Continuity}

The content of this chapter comes from a published article [23] which studied the Sierpiński-Zygmund functions in the classes of Darboux-like functions. ${ }^{1}$ Seven among the nine examples constructed below have been unknown before the publication of [23], and they constitute the answers to the problems posted in the preliminary version of a survey [27, theorem 4.10] of K. Ciesielski and J. Seoane-Sepúlveda. The existence of these examples has caused some studies in the lineability of Sierpiński-Zygmund functions in different Darboux-like classes. ${ }^{2}$

\section{$2.1 \quad$ Introduction}

For $X \subseteq \mathbb{R}$, a map $f: X \rightarrow \mathbb{R}$ is a Sierpiński-Zygmund function (or just SZ-function) provided $f\left\lceil_{S}\right.$, its restriction to $S$, is discontinuous for any $S \subseteq X$ of cardinality $\mathfrak{c}$. Here $\mathfrak{c}$ stands for the continuum, that is, the cardinality of $\mathbb{R}$. The first example of such function $f: \mathbb{R} \rightarrow \mathbb{R}$ was constructed in a 1923 paper [72] of Wacław Sierpiński and Antoni Zygmund. The SZ-maps have "as little continuity as possible."

On the other hand, an $f: \mathbb{R} \rightarrow \mathbb{R}$ is Darboux provided it satisfies the intermediate value property, that is, for every $a<b$ and $y$ between $f(a)$ and $f(b)$, there is a $c \in[a, b]$ with $f(c)=y$. This is equivalent to the fact that $f[C]$ is connected (i.e., an interval) for every connected $C \subseteq \mathbb{R}$. The name is used in honor of Jean Gaston Darboux who, in a 1875 paper [28], has shown that all derivatives, including those that are discontinuous, have the intermediate value property. The

\footnotetext{
${ }^{1}$ Reprinted by permission from Springer Nature Customer Service Centre GmbH: Springer Nature, Banach Journal of Mathematical Analysis, Examples of Sierpiński-Zygmund maps in the class of Darboux-like functions, Krzysztof Chris Ciesielski \& Cheng-Han Pan], 2020.

${ }^{2}$ See e.g., [1]
} 
classes of Sierpiński-Zygmund and Darboux functions from $\mathbb{R}$ to $\mathbb{R}$ are denoted, respectively, by the symbols SZ and $\mathcal{D}$.

By definition, any $f \in \mathcal{D}$ shares the intermediate value property with the class of all continuous functions from $\mathbb{R}$ to $\mathbb{R}$. As such, $\mathcal{D}$ can be considered as a class of generalized continuous functions. Since SZ contains only extremely discontinuous functions, it is not surprising that SZ contains no generalized continuous function in the most typical uses of such term, including, but not limited to, approximately or $\mathcal{I}$-approximately continuous functions and Borel, Baire, or Lebesgue measurable functions. But this is where the class $\mathcal{D}$ stands apart from the other classes of generalized continuous functions in a 1997 paper [6], M. Balcerzak, K. Ciesielski and T. Natkaniec proved that existence of Darboux SZ-functions is independent of ZFC, the standard axioms of set theory. ${ }^{3}$ More precisely, it has been proven in [6] that $\mathrm{SZ} \cap \mathcal{D}=\emptyset$ in the iterated perfect set model and constructed an $f \in \mathrm{SZ} \cap \mathcal{D}$ under the assumption that $\operatorname{cov}_{\mathrm{M}}=\mathfrak{c}$, where

$$
\operatorname{cov}_{\mathrm{M}}:=\{\kappa: \mathbb{R} \text { is not a union of less than } \kappa \text {-many meager sets in } \mathbb{R}\}
$$

Notice that the property $\operatorname{cov}_{M}=\mathfrak{c}$ is consistent with ZFC, as it follows from the Continuum Hypothesis. ${ }^{4}$ For more details concerning this discussion, see [27].

All classes of Darboux-like functions we discuss in this paper are contained in $\mathcal{D}$. Therefore, all our constructions require an additional set-theoretical assumption, which we keep as in [6], that is, we use the assumption $\operatorname{cov}_{M}=\mathfrak{c}: \mathbb{R}$ is not a union of less than $\mathfrak{c}$-many meager sets. More specifically, we will use the following well-known and easy-to-see result.

Proposition 2.1.1. If $\operatorname{cov}_{\mathrm{M}}=\mathfrak{c}$ holds, then no $G_{\delta}$-subset of $\mathbb{R}$ which is dense in some nontrivial interval is a union of less than $\mathfrak{c}$-many meager subsets of $\mathbb{R}$.

Let $\mathcal{G}=\left\{f \in \mathbb{R}^{G}: f\right.$ is continuous and $G$ is a $G_{\delta^{-}}$-subset of $\left.\mathbb{R}\right\}$ and note that $|\mathcal{G}|=\mathrm{c}^{5}$ A standard construction, including the original one from the 1923 paper of Wacław Sierpiński and Antoni Zygmund, uses the following result of K. Kuratowski. ${ }^{6}$

Proposition 2.1.2 (Kuratowski). For every continuous function $g$ from $S \subseteq \mathbb{R}$ to $\mathbb{R}$, there exists a $G_{\delta}$-set $G \subseteq \mathbb{R}$ containing $S$ and a continuous extension $\bar{g}: G \rightarrow \mathbb{R}$ of $g$.

\footnotetext{
${ }^{3}$ This result settled a problem posed in a 1993 paper [29] by U. Darji, who constructed there, in ZFC, a function in $\mathrm{SZ} \cap \mathrm{PR}$ and asked about a function in $\mathrm{SZ} \cap \mathcal{D}$. For more on this subject, see survey [27].

${ }^{4}$ More generally, from the Martin's Axiom.

${ }^{5}$ Since a continuous function is uniquely determined by its values on a dense set and the fact that there are $\mathfrak{c}-$ many $G_{\delta}$ subsets of $\mathbb{R}$, a simple cardinality argument gives $|\mathcal{G}|=\mathfrak{c} \otimes \mathfrak{c}^{\omega}=\mathfrak{c}$.

${ }^{6}$ See e.g., [49, theorem 3.8, page 16$]$.
} 
In what follows, we will repeatedly use the following well-known result that follows immediately from Proposition 2.1.2. In its statement, and in what follows, we identify any function with its graph.

Proposition 2.1.3. If $f$ is a function from $X \subseteq \mathbb{R}$ into $\mathbb{R}$ such that $|f \cap g|<\mathfrak{c}$ for every $g \in \mathcal{G}$, then $f \in \mathrm{SZ}$.

Other simple and well-known properties of Sierpiński-Zygmund functions that we will use are as follows.

Proposition 2.1.4. (i) Any restriction of a Sierpiński-Zygmund function is Sierpiński-Zygmund.

(ii) If $f: \mathbb{R} \rightarrow \mathbb{R}$ is a union of countably many Sierpinski-Zygmund functions, then $f \in \mathrm{SZ}$.

In the standard setting, the collection of Darboux-like classes of functions from $\mathbb{R}$ to $\mathbb{R}$ encompasses, beside of the class $\mathcal{D}$, also seven other classes: $\mathrm{PC}$ of peripherally continuous functions, PR of functions with perfect road, Conn of connectivity functions, AC of almost continuous functions, Ext of extendable functions, CIVP of functions with Cantor intermediate value property, and SCIVP of functions with strong Cantor intermediate value property. We will provide their definitions in the following sections on the as needed basis. The inclusion relations among them are presented in Fig. 2.1.

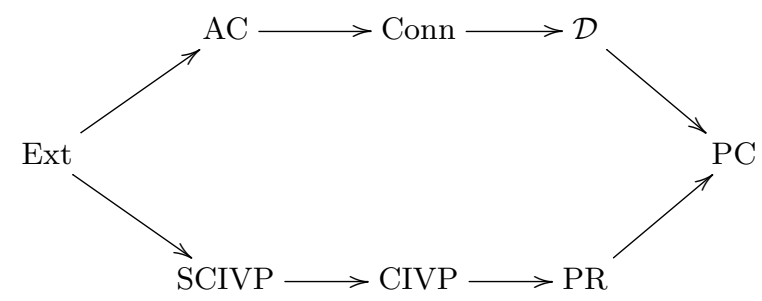

Figure 2.1: All inclusions, indicated by arrows, among the Darboux-like classes of functions from $\mathbb{R}$ to $\mathbb{R}$. The only inclusions among the intersections of these classes are those that follows trivially from this schema. (See [35], [19], or [27].)

It is worthy to mention that while Fig. 2.1 remains unchanged when we restrict Darboux-like classes to Baire class two functions, all classes represented there coincide ${ }^{7}$ when restricted to the class of Baire one functions. ${ }^{8}$ Also, directly by the definition (not provided here), any SCIVP function has continuous restrictions to many perfect sets. Thus, SZ $\cap$ SCIVP $=\emptyset$ and, by Fig. 2.1, also $\mathrm{SZ} \cap \mathrm{Ext}=\emptyset$. In particular, in what follows we will be interested only in the classes in Fig. 2.2.

\footnotetext{
${ }^{7}$ I.e., are equal.

${ }^{8}$ See the references in [19] or [27].
} 


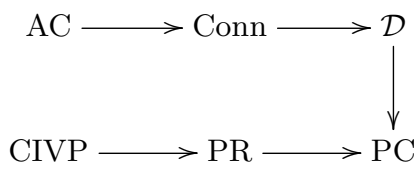

Figure 2.2: Six Darboux-like classes of functions that consistently contain SZ-functions. Arrows indicate strict inclusions.

Notice that the algebra of subsets of $\mathcal{D}$ generated by the classes in Fig. 2.2 has 9 atoms: $\mathcal{D} \backslash(\mathrm{Conn} \cup \mathrm{PR}), \quad \mathcal{D} \cap \mathrm{PR} \backslash(\mathrm{Conn} \cup \mathrm{CIVP}), \quad \mathcal{D} \cap \mathrm{CIVP} \backslash$ Conn,$\quad$ Conn $\backslash(\mathrm{AC} \cup \mathrm{PR})$, Conn $\cap \mathrm{PR} \backslash(\mathrm{AC} \cup \mathrm{CIVP})$, Conn $\cap \mathrm{CIVP} \backslash \mathrm{AC}, \mathrm{AC} \backslash \mathrm{PR}, \mathrm{AC} \cap \mathrm{PR} \backslash \mathrm{CIVP}$, and $\mathrm{AC} \cap \mathrm{CIVP}$. The algebra of subsets of $\mathrm{PC} \backslash \mathcal{D}$ generated by the classes in Fig. 2.2 has only 3 atoms, and it can be proved in ZFC that they have a non-empty intersections with SZ. ${ }^{9}$

The main goal of this chapter is to construct, under the additional set-theoretical assumption that $\operatorname{cov}_{\mathrm{M}}=\mathfrak{c}$, the examples of functions belonging to the following nine classes, the intersections of the above-mentioned atoms with SZ:

(i) $\mathrm{SZ} \cap \mathcal{D} \backslash(\mathrm{Conn} \cup \mathrm{PR})$;

(ii) $\mathrm{SZ} \cap \mathcal{D} \cap \mathrm{PR} \backslash(\mathrm{Conn} \cup \mathrm{CIVP})$;

(iii) $\mathrm{SZ} \cap \mathcal{D} \cap \mathrm{CIVP} \backslash \mathrm{Conn}$;

(iv) $\mathrm{SZ} \cap \mathrm{Conn} \backslash(\mathrm{AC} \cup \mathrm{PR})$;

(v) $\mathrm{SZ} \cap \mathrm{Conn} \cap \mathrm{PR} \backslash(\mathrm{AC} \cup \mathrm{CIVP})$;

(vi) $\mathrm{SZ} \cap \mathrm{Conn} \cap \mathrm{CIVP} \backslash \mathrm{AC}$;

(vii) $\mathrm{SZ} \cap \mathrm{AC} \backslash \mathrm{PR}$;

(viii) $\mathrm{SZ} \cap \mathrm{AC} \cap \mathrm{PR} \backslash \mathrm{CIVP}$;

(ix) $\mathrm{SZ} \cap \mathrm{AC} \cap \mathrm{CIVP}$;

Notice that for the first seven of these classes, the existence of such examples was previously unknown, and their construction solves $[27$, problems $4.10,4.13,4.14]$. We also show that the machinery we construct here allows also easy constructions of the remaining two previously-known examples.

${ }^{9}$ See e.g., [27]. 


\subsection{Examples of Sierpiński-Zygmund functions in the class of Darboux-like functions}

\subsection{1 $\mathrm{SZ} \cap \mathrm{AC} \backslash \mathrm{PR}$}

Under the Continuum Hypothesis, $\mathrm{SZ} \cap \mathrm{AC} \neq \emptyset$ was first noticed in a 1982 paper [50] of K. R. Kellum, who pointed out that a function in SZ $\cap$ Conn constructed by J. Ceder in his 1981 paper [13] is also AC. An example of a function in $\mathrm{SZ} \cap \mathrm{AC} \cap \mathrm{PR}$ was constructed, under $\operatorname{cov}_{\mathrm{M}}=\mathfrak{c}$, in a 1997 paper [6] of M. Balcerzak, K. Ciesielski, and T. Natkaniec.

Definition 2.2.1. A function $f: \mathbb{R} \rightarrow \mathbb{R}$ has a perfect road at every $x \in \mathbb{R}$, denoted as $f \in \mathrm{PR}$, provided for every $x \in \mathbb{R}$, there exists a perfect set $P \subseteq \mathbb{R}$ having $x$ as a bilateral limit point such that $f \uparrow_{P}$ is continuous at $x$.

This class was first introduced in a 1936 paper [58] of I. Maximoff, where he proved that a Baire class one function is Darboux if, and only if, it has a perfect road at each point.

In what follows, we will use the following simple fact.

Proposition 2.2.2. If $f: \mathbb{R} \rightarrow \mathbb{R}$ is unbounded on every perfect subset of $\mathbb{R}$, then $f$ has perfect road at no point.

Proof. Suppose $f$ has a perfect road at $x \in \mathbb{R}$. Then, there exists a perfect $P$ having $x$ as a bilateral limit point such that $f \uparrow_{P}$ is continuous at $x$. By continuity, there exists an $\delta>0$ such that $f \uparrow_{P}$ is bounded on $P \cap[x-\delta, x+\delta]$. However, $P \cap[x-\delta, x+\delta]$ contains a perfect set, a contradiction.

Definition 2.2.3. A function $f: \mathbb{R} \rightarrow \mathbb{R}$ is almost continuous, denoted as $f \in \mathrm{AC}$, provided every open set in $\mathbb{R}^{2}$ containing the graph of $f$ contains also the graph of a continuous function from $\mathbb{R}$ to $\mathbb{R}$.

This class was first seriously studied in a 1959 paper [73] of J. Stallings. However it appeared already in an earlier paper [37] of O. H. Hamilton.

The above definition emphasizes the similarities between continuous and almost continuous functions. However, a more useful characterization of functions in $\mathrm{AC}$ relies on the notion of blocking sets. Specifically, a $B \subseteq \mathbb{R}^{2}$ is a blocking set provided it is closed, meets the graph of every continuous function, and is disjoint with some arbitrary function $h \in \mathbb{R}^{\mathbb{R}}$. In what follows, the family of all 
blocking sets will be denoted by $\mathbb{B}$. It is an easy exercise to see that

$$
\text { a function } \bar{f}: \mathbb{R} \rightarrow \mathbb{R} \text { is } \mathrm{AC} \text { if, and only if, } \bar{f} \cap B \neq \emptyset \text { for every } B \in \mathbb{B} .^{10}
$$

More interestingly, every $\hat{B} \in \mathbb{B}$ contains another blocking set $B$ such that $\pi[B]$ is a nontrivial interval, ${ }^{11}$ where $\pi[B]$ is the projection of $B$ onto the first coordinate. ${ }^{12}$ In particular, if

$$
\mathbb{K}:=\left\{K \subseteq \mathbb{R}^{2}: K \text { is compact and } \pi[K]=[a, b] \text { for some } a<b\right\},
$$

then we have the following easy and well-known result.

Proposition 2.2.4. If $f \in \mathbb{R}^{\mathbb{R}}$ is such that $f \cap K \neq \emptyset$ for every $K \in \mathbb{K}$, then $f \in \mathrm{AC}$.

Proof. By (2.1), it is enough to show that every $B \in \mathbb{B}$ contains some $K \in \mathbb{K}$. But, by the above discussion, $\pi[B]$ has a non-empty interior. Since $B$ is a countable union of compact sets $B_{n}, n<\omega$, and $\pi[B]$ is a union of compact sets $\pi\left[B_{n}\right]$, by Baire category theorem there exists an $n<\omega$ such that $\pi\left[B_{n}\right]$ has a non-empty interior. Clearly, such $B_{n}$ contains a $K \in \mathbb{K}$.

Our constructions of almost continuous functions will rely on the following result, that implicitly is already in $[6] .{ }^{13}$

Lemma 2.2.5. For every $K \in \mathbb{K}$, the following holds.

(i) There exists a $\hat{g} \in \mathcal{G}$ contained in $K$ and with $\operatorname{dom}(\hat{g})$ dense in $\pi[K]$.

(ii) If $\hat{g}$ is as (i) and $g \in \mathcal{G}$ is such that $\operatorname{dom}(g \cap \hat{g})$ is dense in some nontrivial interval $J$, then $g \uparrow_{J} \subseteq K$.

Proof. (i) is a well known fact. The map $h: \pi[K] \rightarrow \mathbb{R}, h(x)=\inf \{y:\langle x, y\rangle \in K\}$, is of Baire class one. ${ }^{14}$ So, the set $G$ of its points of continuity is a dense $G_{\delta}$-subset of $\pi[K]{ }^{15}$ In particular, $\hat{g}:=h \uparrow_{G}$ is as needed. ${ }^{16}$

To see (ii), first notice that

$$
g\left\lceil_ { J } \subseteq \operatorname { c l } \left(\hat{g} \cap g\left\lceil_{J}\right)\right.\right.
$$

\footnotetext{
${ }^{10}$ See e.g., [50, lemma 1].

${ }^{11}$ See e.g., [62]

${ }^{12}$ In case when $B$ is a function, $\pi[B]$ is also denoted as $\operatorname{dom}(B)$.

${ }^{13}$ Compare also [27, lemma 4.6].

${ }^{14}$ See e.g., [50, lemma 1].

${ }^{15}$ See e.g., [59, theorem 48.5].

${ }^{16}$ See also [50, lemma 1] or [24, page 117$]$.
} 
Indeed, the function $\gamma: J \cap \operatorname{dom}(g) \rightarrow g{ }_{J} \subseteq \mathbb{R}^{2}$, given as $\gamma(x):=\langle x, g(x)\rangle$, is continuous and the set $D:=\operatorname{dom}(\hat{g} \cap g)$ is dense in $J$. Therefore, we have

$$
g \uparrow_{J}=\gamma[J \cap \operatorname{dom}(g)] \subseteq \gamma[\operatorname{cl}(D)] \subseteq \operatorname{cl}(\gamma[D])=\operatorname{cl}\left(\hat{g} \cap g \uparrow_{J}\right) .
$$

Hence, $g \uparrow_{J} \subseteq \operatorname{cl}\left(g \uparrow_{J}\right) \subseteq \operatorname{cl}\left(\hat{g} \cap g \uparrow_{J}\right) \subseteq \operatorname{cl}(\hat{g}) \subseteq \operatorname{cl}(K)=K$, as needed.

The most important component in the construction of every example presented in this chapter is a result stated as the following lemma.

Lemma 2.2.6. Assume that $\operatorname{cov}_{M}=\mathfrak{c}$ holds. Let $M$ be an $F_{\sigma}$ meager subset of $\mathbb{R}$. Then there is a partial function $f$ from $\mathbb{R} \backslash M$ to $\mathbb{R}$ such that

(a) $|f \cap g|<\mathfrak{c}$ for every $g \in \mathcal{G}$, that is, $f$ is an SZ-function;

(b) $f$ is unbounded on every perfect $P \subseteq \mathbb{R}$ with $P \cap M=\emptyset$;

(c) $K \cap f \neq \emptyset$ for every $K \in \mathbb{K}$.

Proof. Let $\left\{g_{\xi}: \xi<\mathfrak{c}\right\}$ be an enumeration of $\mathcal{G}$. By induction on $\xi<\mathfrak{c}$, define the sequence of quadruples $\left\langle C_{\xi}, Z_{\xi}, D_{\xi}, f_{\xi}\right\rangle$ as follows.

(C) If $\operatorname{dom}\left(g_{\xi}\right) \backslash M$ is uncountable, then $C_{\xi}$ is a countable infinite set, enumerated as $\left\{x_{n}: n<\omega\right\}$ and contained in $\left(\operatorname{dom}\left(g_{\xi}\right) \backslash M\right) \backslash \bigcup_{\zeta<\xi}\left(C_{\zeta} \cup D_{\zeta}\right)$. Otherwise we put $C_{\xi}:=\emptyset$.

(Z) $Z_{\xi}:=\operatorname{dom}\left(g_{\xi}\right) \backslash \bigcup_{\zeta<\xi}\left(C_{\zeta} \cup D_{\zeta} \cup \operatorname{dom}\left(g_{\zeta} \cap g_{\xi}\right)\right)$.

(D) $D_{\xi}$ is a dense at most countable subset of $Z_{\xi} \backslash\left(C_{\xi} \cup M\right)$.

(F) $f_{\xi}: C_{\xi} \cup D_{\xi} \rightarrow \mathbb{R}$ is defined as:

(i) $f_{\xi}\left(x_{n}\right) \in(n, \infty) \backslash\left\{g_{\zeta}\left(x_{n}\right): \zeta<\xi\right\}$ for every $x_{n} \in C_{\xi}$;

(ii) $f_{\xi}(x)=g_{\xi}(x)$ on $D_{\xi}$.

The choice of a set $C_{\xi}$ is possible, since an uncountable $G_{\delta}$-set $\operatorname{dom}\left(g_{\xi}\right) \backslash M$ has cardinality c. It is also clear that $f:=\bigcup_{\xi<\mathfrak{c}} f_{\xi}$ is a partial function from $\mathbb{R} \backslash M$ to $\mathbb{R}$.

To see (a), notice that for every $g \in \mathcal{G}$ there exists a $\zeta<\mathfrak{c}$ such that $g=g_{\zeta}$ and that for every $\xi>\zeta$ we have $g_{\zeta} \cap f_{\xi}=\emptyset$ : on $C_{\xi}$ it is ensured by (i), while on $D_{\xi}$ by (ii) and the fact that $\operatorname{dom}\left(g_{\zeta} \cap g_{\xi}\right)$ is disjoint with $Z_{\xi} \supseteq D_{\xi}$. Hence, $f \cap g=f \cap g_{\xi}$ is a subset of $\bigcup_{\eta \leq \xi} f_{\eta}$ which has cardinality $<\mathfrak{c}$ as a union of $<\mathfrak{c}$-many countable sets. So, indeed, $|f \cap g|<\mathfrak{c}$. Hence, by a remark just before Proposition 2.1.4, $f$ is an SZ-function. 
To see (b), notice that for every perfect $P \subseteq \mathbb{R}$ with $P \cap M=\emptyset$, there exists a $\xi<\mathfrak{c}$ such that $\operatorname{dom}\left(g_{\xi}\right)=P$ as every perfect set is a $G_{\delta}$ set. Then, by $(\mathrm{C})$, the set $C_{\xi} \subseteq P \cap \operatorname{dom}(f)$ is infinite and, by (i), $f$ is unbounded on it.

Finally, we will argue for (c) using Lemma 2.2.5. So, fix a $K \in \mathbb{K}$, let $\hat{g} \in \mathcal{G}$ be contained in $K$ and with $\operatorname{dom}(\hat{g})$ dense in $\pi[K]$, and choose a $\xi<\mathfrak{c}$ with $g_{\xi}=\hat{g}$. Define

$$
\alpha:=\min \left\{\zeta \leq \xi: \operatorname{dom}\left(g_{\zeta} \cap g_{\xi}\right) \text { is somewhere dense in } \mathbb{R}\right\} .
$$

It is well-defined, since $\xi$ is in the minimized set. Let $J$ denote a nontrivial interval in which $\operatorname{dom}\left(g_{\alpha} \cap g_{\xi}\right)=\operatorname{dom}\left(g_{\alpha} \cap \hat{g}\right)$ is dense. Then, by (ii) of Lemma 2.2.5, we have $g_{\alpha}\left\lceil_{J} \subseteq K\right.$. This and (ii) imply that $f\left\lceil_{D_{\alpha} \cap J}=f_{\alpha}\left\lceil_{D_{\alpha} \cap J}=g_{\alpha}\left\lceil_{D_{\alpha} \cap J} \subseteq K\right.\right.\right.$. Also, $D_{\alpha} \cap M=\emptyset$. Thus, to finish the proof of (c) it is enough to show that $D_{\alpha} \cap J \neq \emptyset$.

To see this, first notice that

$$
\operatorname{dom}\left(g_{\beta} \cap g_{\alpha}\right) \cap J \text { is nowhere-dense for every } \beta<\alpha \text {. }
$$

Indeed, otherwise there exists a $\beta<\alpha$ such that $\operatorname{dom}\left(g_{\beta} \cap g_{\alpha}\right) \cap J$ is a $G_{\delta^{-}}$-set dense in some nontrivial interval $I \subseteq J$. In particular, $\operatorname{dom}\left(g_{\beta} \cap g_{\xi}\right)$ contains the set $\operatorname{dom}\left(g_{\beta} \cap g_{\alpha}\right) \cap \operatorname{dom}\left(g_{\alpha} \cap g_{\xi}\right)$, which is a $G_{\delta}$-set dense in $I$, contradicting the minimality of $\alpha$.

Now, by (2.2) and Proposition 2.1.1, the set $Z_{\alpha} \backslash\left(C_{\alpha} \cup M\right)$ is dense in $J$. Therefore, by (D), also $D_{\alpha}$ is dense in $J$, so that $D_{\alpha} \cap J \neq \emptyset$, as needed.

Theorem 2.2.7. If $\operatorname{cov}_{\mathrm{M}}=\mathfrak{c}$ holds, then $\mathrm{SZ} \cap \mathrm{AC} \backslash \mathrm{PR} \neq \emptyset$.

Proof. Let $\sigma \in \mathrm{SZ}$ and $f$ be an SZ-function from Lemma 2.2.6 used with $M=\emptyset$. We claim that $\bar{f}=f \cup \sigma\left\lceil_{\mathbb{R} \backslash \operatorname{dom}(f)}\right.$ is as needed, that is, in $\mathrm{SZ} \cap \mathrm{AC} \backslash \mathrm{PR}$.

Indeed, $\bar{f} \in \mathrm{SZ}$ by Propositions 2.1.3 and 2.1.4. Also, $\bar{f} \notin \mathrm{PR}$, since, by (b) of Lemma 2.2.6, $\bar{f} \supseteq f$ is unbounded on any perfect $P \subseteq \mathbb{R}$, and so, by Proposition 2.2.2, is perfect-road free. Finally, by (c) of Lemma 2.2.6, $\bar{f} \supseteq f$ intersects every $K \in \mathbb{K}$. So, by Proposition 2.2.4, $\bar{f} \in \mathrm{AC}$.

\subsection{2 $\mathrm{SZ} \cap \mathcal{D} \backslash(\mathrm{PR} \cup \mathrm{Conn})$}

Definition 2.2.8. A function $f: \mathbb{R} \rightarrow \mathbb{R}$ is connectivity, denoted as $f \in$ Conn, provided $f \uparrow_{S}$ is a connected subset of $\mathbb{R}^{2}$ whenever $S$ is a connected subset of $\mathbb{R}$.

This concept was first defined in a research problem [60] proposed by J. Nash in 1956. He inquired if an endomorphic map on a cell, that preserves connectedness of any connected subset of its domain 
to its graph, must have a fixed point or not. This problem was studied and given an affirmative answer by O. H. Hamilton [37] and J. Stallings [73]. In addition, J. Stallings proved also in [73] that $\mathrm{AC} \subseteq$ Conn. Notice, that the inclusion Conn $\subseteq \mathcal{D}$ is obvious. An example, under $\operatorname{cov}_{\mathrm{M}}=\mathfrak{c}$, of an additive function in $\mathrm{SZ} \cap \mathcal{D} \backslash$ Conn can be found in [64].

Definition 2.2.9. A function $f: \mathbb{R} \rightarrow \mathbb{R}$ is everywhere surjective, denoted as $f \in \mathrm{ES}$, provided $f[(a, b)]=\mathbb{R}$ for all $a<b$.

Equivalently, $f \in \mathrm{ES}$ if, and only if, $f^{-1}(y)$ is dense in $\mathbb{R}$ for every $y \in \mathbb{R}$. This class, under different names, has been studied by many authors. ${ }^{17}$ The name everywhere surjective comes from a 2005 paper [4] of R. Aron, V. I. Gurariy, and J. B. Seoane and the consecutive work of these authors. Clearly $\mathrm{ES} \subseteq \mathcal{D}$.

In what follows $\Delta$ will be defined as the diagonal, that is, $\Delta:=\left\{\langle x, x\rangle \in \mathbb{R}^{2}: x \in \mathbb{R}\right\}$.

Theorem 2.2.10. If $\operatorname{cov}_{\mathrm{M}}=\mathfrak{c}$, then $\mathrm{SZ} \cap \mathrm{ES} \backslash(\mathrm{PR} \cup \mathrm{Conn}) \neq \emptyset$ and $\mathrm{SZ} \cap \mathcal{D} \backslash(\mathrm{PR} \cup \mathrm{Conn}) \neq \emptyset$.

Proof. Let function $\bar{f}$ be as in the proof of Theorem 2.2.7, that is, defined as $\bar{f}=f \cup \sigma\left\lceil_{\mathbb{R} \backslash \operatorname{dom}(f)}\right.$, where $\sigma \in \mathrm{SZ}$ and $f$ is from Lemma 2.2.6 used with $M=\emptyset$. Notice that $\bar{f} \in \mathrm{ES}$, since by (c) of Lemma 2.2.6, $f$ intersects any constant map $g$ defined on any nontrivial interval. Also, by Propositions 2.1.3 and 2.1.4, $\bar{f} \in \mathrm{SZ}$.

Since $\Delta \in \mathcal{G}$, the set $D:=\operatorname{dom}(\Delta \cap \bar{f})$ has cardinality $<\mathfrak{c}$, as $\bar{f} \in$ SZ. Let $\hat{f}:=\bar{f}+\chi_{D}$, where $\chi_{D}$ is the characteristic function of the set $D$. We claim that $\hat{f} \in \mathrm{SZ} \cap \mathrm{ES} \backslash(\mathrm{PR} \cup \mathrm{Conn})$.

Indeed, $\hat{f} \notin \mathrm{PR}$ by Proposition 2.1.4, since for any perfect $P \subseteq \mathbb{R}$ the map $\hat{f}$ is unbounded. ${ }^{18}$ Also, $\hat{f} \in \mathrm{ES}$ since, for every $y \in \mathbb{R}$, the set $\bar{f}^{-1}(y)$ is dense in $\mathbb{R}$ as $\bar{f} \in \mathrm{ES}$, and so is $\hat{f}^{-1}(y)$, as it contains

$$
\bar{f}^{-1}(y) \backslash\{x \in D: \hat{f}(x)=y\} \supseteq \bar{f}^{-1}(y) \backslash\{y\}
$$

We have $\hat{f} \notin$ Conn, since $\hat{f}$ has a graph dense in $\mathbb{R}^{2}$ as $\hat{f} \in \mathrm{ES}$, and its graph does not intersect $\Delta$. Finally, $\hat{f} \in \mathrm{SZ}$ by Proposition 2.1.4, as both $\hat{f} \uparrow_{\mathbb{R} \backslash D}=\bar{f} \uparrow_{\mathbb{R} \backslash D}$ and $\hat{f} \uparrow_{D}$ are SZ.

\subsection{3 $\mathrm{SZ} \cap \mathcal{D} \cap \mathrm{CIVP} \backslash \mathrm{Conn}$}

Definition 2.2.11. A function $f: \mathbb{R} \rightarrow \mathbb{R}$ has the Cantor intermediate value property, denoted as $f \in$ CIVP, provided for every $a, b \in \mathbb{R}$ with $f(a) \neq f(b)$ and for every perfect set $K$ between $f(a)$ and $f(b)$, there exists a perfect set $C$ between $a$ and $b$ such that $f[C] \subseteq K$.

\footnotetext{
${ }^{17}$ See e.g., [36], [61], [20], or [16, section 7.2].

${ }^{18}$ This is the case, since $\hat{f}:=\bar{f}+\chi_{D}$, where $\chi_{D}$ is bounded, while $f \subseteq \bar{f}$ is unbounded on $P$.
} 
Choose a countable family $\mathcal{F}=\left\{P_{p, q} \subseteq(p, q): p<q \& p, q \in \mathbb{Q}\right\}$ of pairwise disjoint nowheredense perfect sets and define

$$
\hat{M}:=\bigcup \mathcal{F}
$$

Notice that $\hat{M}$ is a meager and an $F_{\sigma}$-subset of $\mathbb{R}$. Also, for every $P_{p, q} \in \mathcal{F}$ let

$$
\left\{C_{p, q, \xi}: \xi<\mathfrak{c}\right\}
$$

be a partition of $P_{p, q}$ consisting of perfect sets. It exists, since $P_{p, q}$ is homeomorphic to $\mathfrak{C}^{2}$, where $\mathfrak{C}$ is the Cantor set, and the sets $\{\{x\} \times \mathfrak{C}: x \in \mathfrak{C}\}$ form its partition. Thus,

$$
\left\{C_{p, q, \xi}: \xi<\mathfrak{c} \& p<q \& p, q \in \mathbb{Q}\right\}
$$

is a partition of $\hat{M}$.

Lemma 2.2.12. Let $\hat{M}$ be as in (2.3). Then there exists a function $h: \hat{M} \rightarrow \mathbb{R}$ such that

(a) $|h \cap g|<\mathfrak{c}$ for every $g \in \mathcal{G}$, that is, $h$ is an SZ-function;

(b) for every perfect $K \subseteq \mathbb{R}$ and $a<b$ there exists a perfect $P \subseteq \hat{M} \cap(a, b)$ such that $h[P] \subseteq K$.

Proof. Let $\left\{g_{\xi}: \xi<\mathfrak{c}\right\},\left\{x_{\zeta}: \zeta<\mathfrak{c}\right\}$, and $\left\{K_{\xi}: \xi<\mathfrak{c}\right\}$ be the enumerations of $\mathcal{G}, \hat{M}$, and the family of all perfect subsets of $\mathbb{R}$, respectively. Let sets $C_{p, q, \xi}$ be as in (2.4). By induction on $\zeta<\mathfrak{c}$, define a function $h: \hat{M} \rightarrow \mathbb{R}$ such that

$$
h\left(x_{\zeta}\right) \in K_{\xi} \backslash\left\{g_{\eta}\left(x_{\zeta}\right): \eta<\zeta \& x_{\zeta} \in \operatorname{dom}\left(g_{\eta}\right)\right\},
$$

where $\xi<\mathfrak{c}$ is such that $x_{\zeta} \in C_{p, q, \xi}$ for some $p, q \in \mathbb{Q}, p<q$. This completes the construction.

To see (a), notice that for every $g \in \mathcal{G}$, there exists a $\xi<\mathfrak{c}$ such that $g=g_{\xi}$ and $h\left(x_{\zeta}\right) \neq g_{\xi}\left(x_{\zeta}\right)$ for every $\zeta>\xi$. Thus, $|h \cap g| \leq \xi<\mathfrak{c}$.

To see (b), take a perfect $K \subseteq \mathbb{R}$ and $a<b$. Find $p, q \in \mathbb{Q}$ with $a<p<q<b$ and a $\xi<\mathfrak{c}$ such that $K=K_{\xi}$. Then $P:=C_{p, q, \xi} \subseteq \hat{M} \cap(a, b)$ is perfect and, according to the construction, $h[P]=h\left[C_{p, q, \xi}\right] \subseteq K_{\xi}=K$, as needed.

Theorem 2.2.13. If $\operatorname{cov}_{\mathrm{M}}=\mathfrak{c}$, then $\mathrm{SZ} \cap \mathrm{ES} \cap \mathrm{CIVP} \backslash \mathrm{Conn} \neq \emptyset$ and $\mathrm{SZ} \cap \mathcal{D} \cap \mathrm{CIVP} \backslash \operatorname{Conn} \neq \emptyset$.

Proof. Take the SZ-functions: $h: \hat{M} \rightarrow \mathbb{R}$ from Lemma 2.2.12, $f$ from Lemma 2.2.6 with $M:=\hat{M}$, and an arbitrary $\sigma \in \mathrm{SZ}$. Let

$$
\bar{f}=f \cup h \cup \sigma\lceil\mathbb{R} \backslash \operatorname{dom}(f \cup h) .
$$


Then $\bar{f} \in \mathrm{ES}$, since by (c) of Lemma 2.2.6, $f$ intersects any constant map $g$ defined on any nontrivial interval. Also, by Proposition 2.1.4, we have $\bar{f} \in \mathrm{SZ}$. So, the set $D:=\operatorname{dom}(\Delta \cap \bar{f})$ has cardinality $<\mathfrak{c}$, as $\Delta \in \mathcal{G}$. Let

$$
\hat{f}:=\bar{f}+\chi_{D}
$$

where $\chi_{D}$ is the characteristic function of $D$. We claim that $\hat{f}$ is as needed, that is, that $\hat{f} \in$ $\mathrm{SZ} \cap \mathrm{ES} \cap \mathrm{CIVP} \backslash$ Conn.

Indeed, $\hat{f} \in \mathrm{SZ}$ by Proposition 2.1.4, as a union of two SZ-functions: $\hat{f}\left\lceil_{D}\right.$ and $\hat{f}\left\lceil_{\mathbb{R} \backslash D}=\left.\bar{f}\right|_{\mathbb{R} \backslash D}\right.$. Also, $\hat{f} \in \mathrm{ES}$ since, for every $y \in \mathbb{R}$, the set $\bar{f}^{-1}(y)$ is dense in $\mathbb{R}$ as $\bar{f} \in \mathrm{ES}$, and so is $\hat{f}^{-1}(y)$, as it contains

$$
\bar{f}^{-1}(y) \backslash\{x \in D: \hat{f}(x)=y\} \supseteq \bar{f}^{-1}(y) \backslash\{y\}
$$

We have $\hat{f} \notin$ Conn, since $\hat{f}$ has a graph dense in $\mathbb{R}^{2}$ (as $\hat{f} \in \mathrm{ES}$ ) and its graph does not intersect $\Delta$.

To finish the proof, it remains to check that $\hat{f} \in \operatorname{CIVP}$. So, fix $a<b$ with $\hat{f}(a) \neq \hat{f}(b)$ and a perfect set $K$ between $\hat{f}(a)$ and $\hat{f}(b)$. We need to find a perfect $C \subseteq(a, b)$ with $\hat{f}[C] \subseteq K$. By (b) of Lemma 2.2.12, there exists a perfect $P \subseteq \hat{M} \cap(a, b)$ such that $h[P] \subseteq K$. Since $|D|<\mathfrak{c}$, there exists a perfect $C \subseteq P \backslash D$. But $\hat{f}=\bar{f}=h$ on $P \backslash D$. Hence, $\hat{f}[C]=h[C] \subseteq h[P] \subseteq K$, as needed.

\subsection{4 $\mathrm{SZ} \cap \mathcal{D} \cap \mathrm{PR} \backslash(\mathrm{Conn} \cup \mathrm{CIVP})$}

To construct a map in $\mathrm{SZ} \cap \mathrm{ES} \cap \mathrm{PR} \backslash(\mathrm{Conn} \cup \mathrm{CIVP})$, we need yet another lemma, where $\mathfrak{C}$ denotes the classic Cantor ternary set in $[0,1]$.

Lemma 2.2.14. Let $\hat{M}$ be as in (2.3). Then there exists a function $h: \hat{M} \rightarrow \mathbb{R}$ such that

(a) $|h \cap g|<\mathfrak{c}$ for every $g \in \mathcal{G}$, that is, $h$ is an SZ-function;

(b) $h[\hat{M}] \cap \mathfrak{C}=\emptyset$;

(c) for any $\langle s, t\rangle \in \mathbb{R}^{2}$ there is a perfect set $P \subseteq \hat{M} \cup\{s\}$ having s as a bilateral limit point and such that $\lim _{x \rightarrow s, x \in P} h(x)=t$.

Proof. Let $\left\{\left\langle s_{\xi}, t_{\xi}\right\rangle: \xi<\mathfrak{c}\right\}$ be an enumeration of $\mathbb{R}^{2}$ and let sets $C_{p, q, \xi}$ be as in (2.4). For every $\xi<\mathfrak{c}$, let $\left\{p_{n}^{\xi}\right\}_{n \in \mathbb{N}}$ and $\left\{q_{n}^{\xi}\right\}_{n \in \mathbb{N}}$ be the sequences of rational numbers converging to $s_{\xi}$, the first strictly increasing, the second strictly decreasing. Define

$$
P_{\xi}=\bigcup_{n \in \mathbb{N}}\left(C_{p_{n}, p_{n+1}, \xi} \cup C_{q_{n+1}, q_{n}, \xi}\right)
$$


Note that $\left\{P_{\xi}: \xi<\mathfrak{c}\right\}$ is a family of pairwise disjoint subsets of $\hat{M}$ and that, for every $\xi<\mathfrak{c}$, the set $P_{\xi} \cup\left\{s_{\xi}\right\}$ is perfect with $s_{\xi}$ being its bilateral limit point.

Let $\left\{x_{\zeta}: \zeta<\mathfrak{c}\right\}$ be an enumeration of $\hat{M}$. By induction on $\zeta<\mathfrak{c}$, define a function $h: \hat{M} \rightarrow \mathbb{R}$ so that

$$
h\left(x_{\zeta}\right) \in\left(t_{\xi}, t_{\xi}+\left|x_{\zeta}-s_{\xi}\right|\right) \backslash\left(\mathfrak{C} \cup\left\{g_{\eta}\left(x_{\zeta}\right): \eta<\zeta \& x_{\zeta} \in \operatorname{dom}\left(g_{\eta}\right)\right\}\right)
$$

when $x_{\zeta}$ is in some $P_{\xi}$ and

$$
h_{\xi}\left(x_{\zeta}\right) \in \mathbb{R} \backslash\left(\mathfrak{C} \cup\left\{g_{\eta}\left(x_{\zeta}\right): \eta<\zeta \& x_{\zeta} \in \operatorname{dom}\left(g_{\eta}\right)\right\}\right)
$$

otherwise. Such a choice is possible since $\left(y_{\xi}, y_{\xi}+\left|x_{\zeta}-x_{\xi}\right|\right) \backslash \mathfrak{C}$ has cardinality $\mathfrak{c}$, while the set $\left\{g_{\eta}\left(x_{\zeta}\right): \eta<\zeta \& x_{\zeta} \in \operatorname{dom}\left(g_{\eta}\right)\right\}$ has a smaller cardinality. This completes the construction.

Clearly, $h$ satisfies (a) and (b). To see (c), fix an $\langle s, t\rangle \in \mathbb{R}^{2}$ and let $\xi<\mathfrak{c}$ be such that $\left\langle s_{\xi}, t_{\xi}\right\rangle=$ $\langle s, t\rangle$. Then $P:=P_{\xi} \cup\left\{s_{\xi}\right\}$ is as needed, since our construction ensures that $\left|h(x)-t_{\xi}\right|<\left|x-s_{\xi}\right|$ for every $x \in P_{\xi}$.

Theorem 2.2.15. If $\operatorname{cov}_{\mathrm{M}}=\mathfrak{c}$ holds, then $\mathrm{SZ} \cap \mathrm{ES} \cap \mathrm{PR} \backslash(\mathrm{Conn} \cup \mathrm{CIVP}) \neq \emptyset$, and so also $\mathrm{SZ} \cap \mathcal{D} \cap \mathrm{PR} \backslash(\mathrm{Conn} \cup \mathrm{CIVP}) \neq \emptyset$.

Proof. Take the SZ-functions: $h: \hat{M} \rightarrow \mathbb{R}$ from Lemma 2.2.14, $f$ from Lemma 2.2.6 with $M:=\hat{M}$, and an arbitrary $\sigma \in \mathrm{SZ}$. Let

$$
\bar{f}=f \cup h \cup \sigma\left\lceil_{\mathbb{R} \backslash \operatorname{dom}(f \cup h)} .\right.
$$

Then $\bar{f} \in \mathrm{ES}$, since by (c) of Lemma 2.2.6, $f$ intersects any constant map $g$ defined on any nontrivial interval. Also, by Proposition 2.1.4, we have $\bar{f} \in \mathrm{SZ}$. So, the set $D:=\operatorname{dom}(\Delta \cap \bar{f})$ has cardinality $<\mathfrak{c}$, as $\Delta \in \mathcal{G}$. Let $\hat{\chi}: D \rightarrow \mathbb{R} \backslash \mathfrak{C}$ be one-to-one and such that $\hat{\chi} \cap \Delta=\emptyset$. Define

$$
\hat{f}:=\hat{\chi} \cup \bar{f} \uparrow_{\mathbb{R} \backslash D} .
$$

We claim that $\hat{f}$ is as needed, that is, that $\hat{f} \in \mathrm{SZ} \cap \mathrm{ES} \cap \mathrm{PR} \backslash(\mathrm{Conn} \cup \mathrm{CIVP})$.

Indeed, $\hat{f} \in \mathrm{SZ}$ by Proposition 2.1.4, as a union of two SZ-functions: $\hat{f}\left\lceil_{D}\right.$ and $\hat{f}\left\lceil_{\mathbb{R} \backslash D}=\bar{f}\left\lceil_{\mathbb{R} \backslash D}\right.\right.$. Also, $\hat{f} \in \mathrm{ES}$ since, for every $y \in \mathbb{R}$, the set $\bar{f}^{-1}(y)$ is dense in $\mathbb{R}$ as $\bar{f} \in \mathrm{ES}$, and so is $\hat{f}^{-1}(y)$, as it contains

$$
\bar{f}^{-1}(y) \backslash\{x \in D: \hat{f}(x)=y\} \supseteq \bar{f}^{-1}(y) \backslash \hat{\chi}^{-1}(y) .
$$

We have $\hat{f} \notin$ Conn, since $\hat{f}$ has a graph dense in $\mathbb{R}^{2}$ as $\hat{f} \in \mathrm{ES}$ and its graph does not intersect $\Delta$. 
For $\hat{f} \notin \mathrm{CIVP}$, notice that $\hat{f} \in \mathrm{ES}$ implies the existence of $a<b$ for which $\mathfrak{C}$ is between $\bar{f}(a)$ and $\bar{f}(b)$. Thus, it is enough to show, that $\hat{f}[C] \nsubseteq \mathfrak{C}$ for every perfect $C \subseteq \mathbb{R}$. So, by way of contradiction, assume that there is a perfect $C \subseteq \mathbb{R}$ with $\hat{f}[C] \subseteq \mathfrak{C}$. Since $|D|<\mathfrak{c}$, there is a perfect $P \subseteq C \backslash D$, for which of course $\hat{f}[P] \subseteq \mathfrak{C}$. Then, $P \cap \hat{M}=\emptyset$, since, by (b) of Lemma 2.2.14, for every $x \in \hat{M} \backslash D$ we have $\hat{f}(x)=h(x) \notin \mathfrak{C}$. So, $\hat{f}=f$ on $P$ and, by (b) of Lemma 2.2.6, $\hat{f}[P]=f[P]$ is unbounded, contradicting $\hat{f}[P] \subseteq \mathfrak{C}$.

To finish the proof, we need to show that $\hat{f} \in \mathrm{PR}$. To see this, fix an $s \in \mathbb{R}$. We need to find a perfect $P \subseteq \mathbb{R}$ having $s$ as a bilateral limit point such that $\hat{f} \uparrow_{P}$ is continuous at $s$. For this, let $t=\hat{f}(s)$. By (c) of Lemma 2.2.14, there is a perfect set $P \subseteq \hat{M} \cup\{s\}$ having $s$ as a bilateral limit point and such that $\lim _{x \rightarrow s, x \in P} h(x)=t$. Since $|D|<\mathfrak{c}$, we can decrease $P$ so that $P \backslash\{s\}$ is disjoint with $D$. But then, $\lim _{x \rightarrow s, x \in P} \hat{f}(x)=\lim _{x \rightarrow s, x \in P} h(x)=t=\hat{f}(s)$, that is, $\hat{f} \uparrow_{P}$ is continuous at $s$, as needed.

\subsection{5 $\mathrm{SZ} \cap \mathrm{Conn} \cap \mathrm{CIVP} \backslash \mathrm{AC}$}

It is well known, ${ }^{19}$ that

Proposition 2.2.16. If $f \in \mathbb{R}^{\mathbb{R}}$ intersects every compact connected subset $H$ of $\mathbb{R}^{2}$ with $|\pi[H]|>1$, then $f \in$ Conn.

In fact, this follows easily from a theorem, that if two points of the plane are separated by a closed set $F$, then they are separated by a component of $F$.

On the other hand, J. H. Roberts constructed in [69] a subset $Z \subseteq[0,1]^{2}$ homeomorphic to the Cantor set $\mathfrak{C}$ which is a zero-dimensional blocking set for functions from $[0,1]$ to $[0,1]$, that is, such that $Z \cap g \neq \emptyset$ for every continuous $g:[0,1] \rightarrow[0,1]$. This construction was modified by K. Ciesielski and A. Rosłanowski in [25, lemma 2.1] to obtain a zero-dimensional blocking set $\bar{Z}$ for functions from $\mathbb{R}$ to $\mathbb{R}$. The following proposition describes the properties of this set that we will use in what follows.

Proposition 2.2.17. Let $X:=(-1,1) \cap \mathbb{Q}$ and $G:=(-1,1) \backslash \mathbb{Q}$. There exists an embedding $F=\left\langle F_{0}, F_{1}\right\rangle: \mathbb{R} \rightarrow(-1,1) \times \mathbb{R}$ such that $F_{0}$ is non-decreasing,

(a) $B:=F[\mathbb{R}]$ is a blocking set;

(b) zero-dimensional $\bar{Z}:=F[\mathbb{Z}+\mathfrak{C}] \subseteq B$ is also a blocking set;

(c) $\gamma:=\bar{Z} \cap \pi^{-1}(G)=B \cap \pi^{-1}(G)$ is a continuous function on $G$; and

\footnotetext{
${ }^{19}$ See e.g., $[45$, theorem 2] or [33].
} 
(d) for every $x \in X$ the vertical section $B \cap \pi^{-1}(\{x\})$ of $B$ is a nontrivial closed interval and $\bar{Z} \cap \pi^{-1}(\{x\})$ consists of the two endpoints of that interval.

Using Robert's set $Z$ is relatively easy to construct a connectivity function $f:[0,1] \rightarrow[0,1]$ which

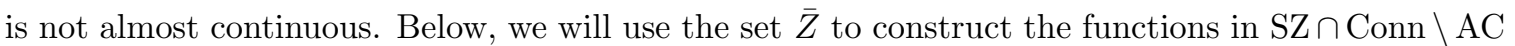
with the additional properties we examine. An example of additive function in SZ $\cap$ Conn $\backslash \mathrm{AC}$ has been constructed, under the Continuum Hypothesis, in [64, example 9]. The key result for this construction is the following lemma.

Lemma 2.2.18. Let $H$ be a compact connected subset of $\mathbb{R}^{2}$ with $|\pi[H]|>1$. If $H$ contains no vertical section of the set $B \cap \pi^{-1}(X)$ from Proposition 2.2.17, then there is a $K \in \mathbb{K}$ contained in $H \backslash \bar{Z}$.

Proof. If $\pi[H] \nsubseteq[-1,1]$, then there are $a<b$ with $[a, b] \subseteq \pi[H] \backslash[-1,1]$ and $K:=H \cap \pi^{-1}([a, b])$ is as needed. So, assume that $\pi[H] \subseteq[-1,1]$. Since $\pi[H]$ has more than one element and is connected, there is an $x \in X$ that belongs to the interior of $\pi[H]$. Let $c<d$ be such that $B \cap \pi^{-1}(\{x\})=$ $\{x\} \times[c, d]$. See Fig. 2.3. Since $H$ contains no vertical section of the set $B$, there is a $y \in(c, d)$ with $\langle x, y\rangle \notin H$. Let $s, t \in \mathbb{R}$ be such that $F(s)=\langle x, d\rangle$ and $F(t)=\langle x, c\rangle$. We assume that $s<t$, the other case being similar. ${ }^{20}$ Then, $F_{1}(s)=d>y>F_{1}(t)$ and there exist $p \in(-1, x) \cap \pi[H]$ and $q \in(x, 1) \cap \pi[H]$ such that $F_{1}(u)>y>F_{1}(v)$ for every $u \in[p, x]$ and $v \in[x, q]$. See Fig. 2.3. Also, we can assume that $[p, q] \times\{y\}$ is disjoint with $H$ (e.g., by imposing that the length of $[p, q]$ is less than the distance from $\langle x, y\rangle$ to $H)$.

Now, if $[p, x) \subseteq \pi[H \cap([p, x) \times(-\infty, y])]$, then the set $K:=H \cap([p, m] \times[y, \infty))$, for any $m \in(p, x)$, is as needed. See the left part of Fig. 2.3. Similarly, if $(x, q] \subseteq \pi[H \cap((x, q] \times[y, \infty))]$, then the set $K:=H \cap\left(\left[m^{\prime}, q\right] \times[y, \infty)\right)$, for any $m^{\prime} \in(x, q)$, satisfies the lemma. Therefore, by way of contradiction, assume that neither of this happens. Then, there are $u \in[p, x)$ and $v \in(x, q]$ such that the set

$$
L:=(\{u\} \times(-\infty, y]) \cup([u, v] \times\{y\}) \cup(\{v\} \times[y, \infty))
$$

is disjoint with $H$, see the right part of Fig. 2.3. But this is impossible, since such $L$ separates $H \cap(\{p\} \times \mathbb{R}) \neq \emptyset$ from $H \cap(\{q\} \times \mathbb{R}) \neq \emptyset$, contradicting the connectedness of $H$.

Theorem 2.2.19. If $\operatorname{cov}_{\mathrm{M}}=\mathfrak{c}$ holds, then $\mathrm{SZ} \cap \operatorname{Conn} \cap \mathrm{CIVP} \backslash \mathrm{AC} \neq \emptyset$.

Proof. Similarly as in the proof of Theorem 2.2.13, take the following SZ-functions: $h: \hat{M} \rightarrow \mathbb{R}$ from Lemma 2.2.12, $f$ from Lemma 2.2.6, this time with $M:=\hat{M} \cup X$ where $X$ is from Proposition 2.2.17,

\footnotetext{
${ }^{20}$ Actually, the other case cannot happen in the actual construction of the curve from Proposition 2.2.17.
} 

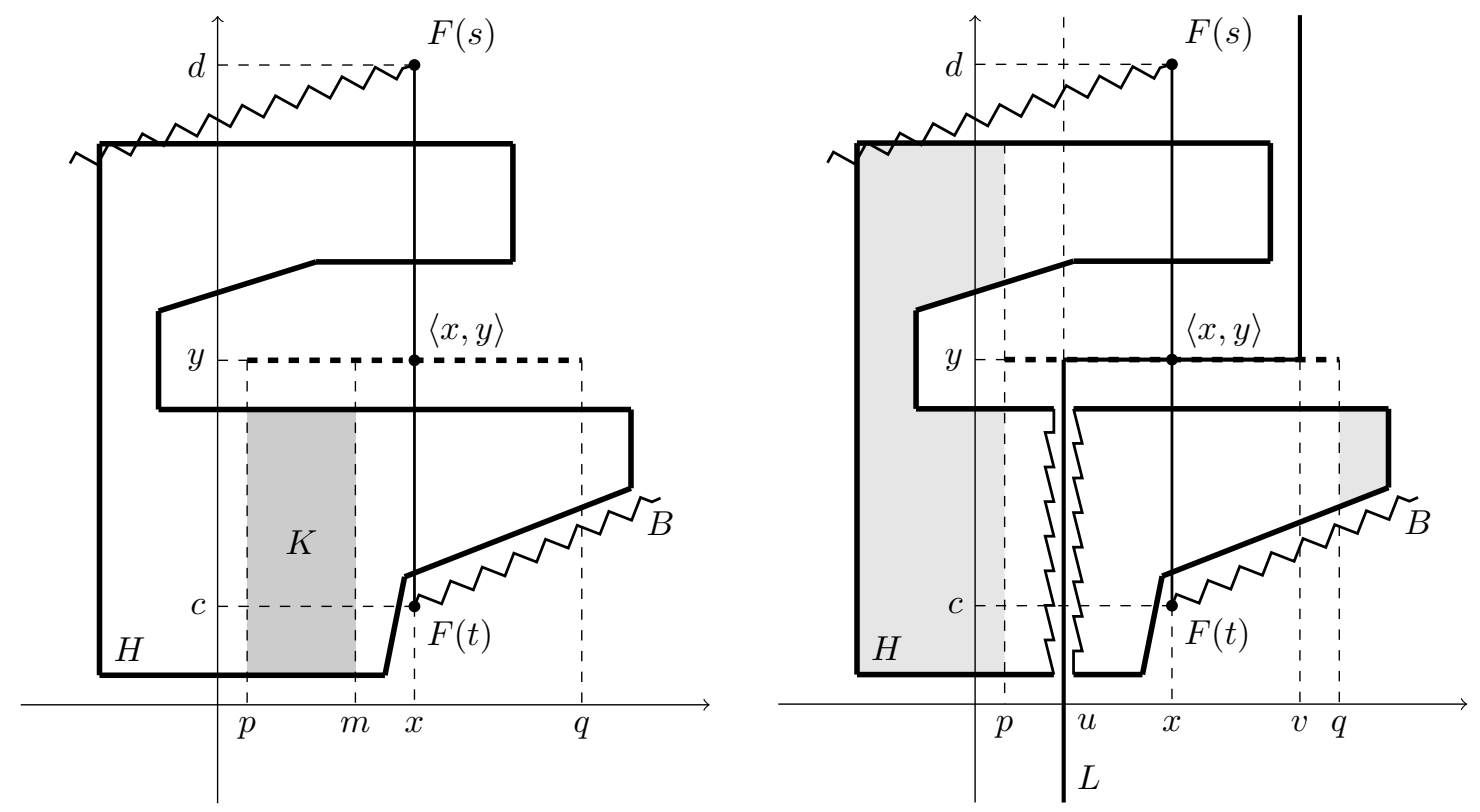

Figure 2.3: Illustration for the proof of Lemma 2.2.18. Left figure corresponds to the case when $[p, x) \subseteq \pi[H \cap([p, x) \times(-\infty, y])]$. The right figure addresses the case leading to the contradiction

and an arbitrary $\sigma \in \mathrm{SZ}$. Let

$$
\bar{f}=f \cup h \cup \sigma\left\lceil_{\mathbb{R} \backslash \operatorname{dom}(f \cup h)} .\right.
$$

Then, by Proposition 2.1.4, we have $\bar{f} \in \mathrm{SZ}$. So, the set $D:=X \cup \operatorname{dom}(\gamma \cap \bar{f})$ has cardinality $<\mathfrak{c}$, where $\gamma \in \mathcal{G}$ is as in (c) of Proposition 2.2.17. Moreover, $\operatorname{dom}(\bar{Z} \cap \bar{f}) \subseteq D$. Let $\hat{\chi}: D \rightarrow \mathbb{R}$ be such that $\hat{\chi} \cap \bar{Z}=\emptyset$ and $\left.\hat{\chi}\right|_{X} \subseteq B \backslash \bar{Z}$, where $\bar{Z}$ and $B$ are from Proposition 2.2.17. Define

$$
\hat{f}:=\hat{\chi} \cup \bar{f} \uparrow_{\mathbb{R} \backslash D} .
$$

We claim that $\hat{f}$ is as needed, that is, that $\hat{f} \in \mathrm{SZ} \cap \operatorname{Conn} \cap \operatorname{CIVP} \backslash \mathrm{AC}$.

Indeed, $\hat{f} \in \mathrm{SZ}$ by Proposition 2.1.4, as a union of two SZ-functions: $\hat{f} \uparrow_{D}$ and $\hat{f}\left\lceil_{\mathbb{R} \backslash D}=\bar{f}\left\lceil_{\mathbb{R} \backslash D}\right.\right.$. Clearly, $\hat{f} \notin \mathrm{AC}$, since $\hat{f} \cap \bar{Z}=\emptyset$, while $\bar{Z}$ is a blocking set.

An argument that $\hat{f} \in$ CIVP is identical to one presented in Theorem 2.2.13.

Finally, to see that $\hat{f} \in$ Conn, fix a compact connected subset $H$ of $\mathbb{R}^{2}$ with $|\pi[H]|>1$. By Proposition 2.2.16 it is enough to show that $\hat{f} \cap H \neq \emptyset$. This is clear when $H$ contains a vertical section of the set $\bar{Z} \cap \pi^{-1}(X)$, since we ensured that $\hat{\chi} \subseteq \hat{f}$ intersects every such $H$. But otherwise, by Lemma 2.2.18, there is a $K \in \mathbb{K}$ contained in $H \backslash \bar{Z}$. Also, by Lemma 2.2.6, there an $x \in \operatorname{dom}(f)$ such that $\langle x, f(x)\rangle \in K \subseteq H$. So, to finish the proof, it is enough to notice that $\hat{f}(x)=f(x)$. Indeed, this is the case, since $x \notin D: x \notin X$, as $\operatorname{dom}(f)$ is disjoint from $M=\hat{M} \cup X$; and $x \notin \operatorname{dom}(\gamma \cap \bar{f})$ 
since $K$ is disjoint from $\bar{Z} \supseteq \gamma$.

\subsection{6 $\mathrm{SZ} \cap \mathrm{Conn} \cap \mathrm{PR} \backslash(\mathrm{AC} \cup \mathrm{CIVP})$}

The proof of the next theorem is a simple mix of the elements of the proofs of Theorems 2.2.15 and 2.2.19.

Theorem 2.2.20. If $\operatorname{cov}_{\mathrm{M}}=\mathfrak{c}$ holds, then $\mathrm{SZ} \cap \mathrm{Conn} \cap \mathrm{PR} \backslash(\mathrm{AC} \cup \mathrm{CIVP}) \neq \emptyset$.

Proof. Similarly as in the proof of Theorem 2.2.15, take the following SZ-functions: $h: \hat{M} \rightarrow \mathbb{R}$ from Lemma 2.2.14, $f$ from Lemma 2.2.6 with $M:=\hat{M} \cup X$ where set $X$ is from Proposition 2.2.17, and $\sigma: \mathbb{R} \rightarrow \mathbb{R}$. Let

$$
\bar{f}=f \cup h \cup \sigma\left\lceil_{\mathbb{R} \backslash \operatorname{dom}(f \cup h)} .\right.
$$

Then $\bar{f} \in \mathrm{ES}$, since by (c) of Lemma 2.2.6, $f$ intersects any constant map $g$ defined on any nontrivial interval. Also, by Proposition 2.1.4, we have $\bar{f} \in \mathrm{SZ}$. So, the set $D:=X \cup \operatorname{dom}(\gamma \cap \bar{f})$ has cardinality $<\mathfrak{c}$, where $\gamma \in \mathcal{G}$ is as in (c) of Proposition 2.2.17. Moreover, $\operatorname{dom}(\bar{Z} \cap \bar{f}) \subseteq D$. Let $\hat{\chi}: D \rightarrow \mathbb{R}$ such that $\hat{\chi} \cap \bar{Z}=\emptyset$ and $\hat{\chi} \mid X \subseteq B \backslash \bar{Z}$, where $\bar{Z}$ and $B$ are from Proposition 2.2.17. Define

$$
\hat{f}:=\hat{\chi} \cup \bar{f}\left\lceil_{\mathbb{R} \backslash D} .\right.
$$

We claim that $\hat{f}$ is as needed, that is, that $\hat{f} \in \mathrm{SZ} \cap \mathrm{Conn} \cap \mathrm{PR} \backslash(\mathrm{AC} \cup \mathrm{CIVP})$.

Indeed, $\hat{f} \in \mathrm{SZ}$ by Proposition 2.1.4, as being a union of two SZ-functions: $\hat{\chi}$ and $\bar{f} \uparrow_{\mathbb{R} \backslash D}$. Clearly, $\hat{f} \notin \mathrm{AC}$, since $\hat{f} \cap \bar{Z}=\emptyset$, while $\bar{Z}$ is a blocking set.

The arguments for $\hat{f} \notin \mathrm{CIVP}$ and $\hat{f} \in \mathrm{PR}$ are the same with those used in Theorem 2.2.15, while $\hat{f} \in$ Conn can be argued as in Theorem 2.2.19.

\subsection{7 $\mathrm{SZ} \cap \mathrm{Conn} \backslash(\mathrm{AC} \cup \mathrm{PR})$}

Similarly, the proof of this last theorem in this section is a mix of the elements of proofs of Theorems 2.2.10 and 2.2.19.

Theorem 2.2.21. If $\operatorname{cov}_{\mathrm{M}}=\mathfrak{c}$ holds, then $\mathrm{SZ} \cap \operatorname{Conn} \backslash(\mathrm{AC} \cup \mathrm{PR}) \neq \emptyset$.

Proof. Take the SZ-functions: $f$ from Lemma 2.2.6 with $M:=X$ from Proposition 2.2.17, $\sigma \in \mathrm{SZ}$, and $\hat{X}: X \rightarrow \mathbb{R}$ such that $\hat{X} \subseteq B \backslash \bar{Z}$. Let

$$
\bar{f}=f \cup \hat{X} \cup \sigma \uparrow_{\mathbb{R} \backslash \operatorname{dom}(f \cup \hat{X})} .
$$


Then, by Proposition 2.1.4, we have $\bar{f} \in \mathrm{SZ}$. So, the set $D:=\operatorname{dom}(\gamma \cap \bar{f})$ has cardinality $<\mathfrak{c}$, where $\gamma \in \mathcal{G}$ is as in (c) of Proposition 2.2.17. Moreover, we have $\operatorname{dom}(\bar{Z} \cap \bar{f}) \subseteq D$. Define

$$
\hat{f}:=\bar{f}+\chi_{D},
$$

where $\chi_{D}$ is the characteristic function of $D$. We claim that $\hat{f}$ is as needed, that is, that $\hat{f} \in$ $\mathrm{SZ} \cap \mathrm{Conn} \cap \backslash(\mathrm{AC} \cup \mathrm{PR})$.

Indeed, $\hat{f} \in \mathrm{SZ}$ by Proposition 2.1.4 as both $\hat{f} \uparrow_{\mathbb{R} \backslash D}=\bar{f} \uparrow_{\mathbb{R} \backslash D}$ and $\hat{f} \uparrow_{D}$ are SZ. Clearly, $\hat{f} \notin \mathrm{AC}$, since $\hat{f} \cap \bar{Z}=\emptyset$, while $\bar{Z}$ is a blocking set.

To show $\hat{f} \notin \mathrm{PR}$, fix any perfect $P \subseteq \mathbb{R}$. By Proposition 2.2.2, it is enough to show that $\hat{f}$ is unbounded on $P$. To see this, choose a perfect $C \subseteq P \backslash X$. Then, by (b) of Lemma 2.2.6, $\bar{f} \supseteq f$ is unbounded on $C \subseteq P$. Therefore $\hat{f}$ is unbounded on $P$ as being the sum of an unbounded function and the other bounded.

The argument for $\hat{f} \in$ Conn is identical to one used for it in Theorem 2.2.19.

\subsection{8 $\mathrm{SZ} \cap \mathrm{AC} \cap \mathrm{PR} \backslash \mathrm{CIVP}$ and $\mathrm{SZ} \cap \mathrm{AC} \cap \mathrm{CIVP}$}

The examples of maps in these classes, being additionally additive, have been constructed under

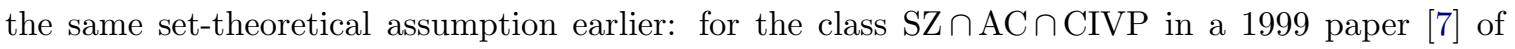
K. Banaszewski and T. Natkaniec while for the class $\mathrm{SZ} \cap \mathrm{AC} \cap \mathrm{PR} \backslash \mathrm{CIVP}$ in a 2004 paper [63] of T. Natkaniec and H. Rosen. Nevertheless, we have crafted a powerful Lemma 2.2.6 as the core of all theorems above. In particular, the lemma produces a partial function $f$ such that any extension of $f$ is almost continuous, and $\operatorname{dom}(f)$ avoids an arbitrary $F_{\sigma}$ meager subset of $\mathbb{R}$. Along with Lemmas 2.2.12 and 2.2.14, the same machinery allows us to easily build the maps in SZ $\cap \mathrm{AC} \cap \mathrm{CIVP}$ and $\mathrm{SZ} \cap \mathrm{AC} \cap \mathrm{PR} \backslash \mathrm{CIVP}$.

Theorem 2.2.22. If $\operatorname{cov}_{\mathrm{M}}=\mathfrak{c}$ holds, then $\mathrm{SZ} \cap \mathrm{AC} \cap \mathrm{CIVP} \neq \emptyset$.

Proof. The function $\bar{f}$ used in the proof of Theorem 2.2.13 is as needed. That is, if

$$
\bar{f}:=f \cup h \cup \sigma\left\lceil_{\mathbb{R} \backslash \operatorname{dom}(f \cup h)},\right.
$$

where $h: \hat{M} \rightarrow \mathbb{R}$ is from Lemma 2.2.12, $f$ is from Lemma 2.2.6 with $M:=\hat{M}$ and $\sigma \in \mathrm{SZ}$, then $\bar{f} \in \mathrm{SZ} \cap \mathrm{AC} \cap \mathrm{CIVP}$.

Indeed, $\bar{f} \in \mathrm{AC}$ by (c) of Lemma 2.2.6. Also, by Proposition 2.1.4, we have $\bar{f} \in \mathrm{SZ}$. 
To see that $\bar{f} \in \mathrm{CIVP}$, fix $a<b$ with $\bar{f}(a) \neq \bar{f}(b)$ and a perfect set $K$ between $\bar{f}(a)$ and $\bar{f}(b)$. We need to find a perfect $P \subseteq(a, b)$ with $\bar{f}[P] \subseteq K$. But, by (b) of Lemma 2.2.12, there exists a perfect $P \subseteq \hat{M} \cap(a, b)$ such that $h[P] \subseteq K$. Since $\bar{f}=h$ on $P, \bar{f}[P]=h[P] \subseteq K$, as needed.

Theorem 2.2.23. If $\operatorname{cov}_{\mathrm{M}}=\mathfrak{c}$ holds, then $\mathrm{SZ} \cap \mathrm{AC} \cap \mathrm{PR} \backslash \mathrm{CIVP} \neq \emptyset$.

Proof. The function $\bar{f}$ used in the proof of Theorem 2.2.15 is as needed. That is, if

$$
\bar{f}:=f \cup h \cup \sigma\left\lceil_{\mathbb{R} \backslash \operatorname{dom}(f \cup h)},\right.
$$

where $h: \hat{M} \rightarrow \mathbb{R}$ is from Lemma 2.2.14, $f$ is from Lemma 2.2.6 with $M:=\hat{M}$ and $\sigma \in \mathrm{SZ}$, then $\bar{f} \in \mathrm{SZ} \cap \mathrm{AC} \cap \mathrm{PR} \backslash \mathrm{CIVP}$.

Indeed, $\bar{f} \in \mathrm{AC} \cap \mathrm{ES}$ by (c) of Lemma 2.2.6. Also, by Proposition 2.1.4, we have $\bar{f} \in \mathrm{SZ}$.

For $\bar{f} \notin \mathrm{CIVP}$, notice that $\bar{f} \in \mathrm{ES}$ implies the existence of $a<b$ for which $\mathfrak{C}$ is between $\bar{f}(a)$ and $\bar{f}(b)$. Thus, it is enough to show that $\bar{f}[P] \not \subset \mathfrak{C}$ for every perfect $P \subseteq \mathbb{R}$. So, by way of contradiction, assume that there is a perfect $P \subseteq \mathbb{R}$ with $\bar{f}[P] \subseteq \mathfrak{C}$. Then, $P \cap \hat{M}=\emptyset$ since, by (b) of Lemma 2.2.14, we have $\bar{f}(x)=h(x) \notin \mathfrak{C}$ for every $x \in \hat{M}$. So, $\bar{f}=f$ on $P$ and, by (b) of Lemma $2.2 .6, \bar{f}[P]=f[P]$ is unbounded, contradicting $\hat{f}[P] \subseteq \mathfrak{C}$.

To see $\bar{f} \in \mathrm{PR}$, fix an $s \in \mathbb{R}$ and let $t=\bar{f}(s)$. By (c) of Lemma 2.2.14, there is a perfect set $P \subseteq \hat{M} \cup\{s\}$ having $s$ as a bilateral limit point and such that $\lim _{x \rightarrow s, x \in P} h(x)=t$. Since $\lim _{x \rightarrow s, x \in P} \bar{f}(x)=\lim _{x \rightarrow s, x \in P} h(x)=t=\bar{f}(s)$, we conclude that $\bar{f} \uparrow_{P}$ is continuous at $s$. 


\section{Bibliography}

[1] Gbrel M. Albkwre, Krzysztof Chris Ciesielski, and Jerzy Wojciechowski, Lineability of the functions that are Sierpiński-Zygmund, Darboux, but not connectivity, Rev. R. Acad. Cienc. Exactas Fís. Nat. Ser. A Mat. RACSAM 114 (2020), no. 3, Paper No. 145, 10, DOI 10.1007/s13398-020-00881-9. MR4109251

[2] Pieter C. Allaart and Kiko Kawamura, The Takagi function: a survey, Real Anal. Exchange 37 (2011/12), no. 1, 1-54, DOI 10.14321/realanalexch.37.1.0001. MR3016850

[3] Tom M. Apostol, Mathematical analysis, 2nd ed., Addison-Wesley Publishing Co., Reading, Mass.-London-Don Mills, Ont., 1974. MR0344384

[4] Richard Aron, V. I. Gurariy, and J. B. Seoane, Lineability and spaceability of sets of functions on $\mathbb{R}$, Proc. Amer. Math. Soc. 133 (2005), no. 3, 795-803, DOI 10.1090/S0002-9939-04-07533-1. MR2113929

[5] V. Aversa, M. Laczkovich, and D. Preiss, Extension of differentiable functions, Comment. Math. Univ. Carolin. 26 (1985), no. 3, 597-609. MR817830

[6] Marek Balcerzak, Krzysztof Ciesielski, and Tomasz Natkaniec, Sierpiński-Zygmund functions that are Darboux, almost continuous, or have a perfect road, Arch. Math. Logic 37 (1997), no. 1, 29-35, DOI 10.1007/s001530050080. MR1485861

[7] Krzysztof Banaszewski and Tomasz Natkaniec, Sierpinsski-Zygmund functions that have the Cantor intermediate value property, Real Anal. Exchange 24 (1998/99), no. 2, 827-835, DOI 10.2307/44153001. MR1704755

[8] J. Blažek, E. Borák, and J. Malý, On Köpcke and Pompeiu functions, Časopis Pěst. Mat. 103 (1978), no. 1, 53-61, 97, DOI 10.21136/CPM.1978.117970 (English, with Czech and loose Russian summaries). MR0486352

[9] Jan P. Boroński, Jiří Kupka, and Piotr Oprocha, Edrei’s conjecture revisited, Ann. Henri Poincaré 19 (2018), no. 1, 267-281, DOI 10.1007/s00023-017-0623-9. MR3743761

[10] Andrew Bruckner, Some New Simple Proofs of Old Difficult Theorems, Real Anal. Exchange 9 (1983/84), no. 1, 63-78, DOI 10.2307/44153514.

[11] _ _ Differentiation of real functions, 2nd ed., CRM Monograph Series, vol. 5, American Mathematical Society, Providence, RI, 1994. MR1274044

[12] Andrew M. Bruckner, Judith B. Bruckner, and Brian S. Thomson, Real Analysis, 2nd ed., CreateSpace Independent Publishing Platform, 1997. https://ClassicalRealAnalysis.com.

[13] Jack Ceder, Some examples on continuous restrictions, Real Anal. Exchange 7 (1981/82), no. 1, 155-162, DOI 10.2307/44153400. MR646647 
[14] C. Cellérier, Note sur les principes fondamentaux de l'analyse, Bulletin des sciences mathématiques 14 (1890), no. 2, 142-160 (French). https://books.google.com/books?id=HMghAQAAIAAJ\&lpg=PA142\&ots=FTe0fVlywQ\&pg= PA142.

[15] Monika Ciesielska and Krzysztof Chris Ciesielski, Differentiable extension theorem: a lost proof of V. Jarnik, J. Math. Anal. Appl. 454 (2017), no. 2, 883-890, DOI 10.1016/j.jmaa.2017.05.032. MR3658803

[16] Krzysztof Ciesielski, Set theory for the working mathematician, London Mathematical Society Student Texts, vol. 39, Cambridge University Press, Cambridge, 1997. MR1475462

[17] Krzysztof Chris Ciesielski, Monsters in calculus, Amer. Math. Monthly 125 (2018), no. 8, 739-744, DOI 10.1080/00029890.2018.1502011. MR3859646

[18] Krzysztof Chris Ciesielski and Jakub Jasinski, An auto-homeomorphism of a Cantor set with derivative zero everywhere, J. Math. Anal. Appl. 434 (2016), no. 2, 1267-1280, DOI 10.1016/j.jmaa.2015.09.076. MR3415721

[19] Krzysztof Ciesielski and Jan Jastrzȩbski, Darboux-like functions within the classes of Baire one, Baire two, and additive functions, Topology Appl. 103 (2000), no. 2, 203-219, DOI 10.1016/S0166-8641(98)00169-2. MR1758794

[20] Krzysztof Ciesielski and Arnold W. Miller, Cardinal invariants concerning functions whose sum is almost continuous, Real Anal. Exchange 20 (1994/95), no. 2, 657-672, DOI 10.2307/44152549. MR1348089

[21] Krzysztof C. Ciesielski and Cheng-Han Pan, Doubly paradoxical functions of one variable, J. Math. Anal. Appl. 464 (2018), no. 1, 274-279, DOI 10.1016/j.jmaa.2018.04.012. MR3794088

$[22] \_$_ Typical bad differentiable extensions, J. Math. Anal. Appl. 474 (2019), no. 1, 518-523, DOI 10.1016/j.jmaa.2019.01.061. MR3912915

[23] Krzysztof Chris Ciesielski and Cheng-Han Pan, Examples of Sierpinski-Zygmund maps in the class of Darbouxlike functions, Banach J. Math. Anal. 14 (2020), no. 2, 433-449, DOI 10.1007/s43037-019-00001-9. MR4091464

[24] Krzysztof Ciesielski and Janusz Pawlikowski, The covering property axiom, CPA, Cambridge Tracts in Mathematics, vol. 164, Cambridge University Press, Cambridge, 2004. A combinatorial core of the iterated perfect set model. MR2176267

[25] Krzysztof Ciesielski and Andrzej Rosłanowski, Two examples concerning almost continuous functions, Topology Appl. 103 (2000), no. 2, 187-202, DOI 10.1016/S0166-8641(98)00168-0. MR1758793

[26] Krzysztof C. Ciesielski and Juan B. Seoane-Sepúlveda, Differentiability versus continuity: restriction and extension theorems and monstrous examples, Bull. Amer. Math. Soc. (N.S.) 56 (2019), no. 2, 211-260, DOI 10.1090/bull/1635. MR3923344

[27] K. C. Ciesielski and J. B. Seoane-Sepúlveda, A century of Sierpiński-Zygmund functions, Rev. R. Acad. Cienc. Exactas Fís. Nat. Ser. A Mat. RACSAM 113 (2019), no. 4, 3863-3901, DOI 10.1007/s13398-019-00726-0. MR3999051

[28] Gaston Darboux, Mémoire sur les fonctions discontinues, Ann. Sci. École Norm. Sup. (2) 4 (1875), 57-112, DOI 10.24033/asens.122 (French). MR1508624

[29] Udayan B. Darji, A Sierpiński-Zygmund function which has a perfect road at each point, Colloq. Math. 64 (1993), no. 2, 159-162, DOI 10.4064/cm-64-2-159-162. MR1218479

[30] A. Denjoy, Sur les fonctions dérivées sommables, Bull. Soc. Math. France 43 (1915), 161-248, DOI 10.24033/bsmf.958 (French). MR1504743 
[31] Gerald A. Edgar (ed.), Classics on fractals, Studies in Nonlinearity, Westview Press. Advanced Book Program, Boulder, CO, 2004. MR2049443

[32] James Foran, Fundamentals of real analysis, Monographs and Textbooks in Pure and Applied Mathematics, vol. 144, Marcel Dekker, Inc., New York, 1991. MR1201817

[33] B. D. Garrett, D. Nelms, and K. R. Kellum, Characterizations of connected real functions. 1, Jber. Deutsch. Math.-Verein. 73 (1971/72), no. part, 131-137. MR486349

[34] Bernard R. Gelbaum and John M. H. Olmsted, Counterexamples in analysis, Dover Publications, Inc., Mineola, NY, 2003. Corrected reprint of the second (1965) edition. MR1996162

[35] Richard G. Gibson and Tomasz Natkaniec, Darboux like functions, Real Anal. Exchange 22 (1996/97), no. 2, 492-533, DOI 10.2307/44153937. MR1460971

[36] Israel Halperin, Discontinuous functions with the Darboux property, Amer. Math. Monthly 57 (1950), 539-540, DOI $10.2307 / 2307938$. MR38415

[37] O. H. Hamilton, Fixed points for certain noncontinuous transformations, Proc. Amer. Math. Soc. 8 (1957), 750-756, DOI 10.2307/2033293. MR87095

[38] G. A. Heuer, The derivative of the total variation function, Amer. Math. Monthly 78 (1971), 1110-1112, DOI 10.2307/2316319. MR297942

[39] John K. Hunter and Bruno Nachtergaele, Applied analysis, World Scientific Publishing Co., Inc., River Edge, NJ, 2001. MR1829589

[40] Marek Jarnicki and Peter Pflug, Continuous nowhere differentiable functions, Springer Monographs in Mathematics, Springer, Cham, 2015. The monsters of analysis. MR3444902

[41] Vojtěch Jarník, O funkci Bolzanovĕ, C̆asopis pro pěstování matematiky a fysiky 51 (1922), no. 4, 248-264, DOI 10.21136/CPMF.1922.109021 (Czech).

[42] __ O rozšǐrení definičního oboru funkcí jedné proměnné, přičemž zůstává zachována derivabilita funkce, Rozpravy Čes. akademie, II. tř. XXXII (1923), no. 15, 1-15 (Czech). https://hdl.handle.net/10338.dmlcz/ 500494.

[43] _ Sur l'extension du domaine de définition des fonctions d'une variable, qui laisse intacte la dérivabilité de la fonction, Bull. Internat. de l'Académie des Sciences de Bohême (1923), 1-5 (French). https://hdl.handle. net/10338.dmlcz/500753.

[44] M. Jašek, Funkce Bolzanova, C̆asopis pro pĕstování matematiky a fysiky 51 (1922), no. 2, 69-76, DOI 10.21136/CPMF.1922.121916 (Czech).

[45] F. B. Jones, Connected and disconnected plane sets and the functional equation $f(x)+f(y)=f(x+y)$, Bull. Amer. Math. Soc. 48 (1942), 115-120, DOI 10.1090/S0002-9904-1942-07615-4. MR5906

[46] Camille Jordan, Sur la série de Fourier, Comptes Rendus de l'Académie des Sciences Paris 2 (1881), 228-230, DOI 10.2307/2316319 (French).

[47] H. Turgay Kaptanoğlu, In praise of $y=x^{\alpha} \sin \left(\frac{1}{x}\right)$, Amer. Math. Monthly 108 (2001), no. 2, 144-150, DOI 10.2307/2695527. MR1818187

[48] Y. Katznelson and Karl Stromberg, Everywhere differentiable, nowhere monotone, functions, Amer. Math. Monthly 81 (1974), 349-354, DOI 10.2307/2318996. MR335701 
[49] Alexander S. Kechris, Classical descriptive set theory, Graduate Texts in Mathematics, vol. 156, Springer-Verlag, New York, 1995. MR1321597

[50] Kenneth R. Kellum, Almost continuity and connectivity - sometimes it's as easy to prove a stronger result, Real Anal. Exchange 8 (1982/83), no. 1, 244-252, DOI 10.2307/44151590. MR694512

[51] Martin Koc and Luděk Zajíček, A joint generalization of Whitney's $C^{1}$ extension theorem and Aversa-LaczkovichPreiss' extension theorem, J. Math. Anal. Appl. 388 (2012), no. 2, 1027-1037, DOI 10.1016/j.jmaa.2011.10.049. MR2869805

[52] Alfred Köpcke, Ueber Differentiirbarkeit und Anschaulichkeit der stetigen Functionen, Math. Ann. 29 (1887), no. 1, 123-140, DOI 10.1007/BF01445174 (German). MR1510403

[53] _ Ueber eine durchaus differentiirbare, stetige Function mit Oscillationen in jedem Intervalle, Math. Ann. 34 (1889), no. 2, 161-171, DOI 10.1007/BF01453433 (German). MR1510572

[54] _ Ueber eine durchaus differentiirbare, stetige Function mit Oscillationen in jedem Intervalle, Math. Ann. 35 (1889), no. 1-2, 104-109, DOI 10.1007/BF01443873 (German). MR1510599

[55] John M. Lee, Introduction to smooth manifolds, 2nd ed., Graduate Texts in Mathematics, vol. 218, Springer, New York, 2013. MR2954043

[56] Solomon Marcus, Sur les dérivées dont les zéros forment un ensemble frontière partout dense, Rend. Circ. Mat. Palermo (2) 12 (1963), 5-40, DOI 10.1007/BF02855906 (French). MR167572

[57] J. Mařík, Derivatives and closed sets, Acta Math. Hungar. 43 (1984), no. 1-2, 25-29, DOI 10.1007/BF01951320. MR731958

[58] I. Maximoff, Sur les fonctions ayant la propriété de Darboux, Prace Mat. Fiz. 43 (1936), no. 1, 241-265 (French). https://eudml.org/doc/215564.

[59] James R. Munkres, Topology, 2nd ed., Prentice Hall, Inc., Upper Saddle River, NJ, 2000. MR3728284

[60] J. Nash, Generalized Brouwer Theorem, Bull. Amer. Math. Soc. 62 (1956), no. 1, 76, DOI 10.1090/S0002-99041956-09993-8.

[61] Tomasz Natkaniec, On compositions and products of almost continuous functions, Fund. Math. 139 (1991), no. 1, 59-74, DOI 10.4064/fm-139-1-59-74. MR1141294

[62] T. Natkaniec, Almost continuity, Real Anal. Exchange 17 (1991/92), no. 2, 462-520, DOI 10.2307/44153745. MR1171393

[63] Tomasz Natkaniec and Harvey Rosen, An example of an additive almost continuous Sierpiński-Zygmund function, Real Anal. Exchange 30 (2004/05), no. 1, 261-265, DOI 10.14321/realanalexch.30.1.0261. MR2127530

[64] __ Additive Sierpiński-Zygmund functions, Real Anal. Exchange 31 (2005/06), no. 1, 253-269, DOI 10.14321/realanalexch.31.1.0253. MR2218841

[65] Cheng-Han Pan, Nowhere-monotone differentiable functions and bounded variation, J. Math. Anal. Appl. 494 (2021), no. 2, 124618, DOI 10.1016/j.jmaa.2020.124618. MR4153868

[66] G. Petruska and M. Laczkovich, Baire 1 functions, approximately continuous functions and derivatives, Acta Math. Acad. Sci. Hungar. 25 (1974), 189-212, DOI 10.1007/BF01901760. MR379766

[67] D. Pompeiu, Sur la continuité des fonctions de variables complexes, Ann. Fac. Sci. Toulouse Sci. Math. Sci. Phys. (2) 7 (1905), no. 3, 265-315, DOI 10.5802/afst.226 (French). MR1508277 
[68] _ Sur les fonctions dérivées, Math. Ann. 63 (1907), no. 3, 326-332, DOI 10.1007/BF01449201 (French). MR1511410

[69] J. H. Roberts, Zero-dimensional sets blocking connectivity functions, Fund. Math. 57 (1965), 173-179, DOI 10.4064/fm-57-2-173-179. MR195065

[70] H. L. Royden and P. M. Fitzpatrick, Real analysis, 4th ed., Pearson, 2010.

[71] Walter Rudin, Principles of mathematical analysis, 3rd ed., McGraw-Hill Book Co., New York-AucklandDüsseldorf, 1976. International Series in Pure and Applied Mathematics. MR0385023

[72] W. Sierpiński and A. Zygmund, Sur une fonction qui est discontinue sur tout ensemble de puissance du continu, Fund. Math. 4 (1923), 316-318, DOI 10.4064/fm-4-1-316-318.

[73] J. Stallings, Fixed point theorems for connectivity maps, Fund. Math. 47 (1959), 249-263, DOI 10.4064/fm-473-249-263. MR117710

[74] T. Takagi, A simple example of the continuous function without derivative, Tokyo Sugaku-Butsurigakkwai Hokoku 1 (1901), F176-F177, DOI 10.11429/subutsuhokoku1901.1.F176.

[75] Johan Thim, Continuous Nowhere Differentiable Functions, Master's Thesis, Luleå University of Technology, 2003.

[76] Brian S. Thomson, Judith B. Bruckner, and Andrew M. Bruckner, Elementary Real Analysis, 2nd ed., CreateSpace Independent Publishing Platform, 2008. https://ClassicalRealAnalysis.com.

[77] B. L. van der Waerden, Ein einfaches Beispiel einer nicht-differenzierbaren stetigen Funktion, Math. Z. 32 (1930), no. 1, 474-475, DOI 10.1007/BF01194647 (German). MR1545179

[78] K. Weierstrass, Abhandlungen aus der Funktionenlehre, Julius Springer, Berlin, 1886.

[79] Clifford E. Weil, On nowhere monotone functions, Proc. Amer. Math. Soc. 56 (1976), 388-389, DOI 10.2307/2041644. MR396870

[80] Z. Zahorski, Sur la première dérivée, Trans. Amer. Math. Soc. 69 (1950), 1-54, DOI 10.2307/1990595 (French). MR37338 


\section{Index}

AC, 31,33

B , 12

C, 12

CIVP, 31, 37

$\mathrm{C}^{1}, 23$

$\mathrm{C}^{1}$-norm, 5, 23

Conn, 31, 36

$\mathrm{D}_{\Phi}, 24$

D, 12, 23

$\mathcal{D}, 30$

$\Delta, 37$

$\mathrm{E}_{\Phi}, 24$

ES, 37

Ext, 31

$\mathrm{M}_{\Phi}(\mathbb{R}), 26$

$\mathrm{M}_{\Phi}^{\nearrow}(\mathbb{R}), 26$

$\mathrm{M}_{\Phi}^{\searrow}(\mathbb{R}), 26$

$\mathrm{M}_{\Phi}(\mathbb{R}), 26$

$\mathcal{M}, 12$

$\mathcal{N}, 12$

PC, 31

PR, 31, 33

$\mathcal{P}, 5,12$

SCIVP, 31

SZ, 30
$\mathcal{T}, 12$

$\mathcal{V}, 12$

ZFC, 30

$\mathcal{P}_{\mathrm{b}}, 5$

$\operatorname{cov}_{M}=\mathfrak{c}, 30$

$\mathbb{B}, 34$

$\mathcal{I}$-approximately continuous function, 30

$\rho$-ball, 26

additive function, 45

almost continuous function, 31, 33

approximately continuous function, 19, 25, 30

Baire category theorem, 5, 34

Baire class one function, 5, 24, 31, 34

Baire class two function, 31

Baire function, 30

Baire space, 24

Banach space, 5, 23

blocking set, 33

Borel function, 30

bounded variation, 4

Cantor intermediate value property, 31, 37

Cantor ternary set, 20

connectivity function, 31, 36 
continuum, 24, 25, 29

Continuum Hypothesis, 30

Darboux function, 30

Darboux property, 3

Darboux-like function, 30

dense $G_{\delta^{-} \text {-set, } 23}$

differentiable function, 12

differentiable monster, 2, 12, 19

everywhere surjective function, 37

extendable function, 31

first category, 26

generalized continuous function, 30

intermediate value property, 3,29

iterated perfect set model, 30

Jarník's extension theorem, 20

Jordan-like decomposition, 18

Lebesgue density point, 24
Lebesgue measurable function, 30

meager set, 30

nowhere-monotone, 2

P-fillings, 6

perfect road, 31, 33

perfect set, 19, 33, 37

peripherally continuous function, 31

Pompeiu derivative, 3

Pompeiu-like function, 3, 12

residual, 23

Robert's set, 42

Sierpiński-Zygmund function, 29

strong Cantor intermediate value property, 31

supremum norm, 23

typical, 23

Weierstrass's monster, 1, 19 